NOTICE: this is the author's version of a work that was accepted for publication in the journal Tectonophysics. Changes resulting from the publishing process, such as peer review, editing, corrections, structural formatting, and other quality control mechanisms may not be reflected in this document. Changes may have been made to this work since it was submitted for publication. A definitive version was subsequently published in the journal Tectonophysics, Vol.631 (2014). DOI: http://doi.org/10.1016/j.tecto.2014.04.021 


\title{
Estimation of the effective elastic thickness of the lithosphere using inverse spectral methods: the state of the art
}

Jon F. Kirby

Department of Spatial Sciences, Curtin University, GPO Box U1987, Perth, WA 6845, Australia. Tel: +61 89266 7701; fax: +61 89266 2703; email: j.kirby@curtin.edu.au.

\begin{abstract}
The effective elastic thickness $\left(T_{e}\right)$ is a geometric measure of the flexural rigidity of the lithosphere, which describes the resistance to bending under the application of applied, vertical loads. As such, it is likely that its magnitude has a major role in governing the tectonic evolution of both continental and oceanic plates. Of the several ways to estimate $T_{e}$, one has gained popularity in the 40 years since its development because it only requires gravity and topography data, both of which are now readily available and provide excellent coverage over the Earth and even the rocky planets and moons of the solar system. This method, the 'inverse spectral method', develops measures of the relationship between observed gravity and topography data in the spatial frequency (wavenumber) domain, namely the admittance and coherence. The observed measures are subsequently inverted against the predictions of thin, elastic plate models, giving estimates of $T_{e}$ and other lithospheric parameters. This article provides a review of inverse spectral methodology and the studies that have used it. It is not, however, concerned with the geological or geodynamic significance or interpretation of $T_{e}$, nor does it discuss and compare $T_{e}$ results from different methods in different provinces. Since the three main aspects of the subject are thin elastic plate flexure, spectral analysis, and inversion methods, the article broadly follows developments in these. The review also covers synthetic plate modelling, and concludes with a summary of the controversy currently surrounding inverse spectral methods, whether or not
\end{abstract}


the large $T_{e}$ values returned in cratonic regions are artefacts of the method, or genuine observations.

\section{Keywords}

Elastic thickness; Lithospheric flexure; Inverse methods; Spectral estimation.

\section{Introduction}

The Earth's tectonic plates, whether continental or oceanic, comprise the crust and uppermost mantle, and form its lithosphere. Hence, tectonic evolution and dynamics are governed primarily by the properties of the lithosphere. One of these properties is the flexural rigidity $(D)$, a measure of a material's deformability or stiffness, and whose magnitude determines the degree to which the lithosphere bends under applied, vertical loading, whether from above, below or within. While flexural rigidity can describe materials of varying rheologies, it is more commonly represented by the effective elastic thickness $\left(T_{e}\right)$, being the thickness that the plate would have for it to possess a purely elastic rheology. The relationship between the flexural rigidity and the effective elastic thickness is given by the equation:

$$
D=\frac{E T_{e}^{3}}{12\left(1-v^{2}\right)}
$$

where the elastic constants are $E$ and $v$, being Young's modulus and Poisson's ratio, respectively. As such, $T_{e}$ does not, in general, represent a depth to any boundary within the lithosphere: it is a purely geometric analogue of the integrated strength of the lithosphere (e.g., Burov and Diament, 1995; Lowry and Smith, 1995; Watts and Burov, 2003).

Of course the actual lithosphere comprises materials of many types of rheology (elastic, plastic, viscous, etc.), and the crust may or may not be decoupled from the lithospheric 
mantle (Burov and Diament, 1996). Fortunately, over loading timescales of tens of millions of years or more the lithosphere can be modelled as flexing like a thin, elastic plate, even though the actual behaviour may be anelastic (e.g., Watts, 2001; Watts and Burov, 2003; Burov and Watts, 2006; Burov, 2011; Watts, 2011). This means that $T_{e}$ provides a good measure of its flexural rigidity, and takes on an actual geological significance (e.g., Watts, 2001). As McKenzie (2003) writes, much of the tectonics of continents is likely to be controlled by the thickness of the elastic part of the lithosphere. For instance, Mouthereau et al. (2013) recently used a global $T_{e}$ model to show that the amount of shortening in collisional orogenic belts is correlated with the age of the foreland lithosphere at the time of collision. Thus strength influences the stability of continental subduction, a result also demonstrated in numerical modelling by Burov and Watts (2006). And using their own global $T_{e}$ model, Audet and Bürgmann (2011) provided evidence that deformation during supercontinent cycles is controlled by the pre-existing structure of the lithosphere, with weak zones at continental margins preserving their weakness despite orogenic recycling, and isolating strong continental interiors.

In flexural isostatic studies, the observed gravity and topography are compared against theoretical models, and several parameters of the lithosphere may be estimated. In the simplest model, a surface load flexes the plate, with the magnitude of the resulting deflection being governed by $T_{e}$. If the plate has no rigidity whatsoever $\left(T_{e}=0\right)$, the surface load will be compensated in hydrostatic equilibrium, a case referred to as "local isostasy" and described by models such as the Airy-Heiskanen or Pratt-Hayford (e.g., Watts, 2001). As the flexural rigidity increases, the load becomes additionally supported by stresses within the plate, and the plate deflects less; this being the case of "regional" or "flexural isostasy", a model first proposed by Vening Meinesz (e.g., Watts, 2001). As the lithosphere bends, so do subsurface 
interfaces, and these perturbations of internal density contrasts (with the primary, compensating density contrast usually taken at the Moho) generate a gravity anomaly. Hence, by the methods described here, comparison of the observed gravity and topography can yield $T_{e}$. With the inverse spectral method, this comparison is carried out in the spatial frequency (“wavenumber”) domain, via use of the Fourier - or very recently wavelet - transform.

The purpose of this review article is not to justify the use of $T_{e}$, however, nor to discuss its rheological or geophysical significance, nor to recount the geological interpretations of $T_{e}$ estimates obtained by the various studies that have calculated it. Instead the discussion will concern developments in one of the three most commonly-used methods by which $T_{e}$ is estimated, that of the inversion of the spectral properties of the gravity field and topography, and will not be concerned with the other two, namely forward modelling of gravity and topography in the space domain, and rheological modelling, except in brief in the Discussion. The article will focus only on the isotropic response to loading of the lithosphere, rather than on those recent studies that have investigated mechanical anisotropy. In order to explain these developments it is necessary to go into some mathematical detail of the concepts involved, so as well as being a review of inverse spectral methodology, the article should provide a brief tutorial to those unfamiliar with the subject.

The article is divided into the three main aspects of inverse flexural modelling: plate flexure models, spectral estimation, and inversion methods. First discussed is the thin, elastic plate model and its theoretical predictions, against which the observables are inverted. This is followed by an explanation of the two spectral quantities that form the observables, the admittance and coherence between gravity and topography, and the most commonly-used spectral analysis methods by which to estimate them. The inversion procedures and methods 
form the topic of the subsequent section, while the following section concerns synthetic modelling, the technique used to assess the accuracy of the inverse spectral method. Finally, the review concludes with arguments surrounding the efficacy of the method in general, and the current controversy surrounding it.

\section{Historical Overview}

The inception of inverse spectral methodology is generally attributed to Dorman and Lewis (1970) who developed (or borrowed) spectral analysis methods for the purposes of isostatic analyses, though they did not seek to estimate $T_{e}$. Dorman and Lewis (1970) suggested the use of a linear response function technique by which to relate the observed topography of the Earth $(h)$ to the gravity anomaly due to the isostatic compensation of that topography, or ‘compensation anomaly', $\Delta g_{c}$. Starting from an equation for the Newtonian gravitational potential of the compensating masses, they arrived at the formula:

$$
\Delta g_{c}=q * h
$$

where the asterisk indicates space-domain convolution in a Cartesian frame. They gave the name "isostatic response function" to the term $q$ (though used the symbol $r$ instead), but this name was later poached by Cochran (1979) to mean something rather different (Section 3.3.10). Regardless, $q$ is the (linear) response of the Earth's gravity field to a point load, and for the purposes of Dorman and Lewis (1970) contained information about the depths and densities of subsurface masses that compensate the surface topography, which they sought to extract by inverting $q$. Their first step was to write the observed Bouguer anomaly $\left(\Delta g_{B}\right)$ as the sum of the compensation anomaly and a term they called the 'geologic effect' $\left(\Delta g_{n}\right)$, thus:

$$
\Delta g_{B}=\Delta g_{c}+\Delta g_{n}
$$

The geologic effect is interesting because it provides that part of the gravity field that cannot be represented by the isostatic model (which is exhaustively given by q); in this respect it is 
the isostatic anomaly (e.g., Heiskanen and Moritz, 1967; Banks et al., 1977), and was used as such by Lewis and Dorman (1970). The importance of $\Delta g_{n}$ has only come to light in the past two decades, in that it has forced researchers to question how noise is treated in the system. Since $\Delta g_{n}$ represents the failure of the isostatic model, it has also become known as “geological noise”, "gravitational noise” or simply “noise”. The topic will be returned to in Sections 3.3.9, 4.1.4, 6.2.5 and 8.

By virtue of Eq. (2), Dorman and Lewis (1970) assumed, reasonably, that $\Delta g_{n}$ must be largely uncorrelated with the topography, and this presented a method to estimate $q$. As will be shown in Section 4.1.1, they took the Fourier transform of Eq. (3) which eventually enabled them to obtain a noise-free estimate of a frequency-domain transfer function $(Q)$, with $q$ being the inverse Fourier (or in their approach, inverse Hankel) transform of $Q$. Dorman and Lewis (1970) then showed how $q$ could be inverted for a depth-density profile describing the subsurface masses compensating the topography. In order to overcome non-uniqueness, they assumed that the compensation of a point load occurred only directly beneath the load (local isostasy), rather than it being supported by mechanical stresses spatially distributed around the load site (regional isostasy), i.e., they assumed that $T_{e}=0 \mathrm{~km}$.

The estimation of $T_{e}$ was not made in this fashion until several years later, when McKenzie and Bowin (1976) recognised that the transfer function $Q$ (they called it $Z$ ) was the “admittance” described by Munk and Cartwright (1966). McKenzie and Bowin (1976) estimated $Q$ from ship track gravity and bathymetry data, but whereas Dorman and Lewis (1970) and Lewis and Dorman (1970) had assumed local isostasy and computed and inverted q, McKenzie and Bowin (1976) made no such assumptions and did not invert $q$. Instead they used models of plate flexure to derive an analytic formula for a theoretical $Q$, which 
depended upon, amongst other things, the elastic thickness $T_{e}$ (Section 3.3). Thus by varying $T_{e}$ and the other parameters, they could find a best-fitting model to the observed $Q$ from the ship data. McKenzie and Bowin’s (1976) study heralded the start of inverse spectral methods for $T_{e}$ estimation, and the admittance method was taken up by many researchers and applied to both oceanic and continental data.

The method just described, comparing observed and theoretical admittances, had the surface topography as the only acting load. While it was used most often in oceanic settings, the few continental studies that used this approach retrieved very low values of $T_{e}$, even in regimes where it was expected to be high, such as cratons and shields. Forsyth (1985) suggested that this occurred because (1) the admittance preferentially weights regions of high topography, such as orogens, where $T_{e}$ is likely to be low; and (2) only one load (at the surface) was being modelled, whereas many geological processes can produce loads within the lithosphere. Forsyth (1985) found that if such internal loads are present in reality, but neglected in the model, then inversion of the observed admittance would yield underestimates of the true $T_{e}$. He therefore proposed that, since there are two observables (gravity and topography), flexure models could include two initial loads to be solved for. His second advance proposed the use of the coherence between gravity and topography, rather than admittance. The coherence $\left(\gamma^{2}\right)$ is essentially the square of the Pearson product-moment correlation coefficient, but computed in the wavenumber domain. But rather than comparing an observed coherence with an analytic equation for the coherence derived from a model (as had been done previously, but with the admittance), Forsyth (1985) devised a method whereby the two initial loads were recreated from the observed gravity and topography using the plate model and an assumed value of $T_{e}$. Then the coherence between the observed gravity and topography could be compared with the coherence between the initial loads, and the $T_{e}$ varied until a best-fit 
occurred. The method, now referred to as “Forsyth’s method”, “the coherence method”, or “load deconvolution”, gained widespread popularity in continental studies, and the singleload, analytic-equation, admittance method was almost abandoned for continental $T_{e}$ estimation.

Abandoned, that is, until McKenzie and Fairhead (1997) proposed its re-introduction. They were concerned that the high values of $T_{e}$ recovered by Forsyth's method in cratons were at odds with new, hypothesised models of lithospheric rheology. The ensuing argument, dubbed “jelly sandwich” vs “crème brûlée” rheology, has several fronts, with the main three being rheological modelling, maximum earthquake depth, and $T_{e}$ estimation (e.g., Burov and Watts, 2006). As mentioned earlier in this Section, it was the role of "noise" in inverse spectral methods that was to prove most contentious, but it should be stressed that this "noise" is not instrument noise or measurement errors, but that part of the gravity anomaly that is not accounted for by the flexure model.

\section{Flexural Models}

\subsection{Thin, elastic plate flexure}

When using inverse spectral methods, the lithosphere is most commonly modelled as a thin, elastic plate (in 2D, or beam in 1D) of thickness $T_{e}$, emplaced upon an inviscid substratum taken to be the asthenosphere (e.g., Watts, 2001). A thin plate is one whose deflections under loading are small compared to the thickness of the plate, and whose thickness is small compared to its lateral extent (usually taken to be infinite). As mentioned previously (Section 1), the complex multi-layered rheologies of the continental lithosphere may be modelled by an equivalent elastic plate, whose thickness $\left(T_{e}\right)$ represents the lithosphere's integrated strength (Watts and Burov, 2003). Since the thin-plate equations are rheology-independent 
(Burov et al., 1998), irrespective of whether the actual lithosphere is wholly or partly elastic, the flexural rigidity remains a measure of the deformability or stiffness of the plate (Banks et al., 1977). While the thin elastic plate model is undoubtedly idealistic - e.g., such a plate can store unlimited stress without fracture (Watts, 2001) - it has enjoyed considerable success in representing the behaviour of a loaded lithosphere.

Consider the general case of an isotropic, rectangular, thin, elastic plate of non-uniform flexural rigidity, $D(\mathbf{x})$ (where $\mathbf{x}$ is the 2D Cartesian position vector in the space domain), subject to a transverse (vertical) load, $l$, only, that causes a deflection $w$. We will not consider horizontal forces as some authors have done (e.g., Stephenson and Lambeck, 1985b), though these are mentioned in Section 3.4. If the plate rests upon an inviscid fluid then there will also be a buoyancy force, $p$, caused by the displacement of the fluid, which is simply a constant multiple of the deflection. Timoshenko and Woinowsky-Krieger (1959) give the partial differential equation describing the resulting two orthogonal bending moments $M_{x}$ and $M_{y}$, and the twisting moment, $M_{x y}$, as:

$$
\frac{\partial^{2} M_{x}}{\partial x^{2}}-2 \frac{\partial^{2} M_{x y}}{\partial x \partial y}+\frac{\partial^{2} M_{y}}{\partial y^{2}}+p=-l
$$

Here it is assumed that the deflection is small compared to the plate thickness $\left(T_{e}\right)$, the thickness is small compared to its lateral extent and radius of curvature, and the edges of the plate are free to move in the plane of the plate (so there are no horizontal shearing forces) (Timoshenko and Woinowsky-Krieger, 1959). Eq. (4) is rheology-independent, so is valid for elastic, plastic, viscous, ductile, or mixed rheology (Burov and Diament, 1995; Burov et al., 1998; Burov, 2010). Indeed, the deflection of an inelastic plate can be modelled as the deflection of some equivalent elastic plate with spatially variable $T_{e}$, but such a $T_{e}$ would only 
be valid for instantaneous loading/unloading, and may yield incorrect stresses (but correct strains) (Burov and Diament, 1995).

Rheology enters the equations when the moments are specified, and these are obtained via a depth integration of the bending stress, which is a function of local plate curvature (Burov and Diament, 1995; Burov, 2010). The twin assumptions of plane stress from linear elasticity theory (i.e., free from vertical stresses) and of small deflections then lead to a relationship between the moments and deflections, $w(\mathbf{x})$ :

$$
\begin{aligned}
& M_{x}=-D\left(\frac{\partial^{2} w}{\partial x^{2}}+v \frac{\partial^{2} w}{\partial y^{2}}\right) \\
& M_{y}=-D\left(\frac{\partial^{2} w}{\partial y^{2}}+v \frac{\partial^{2} w}{\partial x^{2}}\right)
\end{aligned}
$$

and:

$$
M_{x y}=(1-v) D \frac{\partial^{2} w}{\partial x \partial y}
$$

where Poisson's ratio ( $v$ ) is assumed to be isotropic (e.g., Timoshenko and WoinowskyKrieger, 1959; Szilard, 1974; Watts, 2001; Turcotte and Schubert, 2002). Now substituting Eqs (5), (6) and (7) into Eq. (4) we arrive at:

$$
\nabla^{2}\left(D \nabla^{2} w\right)-(1-v)\left(\frac{\partial^{2} D}{\partial x^{2}} \frac{\partial^{2} w}{\partial y^{2}}-2 \frac{\partial^{2} D}{\partial x \partial y} \frac{\partial^{2} w}{\partial x \partial y}+\frac{\partial^{2} D}{\partial y^{2}} \frac{\partial^{2} w}{\partial x^{2}}\right)+p=-l
$$

where $\nabla^{2}$ is the 2D Laplacian differential operator. At this point it is useful to note that deflections predicted by linear elastic theory are always overestimated, with the consequence that, upon inversion of the admittance, $T_{e}$ will be underestimated (Ribe, 1982). Moreover, when deflections become similar in magnitude to the $T_{e}$ value (such as large loads on a weak plate), then nonlinear elasticity effects become important and a different treatment must be performed (Ribe, 1982). 
Eq. (8) is not used directly in inverse methods for solving $T_{e}$, though it is used to generate deflections during synthetic modelling of non-uniform $T_{e}$ plates (Section 7). Instead, Eq. (8) is used indirectly to generate theoretical admittance and coherence curves, which are then compared against the observed admittance and coherence. However, we can only proceed if we assume uniform flexural rigidity, because the analytical solution requires the Fourier transform. In that case, with constant $D$, Eq. (8) becomes the biharmonic equation for uniform, isotropic flexural rigidity:

$$
D\left(\frac{\partial^{4} w}{\partial x^{4}}+2 \frac{\partial^{4} w}{\partial x^{2} \partial y^{2}}+\frac{\partial^{4} w}{\partial y^{4}}\right)+p=-l
$$

This space-domain equation is solved using the Fourier transform (e.g., Banks et al., 1977) to give the wavenumber domain equation:

$$
D k^{4} W(\mathbf{k})+P(\mathbf{k})=-L(\mathbf{k})
$$

where $\mathbf{k}$ is the $2 \mathrm{D}$ wavenumber, $k \equiv|\mathbf{k}|$, and capital letters, $W, P$ and $L$, denote the Fourier transforms of their lower-case correspondents. Hence, the term $D k^{4} W$ is the bending stress due to the plate's finite rigidity, $P$ is the buoyancy force caused by displacement of the underlying fluid, and $L$ is the applied initial load. When $D=0$, the equations merely describe the condition of Airy isostasy.

\subsection{The effect of flexure on the gravity field}

Green's equivalent layer theorem gives the gravity effect at the surface due to a subsurface density distribution that has been flexed by an amount w (e.g., Karner, 1982; Karner and Watts, 1982). In the Fourier domain the equivalent layer theorem states that, to first order, the gravity spectrum at the surface due to a subsurface layer with relief spectrum $W$, depth $z$, and density contrast $\Delta \rho$, is given by, in $2 \mathrm{D}$ : 


$$
G(\mathbf{k})=2 \pi \mathcal{G} \Delta \rho e^{-k z} W(\mathbf{k})
$$

(e.g., Karner, 1982) where $\mathcal{G}$ is the Newtonian gravitational constant. Eq. (11) is a linear approximation to the full formula, which was given by Parker (1972) (Section 6.3).

\subsection{The admittance and coherence/coherency of the standard model}

In any flexural model, Eq. (10) is used to determine the final surface topography after flexure, and Eq. (11) the corresponding gravity anomaly. These are then used to write theoretical equations for the admittance (Section 4.1), and when there are two loads acting, the coherence or coherency (Section 4.2). Though their formulae are given elsewhere, they shall also be given here for easy reference. For gravity (Bouguer or free air) anomaly spectrum $G$, and topography spectrum $H$, the admittance is found from:

$$
Q(k)=\frac{\left\langle G(\mathbf{k}) H^{*}(\mathbf{k})\right\rangle}{\left\langle H(\mathbf{k}) H^{*}(\mathbf{k})\right\rangle}
$$

where the * indicates complex conjugation, $\mathbf{k}$ is the 2D wavenumber and $k$ its modulus, and the angle brackets indicate an averaging procedure. The coherency is:

$$
\Gamma(k)=\frac{\left\langle G(\mathbf{k}) H^{*}(\mathbf{k})\right\rangle}{\left\langle G(\mathbf{k}) G^{*}(\mathbf{k})\right\rangle^{1 / 2}\left\langle H(\mathbf{k}) H^{*}(\mathbf{k})\right\rangle^{1 / 2}}
$$

while the coherence is the modulus-squared of the coherency:

$$
\gamma^{2}(k)=\frac{\left|\left\langle G(\mathbf{k}) H^{*}(\mathbf{k})\right\rangle\right|^{2}}{\left\langle G(\mathbf{k}) G^{*}(\mathbf{k})\right\rangle\left\langle H(\mathbf{k}) H^{*}(\mathbf{k})\right\rangle}
$$

Thus, by virtue of the Hermitian $G H^{*}$ term, the admittance and coherency are complex quantities, while the coherence is real-valued. 
Many models have been used over the years, with variations in the interface at which the load acts, the number of layers in the crust, continental or oceanic environments, and the number of load surfaces, of which there can only be one or two, due to there being only two observables, gravity and topography.

\subsubsection{Key developments}

The first study to derive theoretical admittance relations for flexural isostasy was by McKenzie and Bowin (1976), who used a surface loading model in the oceans, though they used a thick, rather than thin, elastic plate model. Their model was extended by Watts (1978), Cochran (1979) and Detrick and Watts (1979) to encompass a crust of several layers; these studies all considered Airy isostasy as well. On the continents, Banks et al. (1977) were the first to develop admittance equations, loading a single-layer crust at the surface, while Karner and Watts (1982) followed suit but included the effect of sediments infilling the flexural depression. Surface loading models could only explain so much, however, and problems with fitting such models to observed data drove Louden and Forsyth (1982) and McNutt (1983) to develop subsurface-only loading models, the former in the oceans, the latter in continents. As Zuber et al. (1989) wrote, shallow subsurface loads may be crustal blocks of different compositions, sedimentary basins, or igneous intrusions, while deeper loads, at the Moho for instance, may be density anomalies due to crustal underplating, lithospheric thermal anomalies, or deeper compositional variations.

A major development occurred when Forsyth (1985) combined the surface and subsurface loading regimes described by Banks et al. (1977) and McNutt (1983). The combined loading regime enabled a predicted Bouguer coherence to be computed, though Forsyth (1985) did not derive an analytic equation for this: it was done later by Ito and Taira (2000). Indeed, 
most studies that use the Bouguer coherence use the load deconvolution method which does not require analytic equations for the admittance or coherence (see Section 6.2). Since Forsyth’s advance, most continental studies have used the combined loading model, which has surface-only and internal-only loading models as end-members. In the oceans, where internal loading is thought to be rarer (e.g., Watts, 2001), surface-loading models have dominated (Section 6.1).

Refinements to Forsyth’s combined-loading model have been useful, but slight. For example, McKenzie (2003) described surface and internal loading of a two-layered crust, with internal loading occurring at the upper/lower crust interface. Given the ready availability of global crustal density/depth models such as CRUST2.0 or CRUST1.0 (Bassin et al., 2000; Laske et al., 2013), it is now much easier to refine the loading models to the regional geology (e.g., Pérez-Gussinyé and Watts, 2005; Pérez-Gussinyé et al., 2007; Kirby and Swain, 2009), improving perhaps the resulting $T_{e}$ estimates. A final model worth mentioning here is that of Banks et al. (2001), who described subsurface loading by a thin layer within the lithosphere. In contrast to the above-mentioned models, this does not require layering of the crust, and does not require knowledge of the thin layer's density, only its depth.

\subsubsection{Formulation}

Figs 1-4 show theoretical free air admittance, Bouguer coherence, free air coherence, and geoid admittance curves generated from a three-layer crust model in a continental setting (parameters given in the captions). The curves were generated using the following formulation. 
For a general model where both surface and internal loading processes are present, the final gravity anomaly and topography are simply the sum of the gravity and topography of the two loading processes (Forsyth, 1985). For reasons that will become clear later, this model is referred to as the "expressed loading” model. Although most authors express the initial loads in terms of their topographic amplitude (e.g., Banks et al., 1977; Forsyth, 1985), I follow the approach of Banks et al. (2001) and use the load itself in the formulation (where load = density contrast $\times$ gravity acceleration $\times$ load topography), though I do not use the thin layer model of these authors. I also express the equations in terms of the final gravity anomaly, rather than the final Moho topography as Forsyth (1985) did. As Kirby and Swain (2011) showed, there are computational advantages to be gained in this manner (see Section 6.2.2).

Working in the Fourier domain (constant $D$ ) and using ${ }_{T}$ to represent the surface (top) load, and ${ }_{B}$ for the internal (basal) load, the final, post-flexure gravity and topography is written in terms of the two initial loads as a matrix equation:

$$
\left(\begin{array}{l}
G \\
H
\end{array}\right)=\left(\begin{array}{ll}
\mu_{B} & \mu_{T} \\
\kappa_{B} & \kappa_{T}
\end{array}\right)\left(\begin{array}{l}
L_{B} \\
L_{T}
\end{array}\right)
$$

where the wavenumber-dependent coefficients $\mu$ and $\kappa$ are determined by the particular model (see below).

A theoretical admittance would then be found by substituting Eq. (15) into Eq. (12). When this is done, terms such as $\left\langle L_{B} L_{T}^{*}\right\rangle$ arise. Forsyth (1985) proposed that, if the two loading regimes are independent, then these cross-spectrum terms would be zero on average because they would be uncorrelated, or alternatively, have random phases (Section 6.2). Regarding auto-spectrum terms such as $\left\langle L_{B} L_{B}^{*}\right\rangle$, he introduced a new parameter, the ratio of the internal load amplitude to that of the surface load, the 'load ratio', $f$, calculated from: 


$$
f^{2}(k)=\frac{\left\langle L_{B} L_{B}^{*}\right\rangle}{\left\langle L_{T} L_{T}^{*}\right\rangle}
$$

which, in general, is a wavenumber-dependent quantity. Thus, when $f \rightarrow 0$ surface loading dominates, and when $f \rightarrow \infty$ subsurface loading dominates. Therefore, using Eq. (16) and the assumption of uncorrelated loading, the substitution of Eq. (15) into Eq. (12) gives the combined-loading theoretical admittance:

$$
Q_{t}(k)=\frac{\mu_{B} \kappa_{B} f^{2}+\mu_{T} \kappa_{T}}{\kappa_{B}^{2} f^{2}+\kappa_{T}^{2}}
$$

where all terms are functions of wavenumber modulus, $k$. The expression for the theoretical coherency is reached by substituting Eq. (15) into Eq. (13):

$$
\Gamma_{t}(k)=\frac{\mu_{B} \kappa_{B} f^{2}+\mu_{T} \kappa_{T}}{\left(\mu_{B}^{2} f^{2}+\mu_{T}^{2}\right)^{1 / 2}\left(\kappa_{B}^{2} f^{2}+\kappa_{T}^{2}\right)^{1 / 2}}
$$

The classical theoretical coherence is the square of Eq. (18). Note that the theoretical admittance, coherency and coherence as given here are all real-valued (though see Sections 3.3.8, 4.1.3, 4.2.2, 6.2.4 and 6.2.5). Surface-only loading admittances are obtained by setting $f$ $=0$ at all wavenumbers in Eq. (17); if this is done for the coherence it will be uniformly 1, showing that a coherence can only be computed when two initial loads are acting. Subsurface-only loading admittances are obtained by letting $f \rightarrow \infty$ at all wavenumbers in Eq. (17); again, if this is done for the coherence it is uniformly 1.

The system coefficients are derived from Eqs (10) and (11). In the general case consider $n$ crustal layers $(n \geq 1)$, with loading at the base of layer $j, j=[1, n]$. Let $\Delta \rho_{i}=\rho_{i+1}-\rho_{i}$ be the density contrast at the base of layer $i$, with $\rho_{n+1}=\rho_{m}$ (mantle density), and $\rho_{0}=\rho_{f}$ (the density of the overlying fluid: water or air), and let $z_{i}$ be the depth to the base of layer $i$. Let $d$ be the (positive) depth of the ocean in the marine environment, and set $d=0$ for continental 
equations. Let the parameter $A=1$ when $G$ is a free air anomaly, and 0 when $G$ is a Bouguer anomaly. It can be shown that the gravity coefficients are given by:

$$
\begin{aligned}
& \mu_{B}=\frac{2 \pi \mathcal{G}}{\Phi g}\left[-A \Delta \rho_{0} e^{-k d}+\sum_{i=1}^{n}\left(\delta_{i j} \Phi-\Delta \rho_{i}\right) e^{-k z_{i}}\right] \\
& \mu_{T}=\frac{2 \pi \mathcal{G}}{\Phi g}\left[A\left(\Phi-\Delta \rho_{0}\right) e^{-k d}-\sum_{i=1}^{n} \Delta \rho_{i} e^{-k z_{i}}\right]
\end{aligned}
$$

where $\delta_{i j}$ is the Kronecker delta, $\mathcal{G}$ is the Newtonian gravitational constant, $g$ is the gravity

acceleration, and where:

$$
\Phi \equiv \frac{D k^{4}}{g}+\Delta \rho_{m f}
$$

The topography coefficients are:

$$
\begin{aligned}
& \kappa_{B}=\frac{-1}{\Phi g} \\
& \kappa_{T}=\frac{1}{\Delta \rho_{0} g}-\frac{1}{\Phi g}
\end{aligned}
$$

for any number of crustal layers. The theoretical admittance for local isostatic compensation models such as Airy isostasy is obtained by setting $D=0$ in Eq. (20); the coherence is unity at all wavenumbers when $D=0$. To satisfy themselves of the validity of the above equations, readers are encouraged to consult McKenzie and Bowin (1976), Banks et al. (1977), Watts (1978), Louden and Forsyth (1982), McNutt (1983), Forsyth (1985), Lowry and Smith (1994), Ito and Taira (2000), Banks et al. (2001), Watts (2001), McKenzie (2003), Kirby and Swain (2009, 2011), and Simons and Olhede (2013).

\subsubsection{Free air admittance}

As shown in Fig. 1, free air admittance functions tend to zero at long wavelengths. This reflects a local compensation mechanism such as Airy isostasy where the gravity effect of the topography is balanced by that of the displaced compensating layer (e.g., the lithospheric 
mantle), and free air anomalies are, on average, zero. At short wavelengths the curves asymptote to a value dependent on the density of the uppermost crustal layer $\left(\rho_{1}\right)$, which is the reason why such densities have often been estimated by inversion of the admittance (Section 6.1). The theoretical Bouguer admittance is simply related to the free air admittance by $Q_{B}=Q_{F}-2 \pi \mathcal{G} \rho_{1}$ (Section 4.1.2): it asymptotes to zero at short wavelengths because the gravity effect of the (uncompensated or supported) topographic masses has been removed.

Fig. 1 shows that the free air (and Bouguer) admittance is highly variable for different combinations of $T_{e}, f$ and $z_{l}$ (depth to internal load). As $T_{e}$ increases (for constant $f$ and $z_{l}$ ), the admittance transition wavelength shifts to longer wavelengths and departs considerably from Airy isostasy. If any internal loads are present, their effect upon the admittance is amplified as the load proportion increases, so that in certain circumstances the admittance becomes negative. And as the internal load becomes more shallow, the admittance transition becomes steeper, almost mimicking the effect of increasing $f$.

\subsubsection{Bouguer coherence}

The characteristic shape of the Bouguer coherence curve (Fig. 2) is due to the lithosphere being unable to support very long wavelength loads without flexing, generating a Bouguer anomaly as the higher-density mantle is displaced. There is hence a strong coherence between Bouguer anomaly and topography $(\approx 1)$ at these wavelengths, and local (e.g., Airy) isostasy is approached. For short wavelength loads, however, the lithosphere appears rigid, does not flex, and no Bouguer anomaly is produced: there is thus little correlation between Bouguer anomaly and topography, and the Bouguer coherence approaches zero. The transition (or 'rollover') wavelength, at which the Bouguer coherence curve changes rapidly from 1 to 0 gives an indication as to which wavelengths of load are compensated in hydrostatic 
equilibrium $\left(\gamma_{B}^{2} \rightarrow 1\right)$, are supported by the strength of the plate $\left(\gamma_{B}^{2} \rightarrow 0\right)$, or are partially compensated/supported $\left(\gamma_{B}^{2} \approx 0.5\right.$ ) (e.g., Zuber et al., 1989). The rollover occurs at longer wavelengths for stronger plates, and at shorter wavelengths for weaker plates, as shown in Fig. 2. Simons and Olhede (2013) have provided an analytic equation for the calculation of the rollover wavelength. Importantly, the rollover wavelength is strongly dependent upon the magnitude of $T_{e}$ in the region, and only weakly upon other parameters (such as $f$ and internal load depth), which is why this method has enjoyed considerable use.

\subsubsection{Free air coherence}

The free air coherence, however, shows as much variability as the admittance, if not more (Fig. 3). The effect of increasing $T_{e}$ serves to destroy the coherence, as does the effect of decreasing the internal load depth, and increasing $f$, because subsurface loads produce a gravity effect that becomes less correlated with the topography the more rigid the plate, the closer those loads are to the surface, or the larger the load amplitude. This high parameter dependence makes the free air coherence much less suited to $T_{e}$ estimation than the Bouguer coherence. Of course there are other factors that can lower coherence (Bouguer or free air), such as noise in the data or model. The effect of model noise is discussed in Sections 3.3.9 and 6.2.5.

\subsubsection{Geoid admittance}

As some authors have computed the admittance between geoid and topography (Section 4.1.2), a theoretical geoid admittance is readily obtained (in the planar case) by dividing the free air gravity coefficients $(A=1)$ in Eq. (19) by $g k$, where $g$ is gravity acceleration and $k$ wavenumber (e.g., Chapman, 1979). There is no danger of a singularity at the zero 
wavenumber due to the presence of the exponential terms, as Fig. 4 shows. The variation of the geoid admittance with the parameters is similar to that of the free air admittance.

Chapman (1979) and McAdoo and Sandwell (1989) also provide an equation for the vertical deflection admittance.

\subsubsection{Oceanic versions}

Figs 1-4 show the admittance/coherence curves for land-loading. At sea, the Bouguer coherence curves change very little, the change in the free air coherence curves is more pronounced, while the free air admittance curves change considerably at the short wavelengths, becoming damped by the $\exp (-k d)$ term in Eq. (19). The geoid admittance is damped across all wavelengths. Performing inversions over regions containing both continent and ocean becomes problematic because the coastline produces a discontinuity in the $\mu$ and $\kappa$ coefficients due to the different fluid densities (air or water). Strategies to deal with this during inversion are discussed in Section 6.2.7.

\subsubsection{Load correlation in the expressed loading model}

As mentioned in Section 3.3.2, Forsyth (1985) developed the predicted Bouguer coherence model assuming that the surface and internal loading processes were independent or uncorrelated (the assumption that terms such as $\left\langle L_{B} L_{T}^{*}\right\rangle$ average to zero because they have random phases). This condition might seem unrealistic in certain scenarios (e.g., massive volcanism), so a handful of authors have attempted to model the case of correlated loads. Jin et al. (1994), Canales and Dañobeitia (1998), McGovern et al. (2002), Belleguic et al. (2005), Wieczorek (2007), Crosby (2007) all consider cases where the initial loads are correlated to 
some degree, with most providing plots of correlated-loads admittance or coherence without providing explicit equations.

Kirby and Swain (2009) also investigated initial load correlation, and with certain assumptions about the loads they developed theoretical equations similar to Eqs (17) and (18) but with the inclusion of a term that made the theoretical Bouguer coherency become positive at mid to short wavelengths (when it should always remain negative with the uncorrelated loads of the standard model). When squared to give the coherence, this would then exhibit two rollovers resulting in either over- or underestimation of $T_{e}$. This phenomenon has been observed in nature and has proved to be a useful diagnostic for load correlation (Section 6.2.6).

The same theory that predicted positive (real) Bouguer coherencies also predicted the existence of imaginary terms to the coherency and admittance (Kirby and Swain, 2009). These terms were zero when the initial loads were perfectly in phase, growing to maximal values for random-phase loads in contradiction to Forsyth’s (1985) derivation which returned a real-valued admittance (see below). The idea was not new (Section 4.1.3), for Stephenson and Beaumont (1980) and Forsyth (1985) had also written about the complex nature of the admittance: when two processes are acting (e.g., surface loading and mantle convection, or surface and internal loading) and in the absence of any other signal such as noise, while the individual admittances for each process will be real functions, the combined admittances will be, in general, complex. The admittance is the transfer function that predicts gravity from topography, as given by the Fourier transform of Eq. (2):

$$
G_{c}=Q H
$$


where $G_{c}$ is the transform of the compensation anomaly. If only one loading process acts, either surface or internal, and there are no extra signals ('noise'), then $Q$ is real-valued and, from Eq. (22), $G_{c}$ will be in phase with $H$ at all wavenumbers. But if two or more processes are combined, then, as Stephenson and Beaumont (1980) and Forsyth (1985) noted, the combined $Q$ will be complex and, in general, $G_{c}$ and $H$ will not be in phase.

However, Stephenson and Beaumont (1980) found that the imaginary component of the observed admittance over the Canadian Shield was very small and ignored it, while Forsyth (1985) was more zealous in his derivation than were Kirby and Swain (2009), and his condition of uncorrelated, random-phase loading forced the imaginary terms in his predicted admittance to zero (see Section 6.2.4). And when Kirby and Swain (2009) relaxed their assumptions (above), they also found that, with actual (real or synthetic) data rather than analytic equations, the flexural models predicted vanishingly small imaginary parts to the admittance and coherency (their $Y$ term), bringing it into partial agreement with Forsyth’s (1985) model.

Thus, the standard two-load thin plate model predicts in-phase final gravity and topography at all wavenumbers, independently of the phase difference between initial loads (Kirby and Swain, 2009). And from the argument above, this implies that in the absence of 'noise' the admittance (and therefore coherency) is real-valued.

\subsubsection{Unexpressed loading}

Another, though highly controversial, correlated loads model was developed by McKenzie (2003) in which the initial loads were not only perfectly in phase $\left(0^{\circ}\right)$ at all wavelengths, but their relative amplitudes were fine-tuned such that after flexure the resultant surface 
topography was uniformly flat, with a non-zero gravity anomaly being produced. This model was called "unexpressed loading” because the loads that generate the gravity anomaly are not expressed in the topography; unlike Forsyth’s (1985) standard model, in which all loading becomes topographically expressed after flexure (hence “expressed loading”).

Because the effects of unexpressed loading cannot be accurately explained by the expressed loading model, the unexpressed loading model effectively constitutes 'model noise' when viewed from the perspective of the expressed loading model. For this reason, the introduction of this model initiated a great debate because it apparently cast doubt upon the ability of Forsyth's (1985) method to accurately estimate $T_{e}$ in regions of subdued topography. The technical details of the ensuing argument can be found in Sections 6.2.5 and 8, after inversion methods have been discussed.

\subsubsection{Isostatic response function}

One word of warning concerns the use of the term 'isostatic response function'. This term was defined in Section 2 as being the impulse response of the Earth to loading, and was used by Dorman and Lewis (1970) to describe the inverse Fourier transform of the admittance (Section 4.1.1). However, Cochran (1979) defines the ‘isostatic response function’ $(\phi)$ at sea as the wavenumber-domain function obtained from the admittance by:

$$
\phi(k)=\frac{Q(k)}{2 \pi \mathcal{G}\left(\rho-\rho_{w}\right) e^{-k d}}
$$

for seafloor density $\rho$, water density $\rho_{w}$, and ocean depth $d$. This function is therefore normalised for variations in ocean depth and seafloor density, facilitating comparison between different regions of the ocean, where both $T_{e}$ and depth are strongly related to the age of the plate (e.g., Watts, 1978; Watts and Ribe, 1984). This kind of 'isostatic response 
function' has been largely applied in oceanic settings, though Watts (2001) gives expressions for continental environments, calling it the 'flexural response function' after Walcott (1976). Finally, Stephenson and Beaumont (1980) and Forsyth (1985) use the term 'isostatic response function' and 'admittance' interchangeably.

\subsection{Other Plate Models}

Variations to the thin, elastic plate model have been developed and used in inverse spectral methods by several authors (and by many when performing space-domain modelling, though these will not be discussed here). Even the first study that inverted the admittance (McKenzie and Bowin, 1976) considered an incompressible (thick) elastic plate, a procedure that was adopted by several studies that followed them (e.g., Watts, 1978; Cochran, 1979; Detrick and Watts, 1979). Kogan and Kostoglodov (1981) went further and developed an admittance equation for a compressible elastic plate.

One variation to the elastic plate that has occasionally been used in inverse methods is the viscoelastic plate, since it can be modelled by closed analytic wavenumber-domain equations. The problem is that one must specify (or solve for) an initial flexural rigidity, a viscoelastic relaxation time constant, and an effective rigidity that depends on both the time elapsed since loading and on the size of the load; and unfortunately the equations are nonlinear in rigidity. Furthermore one can specify a Maxwell or a linear viscoelastic model. In both, the plate behaves as an elastic plate at very short timescales, but at very long timescales it decays exponentially to approach zero rigidity in the Maxwell model, or a constant non-zero rigidity in the linear model (Watts, 2001). Although Walcott (1976) provided viscoelastic equations in the wavenumber domain, he focussed on stresses and did not invert for $T_{e}$. This was left to McNutt and Parker (1978), who modelled viscoelastic relaxation of the Australian plate (see 
Section 6.1). The reader is also referred to Karner (1982) and Watts (2001) for Maxwell and linear viscoelastic admittance formulae. Elastic-perfectly plastic plate models have been used in flexural studies (e.g., Turcotte et al., 1978; McAdoo et al., 1978) but not in inverse methods, though Billen and Gurnis (2005) write that these models, while successful at modelling ocean trench bathymetry, can be adequately replaced by purely elastic models with a spatially-variable $T_{e}$.

Stephenson and Lambeck (1985a) developed theoretical admittance formulae for a Maxwell viscoelastic plate subject to a time-dependent erosion process; the usual elastic plate equations are obtained when the erosion and viscous-relaxation time constants approach infinity. Stephenson and Lambeck (1985b) went further and included the effects of horizontal, in-plane forces, $N$. The formulation is not much more involved than the conventional method since the extra term in Eq. (9) is simply $N \partial^{2} w / \partial x^{2}$ (in 1D), which is just $-N k^{2}$ after Fourier transformation (in 1D or 2D); though $N$ is equal to the product of $T_{e}$ and the in-plane stress, so a cubic equation in $T_{e}$ must be solved to obtain this parameter. The presence of the extra terms modifies the shape of the admittance curve considerably. For example, horizontal forces increase the plate flexure, though stronger plates provide more resistance to this effect. The effect of in-plane stress, however, is most likely dependent upon many other factors; while Stephenson and Lambeck (1985b) report in-plane stresses of approximately $220 \mathrm{MPa}$ for central Australia, Jin et al. (1994) found that the addition of 500 MPa of compression to a Tibetan model only slightly modified the Bouguer coherence (and thence $T_{e}$ ). Yet as Burov (2010) states, if tectonic boundary forces are ignored, $T_{e}$ could be underestimated. 
Another potential source of error comes from fractured plates. It is easy to model a broken or fractured plate in the space domain, and this technique has been used in many forward modelling studies (e.g., Karner and Watts, 1983). However, broken plates cannot be modelled by Fourier domain equations because to do so requires a specific location for the point or line of fracture, and Fourier transforms are functions of wavenumber, not position (e.g., Karner and Watts, 1983; Crosby, 2007). As Karner and Watts (1983) and McNutt et al. (1988) state, if a continuous plate model is used to represent a broken plate, then $T_{e}$ will be underestimated. However, one would expect the error not to be too large, since the broken plate model assumes zero-stress at the fault. Even lithospheric-scale faults have some nonzero coefficient of friction that guarantees stress continuity on geological timescales, and $T_{e}$ is insensitive to reasonable ranges of the coefficient of friction (Lowry and Smith, 1995).

Finally, since spherical analyses are gaining popularity on the Earth, Turcotte et al. (1981), Lowry and Zhong (2003), Belleguic et al. (2005) and Audet (2014) provide the partial differential equation for the flexure of a thin, elastic shell of uniform flexural rigidity, while Beuthe (2008) gives the equation for a shell with spatially-variable flexural rigidity. Because a shell is a closed surface, and not of infinite extent like a plate, its differential equation contains a term for membrane stresses that does not occur in the plate flexure equations; these stresses provide additional support to long wavelength loads (Audet, 2014). Importantly, Audet (2014) also gives theoretical equations for the admittance and coherency predicted by thin shell flexure.

\section{Observed Admittance and Coherence}


The admittance and coherence are the fundamental 'observables' that are computed from gravity and topography data. These are then inverted against theoretical predictions from thin plate models to obtain $T_{e}$ estimates.

\subsection{Admittance}

\subsubsection{Definition}

As introduced in Section 2, the admittance is a wavenumber-domain transfer function that enables gravity to be predicted from topography. In this respect it is a linear filter containing terms that describe geological properties of the Earth. As illustrated by Eq. (2), the gravity effect of the isostatic compensation of topography is a convolution between that topography (h) and a linear filter ( $q$, the ‘isostatic response function' of Dorman and Lewis, 1970). The total, observed Bouguer anomaly $\left(\Delta g_{B}\right)$ is then the sum of this compensative effect and a signal that does not correlate with topography (the 'geologic effect', $\Delta g_{n}$ ), as shown by Eq. (3). Thus:

$$
\Delta g_{B}(\mathbf{x})=q(\mathbf{x}) * h(\mathbf{x})+\Delta g_{n}(\mathbf{x})
$$

where $\mathbf{x}$ is the $2 \mathrm{D}$ Cartesian position vector in the space domain, and the asterisk indicates convolution. Note that the free air anomaly can be used in place of the Bouguer anomaly in Eq. (24); in that case $q$ will be a free air, rather than Bouguer, response function - see Section 4.1.2. Also note that given the abundance of gravity and topography data nowadays, most studies use 2D data grids of $\Delta g_{B}$ and $h$, so the following formulation will be presented for 2D data. Data dimensionality will be addressed in Section 4.3.

The admittance, $Q$, is the Fourier transform of the response function $q$. Taking the Fourier transform of Eq. (24) gives, by the convolution theorem (e.g., Bracewell, 1986):

$$
G(\mathbf{k})=Q(\mathbf{k}) H(\mathbf{k})+N(\mathbf{k})
$$


where capital letters denote the Fourier transforms of the space-domain functions, and $\mathbf{k}$ is the 2D wavenumber, usually computed in rad $\mathrm{km}^{-1}$. The admittance is then found by multiplying Eq. (25) by the complex conjugate of the topography, $H^{*}$, giving:

$$
G(\mathbf{k}) H^{*}(\mathbf{k})=Q(\mathbf{k}) H(\mathbf{k}) H^{*}(\mathbf{k})+N(\mathbf{k}) H^{*}(\mathbf{k})
$$

Since the geologic effect is defined that part of the gravity field that cannot be predicted from the topography, it should be, on average, uncorrelated with the topography (e.g., Dorman and Lewis, 1970; McKenzie and Bowin, 1976; Banks et al., 1977). Hence if the terms in Eq. (26) are averaged according to some scheme, then the last term should vanish:

$$
\left\langle G(\mathbf{k}) H^{*}(\mathbf{k})\right\rangle=\left\langle Q(\mathbf{k}) H(\mathbf{k}) H^{*}(\mathbf{k})\right\rangle
$$

where the angle brackets denote the averaging process (discussed in Sections 4.3 and 5), and by definition $\left\langle N H^{*}\right\rangle=0$. If the averaging is isotropic, the admittance becomes a function of wavenumber modulus $(k \equiv|\mathbf{k}|)$ only, and Eq. (27) becomes:

$$
Q(k)=\frac{\left\langle G(\mathbf{k}) H^{*}(\mathbf{k})\right\rangle}{\left\langle H(\mathbf{k}) H^{*}(\mathbf{k})\right\rangle}
$$

(e.g., Munk and Cartwright, 1966). It should be noted that some studies have used variations of Eq. (28) to determine the admittance. Dorman and Lewis (1970) and Lewis and Dorman (1970), for example, performed a regression of the complex variables which they said avoids over-weighting regions with low topographic power. McNutt (1983) and McKenzie and Fairhead (1997) have also experimented with variations on Eq. (28), interchanging the $G$ and $H$ terms in different fashions.

Note that when the admittance is calculated from observed data, it may also include a contribution from non-isostatic dynamic forces acting on the lithosphere that also affect the 
topography and gravity field, such as mantle convection or glacial isostatic adjustment (e.g., Stephenson and Beaumont, 1980; McKenzie, 2010) - see Section 4.1.4.

\subsubsection{Gravity anomaly types}

While Dorman and Lewis (1970) used the Bouguer anomaly as $\Delta g$ when computing the admittance, numerous authors (e.g., Lewis and Dorman, 1970; McKenzie and Bowin, 1976; Watts, 1978; McKenzie and Fairhead, 1997) have used, or also used, the free air anomaly. The difference between these two gravity anomalies is of course just the Bouguer plate (in a Cartesian basis) correction plus the terrain correction: $\Delta g_{\mathrm{B}}-\Delta g_{\mathrm{F}}=-2 \pi \mathcal{G} \rho h+T$, where $T$ is the terrain correction, $\rho$ is the Bouguer reduction density, and $\mathcal{G}$ is the Newtonian gravitational constant. Therefore the difference between the Bouguer and free air admittances should be, in theory, constant: $Q_{B}-Q_{F}=-2 \pi \mathcal{G} \rho$, if the terrain correction is neglected (Lewis and Dorman, 1970; Banks et al., 2001). In practice this might not be so, arising not only from the way the two anomalies are computed, but also from differences between their spectra. Considering the first of these points, free air anomalies could be computed using the spiritlevelled spot height of the gravity observation, while possibly the Bouguer plate correction, and certainly the terrain correction, could be obtained from a digital elevation model and applied later. Banks et al. (2001) also commented on an incorrect choice of reduction density for the local geology causing spurious correlation with the topography, thus modifying the Bouguer admittance, though Daly et al. (2004) observed that such short-wavelength differences are unlikely to affect $T_{e}$ estimates. This view was also shared by McNutt (1983) who found that small changes in reduction density $\left(100-200 \mathrm{~kg} \cdot \mathrm{m}^{-3}\right)$ had an equal effect on both observed and theoretical admittances at medium to long wavelengths, resulting in very little alteration to estimated flexural parameters such as $T_{e}$. Considering the second, spectral, 
point, Banks et al. (2001) wrote that free air anomalies are more susceptible to aliasing (presumably during gridding) because they have higher power at short wavelengths than Bouguer anomalies due to the presence of the gravity effect of the topography, a view also shared by Pérez-Gussinyé et al. (2004). The aliasing would see power leakage from the short to long wavelengths (e.g., Percival and Walden, 1993), contaminating the long-wavelength free air admittance.

Other authors have computed and inverted the geoid admittance, on the Earth (e.g., Watts, 1979; Chapman, 1979; Sandwell and Poehls, 1980; Sandwell, 1982; Dixon et al., 1983; Cazenave and Dominh, 1984; Kogan et al., 1985; Heller and Marquart, 2002; Coblentz et al., 2011), and the rocky planets (e.g., Simons et al., 1994, 1997; Lowry and Zhong, 2003; James et al., 2013). Terrestrial geoid anomalies are readily estimated from satellite altimetry observations of the sea surface topography (e.g., Fu and Cazenave, 2001), and as with all manifestations of the gravity potential, a simple relationship can be formed between geoid and gravity anomaly in the wavenumber domain, leading to theoretical geoid admittance formulae (Section 3.3.6). The same applies to deflections of the vertical (the horizontal geoid gradient, also derived from altimeter data), with Chapman (1979) and McAdoo and Sandwell (1989) computing the deflection of the vertical admittance. Since geoid anomalies typically have higher power at long and intermediate wavelengths than gravity anomalies, Sandwell and Poehls (1980) and Coblentz et al. (2011) report that the geoid admittance is more useful for interpretation at such wavelengths because the geoid is more sensitive to deeper sources than is gravity. However the geoid signal is also dominated by mantle heterogeneities, and has low signal to noise ratio at short wavelengths.

\subsubsection{Admittance phase and complex representation}


As discussed (Section 3.3.8), the admittance is a complex function. Many early studies recognised the complex nature of the observed admittance (e.g., Dorman and Lewis, 1970; Lewis and Dorman, 1970; Stephenson and Beaumont, 1980), and used this to compute its phase (e.g., McKenzie and Bowin, 1976; Watts, 1978; Cochran, 1979; Detrick and Watts, 1979). The phase on the observed admittance $\left(\varphi_{Q}\right)$ reveals the phase relationship between the observed gravity and topography at different wavelengths:

$$
\tan \varphi_{Q}=\frac{\langle|G||H| \sin \delta\rangle}{\langle|G||H| \cos \delta\rangle}
$$

where $\delta$ is the phase difference between the gravity and topography, and will be wavenumber-dependent. These authors used this measure to check on the validity of the use of the flexural model when inverting the observed admittance (because models predicted real admittances), and to check for instrumental noise in the data (because noise would generally be out of phase with topography) (Lewis and Dorman, 1970; Ribe and Watts, 1982; Stephenson and Lambeck, 1985b).

When the phase on the admittance is zero (or $180^{\circ}$ ), the imaginary part of the admittance is zero and the ‘isostatic response function', $q=\mathrm{F}^{-1}[Q]$, will be an even and real-valued function (e.g., Bracewell, 1986). This was deemed desirable because the Earth filter was expected to be real (e.g., McKenzie and Bowin, 1976; Watts, 1978; Forsyth, 1985), especially when only one loading process was acting (Section 3.3.8). So most authors discussed, plotted and inverted the modulus of the admittance, having found (or implicitly made the assumption) that its phase was zero, at least at wavelengths of interest (e.g., Ribe and Watts, 1982; Forsyth, 1985). However, a real-valued $Q$ is not the only condition for a real-valued $q$ : if $Q$ is Hermitian then the Earth filter $q$ will still be real, but asymmetric (e.g., Bracewell, 1986; Kirby and Swain, 2004, 2009). And as will be discussed in Section 4.3.1, certain 
averaging procedures will always return a zero imaginary part even if it is present, while others will not. This means that care must be taken when inverting the observed admittance because, as discussed in Section 3.3.8, if conventional models of thin plate flexure are used, then only the real part should be inverted since such models predict a real-valued admittance (e.g., Lewis and Dorman, 1970; Kirby and Swain, 2004, 2009).

\subsubsection{The geologic effect, isostatic anomalies and model noise}

As written in Section 2, the geologic effect, $\Delta g_{n}$, is often thought of as the isostatic anomaly, i.e., that part of the gravity field not predicted by the flexural-isostatic model. Lewis and Dorman (1970) gave a means of computing the isostatic anomaly $\left(\Delta g_{I}\right)$ through:

$$
\Delta g_{I}=\Delta g_{B}-\mathrm{F}^{-1}[Q H]
$$

where $\Delta g_{B}$ is the observed Bouguer anomaly, $H$ is the Fourier transform of the observed topography, $Q$ is the Bouguer admittance, and $\mathrm{F}^{-1}$ is the inverse Fourier transform operator; hence, from Eqs (2) and (22), the term $\mathrm{F}^{-1}[Q H]$ is the compensation anomaly, $\Delta g_{c}$.

It is conceivable for $Q$ to be estimated in two ways, producing two different types of isostatic anomaly. First, as performed by Lewis and Dorman (1970), Q could be an observed admittance obtained from gravity and topography data via Eq. (28). In this case $\Delta g_{I}$ should be uncorrelated with topography, because if the averaging in Eq. (28) is performed so that the

condition $\left\langle N H^{*}\right\rangle=0$ is satisfied, then there is no geologic effect 'contaminating' the admittance, and the compensation anomaly would 'contain' the entire topographic signal. So here, the isostatic anomaly is indeed the geologic effect (by definition), but we do not (yet) know the lithospheric parameters or model to which the isostatic anomaly is referenced (McNutt, 1980). It should be appreciated, though, that $Q$ could contain a signal correlated 
with topography that has no isostatic/flexural origin, which means that the derived isostatic anomaly would not be an 'isostatic anomaly' in its original, geodetic, sense.

Second, $Q$ could be obtained from theoretical models, using Eq. (17), but here, if the wrong model values or even the wrong model for the region are used, then part of $\Delta g_{I}$ could be correlated with the topography. In this case, we know (or think we know) the model, but the derived 'isostatic anomaly’ might not be the geologic effect; such a case can easily arise if a flexural-model $Q$ is used when in reality dynamic support by mantle convection is present too.

A related issue, but one that is better discussed elsewhere (Section 6.2.5), is the 'model noise' of unexpressed loading proposed by McKenzie (2003) (Section 3.3.9), which is that part of the gravity field (1) uncorrelated with the topography, and (2) not predicted by the standard flexural model (and therefore admittance) of Forsyth (1985). In this respect, unexpressed loading satisfies the definition of both the geologic effect and the isostatic anomaly.

\subsubsection{Nonlinearity}

It is also possible that Dorman and Lewis's (1970) geologic effect, $\Delta g_{n}$, could contain signals that are errors arising from assuming a linear response in Eq. (24), when the actual response could be nonlinear (Banks et al., 1977). As McNutt (1979) wrote, the strict form of Eq. (24) is nonlinear in topography:

$$
\Delta g=\sum_{m=1}^{\infty}\left(q_{m} * h^{m}\right)
$$

However, Lewis and Dorman (1970), McNutt (1979) and Louden (1981) investigated this effect to second order, and found that, at least for the regions they studied, the inclusion of 
nonlinear topography terms in Eq. (31) gave insignificant corrections. McNutt (1979) did note though that nonlinear terms would be more appreciable in shallow seas and/or rough topography. A related topic is discussed in Section 6.3.

\subsubsection{Errors on the admittance}

As a final word, nearly all studies have computed errors on the admittance using its standard deviation formula:

$$
\sigma_{Q}(k)=|Q(k)| \sqrt{\frac{1 / \gamma^{2}(k)-1}{2 N}}
$$

(e.g., Munk and Cartwright, 1966; Bendat and Piersol, 2000) where $|Q|$ is the modulus of the admittance, $N$ is the number of independent estimates of the admittance, and $\gamma^{2}$ is the coherence, discussed in Section 4.2. Other methods for error estimation are available, however, such as jackknifing (Thomson and Chave, 1991; Kirby and Swain, 2013).

\subsection{Coherence}

\subsubsection{Definition}

In contrast to the admittance, which is a transfer function estimated through statistical methods, the other measure used in inverse flexural modelling is a purely statistical measure, the coherence between gravity and topography. The coherence is a measure of the phase relationship between two signals: at wavenumbers where the signals are uncorrelated, the phases of the cross-spectra are randomly distributed and averaging 'destroys' their coherence; when the signals are correlated, constructive interference yields a high coherence (Audet and Mareschal, 2007). Alternatively, the coherence measures the fraction of energy in the gravity field at a particular wavenumber that can be predicted from the topography using the admittance (Zuber et al., 1989; Lowry and Smith, 1994). The coherence is essentially the 
square of the Pearson product-moment correlation coefficient of statistics but computed in the frequency domain (e.g., Munk and Cartwright, 1966; Bendat and Piersol, 2000), as in:

$$
\gamma^{2}(k)=\frac{\left|\left\langle G(\mathbf{k}) H^{*}(\mathbf{k})\right\rangle\right|^{2}}{\left\langle G(\mathbf{k}) G^{*}(\mathbf{k})\right\rangle\left\langle H(\mathbf{k}) H^{*}(\mathbf{k})\right\rangle}
$$

Many authors (e.g., McKenzie and Bowin, 1976; Bechtel et al., 1987; Zuber et al., 1989) note that Eq. (33) can be positively biased by noise, and form an unbiased estimate of the coherence through:

$$
\hat{\gamma}^{2}(k)=\frac{N \gamma^{2}(k)-1}{N-1}
$$

(Munk and Cartwright, 1966), where $N$ is the number of independent Fourier coefficients in the waveband (Bechtel et al., 1987). The averaging will be discussed in Sections 4.3 and 5.

As with the admittance, either the Bouguer or free air anomaly may be employed, but the free air coherence has limited use in $T_{e}$ estimation (Section 3.3.5) and the Bouguer coherence is used exclusively. The use of the Bouguer coherence for the estimation of $T_{e}$ was first proposed by Forsyth (1985). Although the coherence had already been used in a more qualitative sense, as a check on the usefulness of data (e.g., McKenzie and Bowin, 1976; Banks and Swain, 1978; Watts, 1978), Forsyth gave it a central, quantitative role in $T_{e}$ estimation because he found that its shape was very sensitive to $T_{e}$, but (unlike the admittance) much less sensitive to other parameters such as load depths and subsurface densities (Section 3.3.4).

Although the strict definition of the coherence is as given in Eq. (33), some authors have averaged the ratio in that equation, rather than taken the ratio of the averages (e.g., Lowry and Smith, 1994; Armstrong and Watts, 2001; Pérez-Gussinyé et al., 2004, 2007). This procedure 
tends to yield short wavelength Bouguer coherences that approach a non-zero value, whereas thin plate flexure predicts a zero value (Section 3.3.4). Although these authors have proposed various reasons for this, the phenomenon has probably also occurred because these authors have averaged squared quantities, when they should be averaging $G H^{*}$ (which can be negative) and then squaring it. It should also be noted that high short wavelength Bouguer coherences can arise if an incorrect choice of Bouguer reduction density is made (Banks and Swain, 1978; Bechtel et al., 1987; Lowry and Smith, 1994; Wieczorek, 2007).

\subsubsection{Coherency}

The coherence, widely used as it is, suffers from the problem of information loss because of the squaring of the modulus of the cross-spectrum in the numerator of Eq. (33). This forces the coherence to become real and positive. Thus some authors (Lewis and Dorman, 1970; Jin et al., 1994; McGovern et al., 2002; Shin et al., 2007; Wieczorek, 2007; Kirby and Swain, 2009) have computed the coherency, $\Gamma$ :

$$
\Gamma(k)=\frac{\left\langle G(\mathbf{k}) H^{*}(\mathbf{k})\right\rangle}{\left\langle G(\mathbf{k}) G^{*}(\mathbf{k})\right\rangle^{1 / 2}\left\langle H(\mathbf{k}) H^{*}(\mathbf{k})\right\rangle^{1 / 2}}
$$

which, like the admittance, is complex (Kirby and Swain, 2009). So Kirby and Swain (2009) recommended that the square of the real coherency should be calculated and inverted, rather than the classical 'coherence’ as defined by Eq. (33). The importance of this distinction between coherence and coherency lies in the phase relationship between gravity and topography. At long wavelengths the Bouguer coherence is approximately +1 , reflecting Airy isostasy, whereas in actuality the Bouguer anomaly and topography are in anti-phase at these wavelengths and the coherency between them ought to be -1 . Furthermore, as Jin et al. (1994), Shin et al. (2007), and Kirby and Swain (2009) have shown, it can sometimes happen 
that the coherency becomes positive; the coherence would not distinguish between these two loading regimes, whereas the coherency does (Sections 3.3.8 and 6.2.6).

\subsubsection{Errors on the coherence}

Finally, it is important to provide errors on the coherence. Bendat and Piersol (2000) provide the following formula:

$$
\sigma_{\gamma^{2}}(k)=\left(1-\gamma^{2}(k)\right) \sqrt{\frac{2 \gamma^{2}(k)}{N}}
$$

where $N$ is the number of independent estimates of the coherence (e.g., Bechtel et al., 1987;

Zuber et al., 1989). As with the admittance, methods such as jackknifing have also been used.

\section{3 $1 \mathrm{D}$ vs $2 \mathrm{D}$, and averaging}

The choice of method to average the auto- and cross-spectra largely depends upon the dimensionality of the data and the spectral analysis method (Section 5). Here I use 1D and 2D in their mathematical sense, i.e., describing the number of independent dimensions used to locate the data in the space domain. Thus data collected along a profile are $1 \mathrm{D}$, while gridded data are 2D. I also extend the definition to topographic features and the like: a very long fracture zone is $1 \mathrm{D}$ since its cross-sectional profile can be represented by the variation of height in just one coordinate, while a seamount requires the use of two coordinates, and so is a $2 \mathrm{D}$ feature.

\subsubsection{Averaging in 2D}

For 2D data grids, whether over land or ocean, the averaging in Eqs (28), (33) and (35) was traditionally performed over $360^{\circ}$ of azimuth, via annuli in the wavenumber domain (e.g., Dorman and Lewis, 1970; Banks et al., 1977), making the admittance and coherence 
isotropic. As Banks and Swain (1978) report, the annuli should be spaced logarithmically so that equal amounts of information are present in each annulus: this also counteracts the decreasing signal-to-noise ratio at short wavelengths. Simons et al. (2000) discuss various averaging techniques used in admittance and coherence calculation, and the concept will be revisited in Section 5.

However, such annular averaging can destroy important information about phase (and anisotropy). Consider Eq. (28), the equation for the computation of the admittance: the crossspectrum between gravity and topography $G H^{*}$, is Hermitian. As Kirby and Swain (2004) and Kirby (2005) explained, $360^{\circ}$ isotropic azimuthal averaging of a Hermitian function completely removes the imaginary part because it is an odd function. This is probably why many authors state that the observed admittance is found to be real (e.g., McKenzie and Bowin, 1976; Watts, 1978; Forsyth, 1985). Kirby and Swain (2004) and Kirby (2005) recognised that, since only orientations rather than true directions are important, the azimuthal averaging needs only be performed over $180^{\circ}$ in order to achieve isotropy, and furthermore that real and imaginary parts of the admittance (rather than the modulus) should be averaged separately. This preserves the complex nature of the admittance and coherency, with subsequent inversion of their real parts only (Kirby and Swain, 2009).

\subsubsection{The 1D case}

The admittance and coherence may also be computed from 1D data, which is the approach adopted when the data are collected along ship or satellite altimeter tracks (e.g., McKenzie and Bowin, 1976; Watts, 1978, 1979; Louden, 1981; Karner and Watts, 1982; Louden and Forsyth, 1982; Ribe and Watts, 1982; Dixon et al., 1983; Cazenave and Dominh, 1984; McAdoo and Sandwell, 1989), though occasionally on profiles collected on land (e.g., 
Stephenson and Lambeck, 1985b; Harry and Mickus, 1998; Galán and Casallas, 2010), or even for line-of-sight accelerations of planet-orbiting satellites (e.g., McKenzie and Nimmo, 1997). Eqs (28) and (33) are still used, but $k$ replaces $\mathbf{k}$, and the averaging is performed either over many tracks covering the area, or over long tracks split into multiple segments. Ideally, all tracks should cover the same geology (Watts, 1978).

However, 1D analyses present their own unique problems that can potentially lead to erroneous $T_{e}$ estimates. First, Kogan and Kostoglodov (1981) investigated the effect of a profile intersecting a strongly linear feature such as a fracture zone at an oblique angle, and found that the power in the data, and hence the admittance, is shifted to longer wavelengths. Second, Ribe (1982) and Ribe and Watts (1982) addressed the issue of using 1D profiles and admittances when investigating inherently 2D geological and topographic features such as seamounts. There is a considerable difference, they found, between theoretical admittance functions describing an isolated 2D feature and a feature with the same cross-section but infinitely extended in the perpendicular direction. Watts et al. (1988), Goodwillie and Watts (1993), Lyons et al. (2000) and Billen and Gurnis (2005) have all investigated similar dimensionality effects for fracture zones and seamounts, all finding large $T_{e}$ errors if the incorrect dimensionality is assumed.

\section{Spectral Estimation}

The equations for calculating the observed admittance and coherence contain the gravity and topography auto-spectra and the cross-spectrum. Classically these were computed using a straightforward fast Fourier transform algorithm and wavenumber averaging, in which case the notation $\left\langle G H^{*}\right\rangle$ in Eqs (28) and (33) is appropriate for computation of the crossspectrum. Nowadays, however, several methods are available for estimation of the statistical 
properties of the auto- and cross-spectra, so the notation $S_{a b}$ for the cross-spectrum between any two space-domain signals $a$ and $b$ is more appropriate. Thus the observed admittance and coherence can be rewritten as:

$$
Q_{o}=\frac{S_{g h}}{S_{h h}} \quad \gamma_{o}^{2}=\frac{\left|S_{g h}\right|^{2}}{S_{g g} S_{h h}}
$$

Spectral estimation is not straightforward, because, as Karner and Watts (1982) and Lowry and Smith (1994) have observed, the act of selecting a finite region over which to compute the spectrum has the effect of applying a data window (a boxcar in this case) to a much larger data set, which biases the spectrum. Hence in this section we discuss the methods that have been used to estimate these spectra.

\subsection{Flexural wavelength}

The window size does not only affect the estimation of the spectra. An issue arises with the size of the data area or window compared to the magnitude of the elastic thickness of the region. A strong plate with high $T_{e}$ will deform over larger spatial scales in response to loading than will a weaker plate. A parameter called the flexural wavelength $\left(\lambda_{\mathrm{F}}\right)$ is often invoked, which is the wavelength of the deformation (the flexural depression and bulge) due to a point load on a plate of given flexural rigidity $D$. Macario et al. (1995) supply the following equation:

$$
\lambda_{F}=\pi\left(\frac{4 D}{g \Delta \rho}\right)^{1 / 4}
$$

for Moho density contrast $\Delta \rho$, and gravity acceleration $g$. Swain and Kirby (2003a) simplify this to $\lambda_{\mathrm{F}} \approx 29 T_{e}^{3 / 4}$, for $T_{e}$ and $\lambda_{\mathrm{F}}$ in $\mathrm{km}$, and commonly used values of the elastic constants and densities. The flexural wavelength differs from the Bouguer coherence transition wavelength (rollover), so Simons and Olhede (2013) provide an analytic equation for this. 
The problem is that small windows might be better at isolating a tectonic province with uniform $T_{e}$, but they might not capture the flexural/transition wavelengths of loads if $T_{e}$ is large. Conversely, large windows will resolve most flexural/transition wavelengths, but run the risk of capturing several provinces of differing elastic thicknesses. The window size, therefore, is crucial.

\subsection{Periodogram}

Up until the late 1990s the auto- and cross-spectra were determined from the periodograms of the data (e.g., Percival and Walden, 1993). Here, the Fourier transforms $G(\mathbf{k})$ and $H(\mathbf{k})$ are obtained from the 2D (or 1D) fast Fourier transform, and the topography auto-spectrum, for example, computed from $H(\mathbf{k}) H^{*}(\mathbf{k})=[\operatorname{Re} H(\mathbf{k})]^{2}+[\operatorname{Im} H(\mathbf{k})]^{2}$ at each 2D wavenumber $\mathbf{k}$. In the $2 \mathrm{D}$ case the spectra are then averaged around annuli in the wavenumber domain, as described in Section 4.3.1. The now-isotropic admittance or coherence are then formed according to Eq. (37). However, as Simons et al. (2003) noted, while the periodogram is a direct spectral estimator of some space-domain signal, it is not a very accurate one.

The reason for this inaccuracy lies in the phenomenon of spectral leakage, whereby power from one harmonic leaks into adjacent harmonics, biasing their power. Leakage can arise from several sources, such as aliasing, the Gibbs phenomenon generated at the data set edges, or windowing, where the multiplication of space-domain data by a window is tantamount to convolving the signal's true spectrum with the spectrum of the window. In this latter case, the window spectrum modifies the true spectrum of the signal to produce a distorted spectrum (e.g., Simons and Olhede, 2013). 
Standard techniques to mitigate spectral leakage were applied in most studies that used the periodogram, and McNutt (1983) and Diament (1985) provide a summary of many methods and their effect upon the admittance and coherence. Such mitigation methods are applied before Fourier transformation, and include zero-padding, tapering, edge-tapering, detrending, and mirroring the data about their edges (e.g., Lewis and Dorman, 1970; McKenzie and Bowin, 1976; Banks and Swain, 1978; Watts, 1978; Detrick and Watts, 1979; McNutt, 1979; Karner and Watts, 1982; Louden and Forsyth, 1982; Ribe and Watts, 1982; Dixon et al., 1983; Bechtel et al., 1987; Zuber et al., 1989; McKenzie and Fairhead, 1997; Audet and Mareschal, 2004a).

Mirroring is probably the most widely-used of the mitigation techniques, where 1D profiles are mirrored about their ends, and 2D grids mirrored about one or both of their edges (e.g., Lewis and Dorman, 1970). However, mirroring has been found to be a large cause of spectral leakage, especially with signals having red spectra such as gravity and topography, which may bias $T_{e}$ estimates upwards (e.g., McKenzie and Fairhead, 1997; Simons et al., 2000) or downwards (Audet and Mareschal, 2004a). Artificial power is introduced into the long wavelengths, an effect compounded by the generation of discontinuities in the derivatives of the signal at the edges (e.g., Ojeda and Whitman, 2002; Swain and Kirby, 2003a). Furthermore, mirroring forces the mirrored data to be an even function, which is the equivalent of applying a cosine, rather than Fourier, transform to the data: phase information is lost and the admittance will always be real (e.g., Louden, 1981; Louden and Forsyth, 1982; Ojeda and Whitman, 2002).

Data interpolation also presents a problem (and not just to the periodogram method), with Ebinger et al. (1989) and Ebinger and Hayward (1996) proposing that it introduces incoherent 
signals into the data, which biases $T_{e}$ estimates made from them. Ribe (1982) also ventured that interpolation reduces power at short wavelengths to the extent that the admittance is unusable here. This would mean that $T_{e}$ estimation might be unaffected, but density estimation would be compromised. And concerning detrending the data, Louden and Forsyth (1982) and Banks et al. (2001) found that this introduces leakage into the longest wavelengths.

Despite these issues, the mirrored periodogram method has been applied in many $T_{e}$ estimation studies, whether for single $T_{e}$ estimates over one study area (see Watts, 2001), or for the production of maps of the spatial variation of $T_{e}$ (Table 1 ), whereby $T_{e}$ is estimated in rectangular windows of moderate size that tile the study area. But now window size presents a further problem with this method. Synthetic testing by Lowry and Smith (1994) and Macario et al. (1995) found that the smaller the data area (or window size), the greater the degree of $T_{e}$ overestimation. This particular issue led Lowry and Smith (1994) to investigate an alternative method of spectral estimation.

\subsection{Maximum entropy}

Lowry and Smith (1994) made use of maximum entropy spectral estimation (MESE) (Burg, 1975) to circumvent the problem of small window sizes. In contrast to the periodogram, MESE is a stochastic approach by which to estimate the spectrum of a signal, and is meant to reduce the effects of data windowing. It does this by extrapolating the signal's autocorrelation function (the inverse Fourier transform of the power spectrum) under the condition of it having maximum entropy. For a Gaussian process, the entropy is the integral of the logarithm of the power spectrum, and this is maximised to obtain the most random of any power spectrum which is consistent with the observed data (Burg, 1975). So whereas usual methods 
extrapolate the data outside the window (for instance by assuming they are zero or periodic), MESE extrapolates the power spectrum. This gives a much higher-resolution spectral estimate (Burg, 1975), and works well for small window sizes (Simons et al., 2000). For this reason, the maximum entropy method has been used many times to create $T_{e}$ maps (Table 1 ).

Maximum entropy is not without problems, though. Lowry and Smith (1994) note that the Burg algorithm exhibits spurious peaks in the spectrum, peak splitting and poor phase representation. It is also very computationally expensive. And application of the method to synthetic data revealed (upward) bias in the $T_{e}$ estimates (A.R. Lowry, pers. comm.). Lowry and Smith (1994) also advise against mirroring data before MESE, because mirroring introduces noise into the spectra which biases the coherence upwards.

\subsection{Multitapers}

While the maximum entropy method specialises in small-window spectral estimation, another technique, the multitaper method, deals with spectral leakage exceedingly well. Multitaper spectral estimation was developed by Thomson (1982), and most often uses tapers (windows) defined by Slepian (1978) in 1D, extended to 2D by Hanssen (1997). The method was introduced to flexural studies by McKenzie and Fairhead (1997), but formalised and further developed by Simons et al. (2000).

Tapering data prior to Fourier transformation is not new, but when the data are windowed by a single function, information at the edges is lost, and the spectrum of the taper can strongly bias the spectral estimate of the data. The multitaper method was designed to combat these effects. The data are multiplied by several tapers, the data-taper product is then Fourier transformed, separately for each taper, and the resulting power spectrum is a (weighted) 
average of the spectra determined from all tapers. Importantly, the tapers developed by Slepian (1978) (referred to as discrete prolate spheroidal sequences) have the property that they form an orthogonal set (Fig. 5). This means that the spectra from the different tapers are approximately independent, and the weighted average possesses minimal spectral leakage (e.g., McKenzie and Fairhead, 1997; Simons et al., 2000). Hence, the averaging necessary for estimation of the admittance and coherence takes place over the tapers, though a further annular-averaging procedure is employed to obtain isotropic spectra (e.g., Simons et al., 2000). Tapers based on Hermite functions have also been used (Simons et al., 2003) which are also orthogonal, but the algorithm can be executed faster because there is no need to solve an eigenvalue problem or to perform matrix diagonalisation as with Slepian tapers.

The properties (e.g., wavenumber resolution and variance) of multitapered spectra depend on the choice of two parameters. The first is the bandwidth of the set of tapers, most often normalised and termed NW. The lower this number (values of 1 or 2), the better the bandresolution; the higher (values of 5 or 7) the poorer. The second parameter is the number of tapers applied to the data (K, or $\mathrm{K}^{2}$ in $\left.2 \mathrm{D}\right)$, but this is limited by the need to avoid aliasing, and the condition $\mathrm{K} \leq 2 \mathrm{NW}-1$ must be satisfied. Using more tapers reduces the variance of the spectral estimate as $1 / \mathrm{K}$, but this comes at the cost of reducing the band-resolution (e.g., Simons et al., 2000).

The multitaper method has been used extensively in admittance and coherence inversion Kirby and Swain (2011) provide a short overview. Its main application has been to the mapping of $T_{e}$ (Table 1), but for this purpose a third parameter becomes important: the size of the moving window. Fortunately the method has been extensively tested on synthetic data, and its biases are now well known. In early tests, Simons et al. (2000) found that the short 
wavelength coherence is biased upwards as NW decreases, though this would not necessarily affect the $T_{e}$ estimate. Swain and Kirby (2003a,b) constructed 'calibration curves' for tapers of different NW, K, and window size, and found a general downward bias of the $T_{e}$ recovered from the coherence relative to the known, model $T_{e}$. They noted that the downward bias increases with decreasing window size, unless the window is more than several times the flexural wavelength, and that the bias increases with increasing NW and K, both.

Pérez-Gussinyé et al. (2004) explained this by noting that, for red spectra, higher power at lower wavenumbers leaks to higher wavenumbers, increases the coherence, and as a result $T_{e}$ is underestimated; with the multitapered admittance, the process is the same but the bias is opposite and $T_{e}$ is overestimated. The leakage occurs because, for a spectral estimate at wavenumber $k$, the finite bandwidth $(W)$ of the tapers means that this estimate contains information over the wavenumber range $k \pm W$. Since $W$ is proportional to the value of NW, and inversely proportional to the data window dimension $(L)$, or $W=\mathrm{NW} / L$ (e.g., Simons et al., 2000), it can be seen that increasing NW and/or decreasing $L$ both broaden the discussed wavenumber range and the coherence (or admittance) is altered. Pérez-Gussinyé et al. (2004) also proposed that annular averaging further biases coherence and admittance through a similar mechanism, arising from the finite width of the annuli. The effect of multitaper bias on $T_{e}$ estimates is discussed further in Section 6.2.8.

When single $T_{e}$ estimates have been required, window sizes have tended to be large, $>1000$ km (e.g., McKenzie and Fairhead, 1997; Simons et al., 2000; Swain and Kirby, 2003a,b). However, when the purpose of the study has been to map the spatial variations in $T_{e}$ (Table 1), window sizes have usually been small, 300-800 km (e.g., Simons and van der Hilst, 2002; Daly et al., 2004; Audet et al., 2007; Pérez-Gussinyé et al., 2007, 2008, 2009a,b), but not 
always (e.g., Pérez-Gussinyé et al., 2004; Audet and Mareschal, 2004b). Synthetic tests by Crosby (2007), however, recommend use of windows $>3000 \mathrm{~km}$ in size if $T_{e}$ is to be recovered with minimal error, though he was referring to $T_{e}$ estimation by analytic, rather than deconvolution, inversion (Section 6).

A significant development saw the representation of Slepian tapers on the sphere by Wieczorek and Simons (2005). This now allowed for a truly spherical geometry to be employed, whereas previously, spherically-coordinated data were represented on the plane using a map projection, for example Lambert conic conformal (Lewis and Dorman, 1970), or transverse Mercator (McNutt, 1979; Daly et al., 2004), or had the geodetic coordinates merely treated as though they were Cartesian, the "plate carrée” (e.g., McKenzie, 2003). So far the spherical multitaper method has not been applied to the Earth, but was used to estimate Martian $T_{e}$ by Belleguic et al. (2005). Another advance has seen the rectangular or circular edges of the tapers adapted to have arbitrary spatial shape, on the sphere (Simons et al., 2006), and plane (Simons and Wang, 2011). Importantly, the new tapers remain orthogonal and localised in both space and wavenumber domains. These irregularly-shaped tapers have the advantage that their shape can be tailored to any geographic region or tectonic province.

\subsection{Wavelets}

For all its improvements in minimising spectral leakage, the efficacy of the multitaper method is restricted by the size of the window, notably in two respects. First, small windows run the risk of not being able to resolve large flexural wavelengths (and hence large $T_{e}$ values) as given by Eq. (38). Second, the wavenumber resolution of the spectra is fixed by the window size $L$, as in $W=\mathrm{NW} / L$ (Section 5.4), so small windows will have a large 
bandwidth, $W$, and poor resolution in this domain. Large windows suffer less from these effects, but potentially include regimes of differing $T_{e}$.

The change in $T_{e}$ over a region, perhaps reflecting changes in geology, would change the spectral characteristics of the gravity field and topography. Such non-stationarity in data has never been dealt with well by the Fourier transform or even its moving-window variants (e.g., Addison, 2002), and was the reason for the development of the wavelet transform (Grossman and Morlet, 1984). Whatever the taper chosen, windowed Fourier transform (WFT) methods have been used to chart non-stationarity by isolating a subset of the data, multiplying this smaller area by the taper, and performing the Fourier transform on the data-taper products; then moving the window to the next location and repeating the process. Typical movingwindow sizes for the multitaper method are given in Section 5.4. However, as Fig. 6 shows, once the window size is chosen for a study, the wavenumber-domain resolution is automatically defined at all wavenumbers. And in all cases, and indeed for all spectral estimation methods, the uncertainty relation of spectral analysis must be obeyed. This states that high precision in spatial location comes at the cost of poor precision in wavenumber location, and vice versa, or $\sigma_{x} \sigma_{k} \geq 2 \pi$, where $\sigma_{x}$ and $\sigma_{k}$ are the space and wavenumber resolutions, respectively (e.g., Farge, 1992).

In contrast, the wavelet transform has variable spatial and wavenumber resolution (Fig. 6). At low wavenumbers wavelets have poor spatial, but good wavenumber precision; at high wavenumbers, they have good spatial, but poor wavenumber precision. This feature is common for all wavelets, though most have an adjustable parameter that 'fine-tunes' the trade-off while maintaining the uncertainty relation. Thus the wavelet transform can spatially separate closely spaced, high-wavenumber features, while the WFT tends to smear the 
information, either in space or wavenumber depending on the window size (Addison, 2002).

This makes the WFT more suited for analysing signals where all features appear approximately at the same scale (Kumar and Foufoula-Georgiou, 1994). The wavelet transform is not without its problems, however. As per the discussion in Section 5.4 for multitapers, when applied to data with red spectra, the finite bandwidth of wavelets can cause leakage into shorter wavelength harmonics (e.g., Daly et al., 2004; Audet and Mareschal, 2007), but this is less of an issue at the longer wavelengths due to the shape of the Heisenberg boxes (Fig. 6). However, mirroring data prior to transformation has proved to be less of a problem with wavelets, due to their localisation properties, and ensuing restriction of artefacts to the dataset edges (Stark et al., 2003; Kirby and Swain, 2008).

Importantly though, there is no need to window the data with the wavelet transform because the wavelets, which are localised in both domains, implicitly 'window' the data at scales appropriate for the features in the signal. So instead of using moving windows, the entire dataset is convolved in the space domain with a series of dilated, translated (and possibly rotated) wavelets, providing spectral estimates at every location of the data grid (e.g., Antoine et al., 2004). The wavelet transform can also be used in 'global', as opposed to its more conventional 'local', mode, where the spectrum is averaged over its spatial coordinate. In synthetic tests conducted by Kirby and Swain (2013), it was found that the global spectra from the wavelet transform had superior wavenumber resolution at long wavelengths, and lower errors, than did Slepian tapers. At short wavelengths, though, the situation reversed.

The (continuous) wavelet transform was introduced to flexural studies by Stark et al. (2003), who used tensor (2D) fourth-order derivative of Gaussian wavelets. The wavelet admittance and coherence are formed by averaging the auto- and cross-spectra over each tensor 
component. However, Gaussian wavelets do not exactly reproduce the Fourier power spectrum (Kirby, 2005), so a fairly complicated procedure was devised to ensure that the gravity and topography auto- and cross-spectra predicted by load deconvolution (Section 6.2) could be properly compared with the observed spectra, a procedure that involved integration, and the employment of a 'pseudo-coherence', a blend of observed admittance and inverse predicted admittance (Daly et al., 2004).

In contrast, the 2D Morlet wavelets (Fig. 7) introduced by Kirby and Swain (2004) do reproduce the Fourier spectrum because they are Gaussian-modulated complex exponential functions (Kirby, 2005). Because of this, Swain and Kirby (2006) were able to easily transcribe the load deconvolution equations (Sections 3.3.2 and 6.2.2) to the wavelet domain using an equivalent Fourier wavenumber, rather than having to integrate the spectra like Stark et al. (2003). Since Morlet wavelets are anisotropic, the averaging needed for the admittance and coherence occurs by obtaining auto- and cross-spectra at a series of azimuths and averaging these. If the azimuthal averaging occurs over $180^{\circ}$ in a 'fan' geometry (Kirby, 2005; Kirby and Swain, 2013), isotropic spectra are obtained. Kirby and Swain (2011) later improved the fan wavelet method by adjusting the value of the Morlet wavelet central wavenumber to improve the spatial resolution of the $T_{e}$ maps (at the expense of sacrificing wavenumber resolution).

$T_{e}$ mapping studies using wavelet methods are given in Table 1. Comparisons between wavelet and multitaper methods have been performed by Daly et al. (2004) and Audet et al. (2007) with real data, and by Pérez-Gussinyé et al. (2009a) using synthetic data. Comparisons between the periodogram, maximum entropy, multitaper and wavelet methods over the same 
geographic regions have also been performed by Audet and Mareschal (2004a,b) and Daly et al. (2004).

Notably, based on the work of McEwen et al. (2007), Audet (2011) has extended the planar fan wavelet method to a spherical basis using a stereographic projection of the Cartesian wavelets, and in Audet (2014) he then computed the spherical wavelet admittances and coherencies for the Earth, the Moon, Mars and Venus (see also Section 6.1). Unlike spherical adaptations of the multitaper method (Section 5.4), Audet (2011, 2014) have applied the approach to whole-planet analyses, rather than selecting data over a spherical cap or other region. Other spherical quasi-wavelet methods have been developed: Simons et al. (1997) used truncated spherical harmonics in a cap moved over Venus, though as Wieczorek and Simons (2005) report, the truncation generates considerable leakage and bias, and the capharmonics are not orthogonal (Dahlen and Simons, 2008); McGovern et al. (2002) applied this technique to Mars; Kido et al. (2003) employed an azimuthally-averaged Gabor function corrected for spherical geometry, though this is not strictly a wavelet transform because their spherical corrections do not preserve self-similarity at different wavelet scales (Audet, 2011).

\section{Inversion for $T_{e}$}

Once the 'observation' (admittance or coherence) has been chosen, the spectral analysis method decided upon, and the plate model selected, the final step is to invert the observations for $T_{e}$ (and perhaps other parameters). Two approaches are available. Up to 1985 the more straightforward method was used, whereby the observations were inverted against the plate model's analytic equations (the curves given in Figs 1-4), which at this time were almost exclusively surface loading (Section 6.1). While this approach is occasionally used today, after 1985 and the paper by Forsyth (1985) the more involved method was used in most 
studies (Section 6.2). This method has been referred to as 'the coherence method' or 'Forsyth’s method', but here I will use the term ‘load deconvolution' after Lowry and Smith (1994) because it can be applied to the admittance as well as the coherence.

In both methods, the model predictions are 'fit' to the observations by some method (discussed in Section 6.4) in which the unknown or desired model parameters are varied, and known or estimated parameters held fixed, typically with values constrained from independent data. For example, Young's modulus and Poisson's ratio used in Eq. (1) can be determined from seismic measurements (Banks et al., 1977) or laboratory experiments (e.g., Mueller, 2013), while seismic data can also provide depths to intracrustal layers and their densities (e.g., Lowry and Smith, 1994).

Before proceeding, it is worthwhile mentioning again that in the studies that started all this off (Dorman and Lewis, 1970, 1972; Lewis and Dorman, 1970), $T_{e}$ was not inverted for. Instead, an Airy model of isostasy was assumed and they inverted for densities and depths to compensation. It was not until Banks et al. (1977) thought that something was wrong with the compensating depths obtained by Dorman and Lewis (1972) in the USA, over $400 \mathrm{~km}$ and well into the mantle, that $T_{e}$ entered the problem (at least in continental $T_{e}$ estimation McKenzie and Bowin (1976) had earlier inverted the free air admittance for $T_{e}$ at sea). As Banks and Swain (1978) wrote, when local compensation (zero $T_{e}$ ) models are used the compensating density contrast is the deepest it could be; with regional (non-zero $T_{e}$ ) models it is always shallower.

\subsection{Analytic inversion}


As mentioned above, this method of $T_{e}$ estimation compares the observed quantities (admittance, coherence) with their predicted versions derived from the analytic equations discussed in Section 3.3. With the early, surface-only loading models $T_{e}$ was just one of a few parameters estimated in the inversion. For instance, McKenzie and Bowin (1976), Watts (1978), Cochran (1979), Detrick and Watts (1979), Kogan and Kostoglodov (1981), Karner and Watts (1982), and Louden and Forsyth (1982) all used the free air admittance to solve for the ocean depth, seafloor density and crustal thickness using Airy isostasy models at the short wavelengths of the admittance, while using flexural models to estimate $T_{e}$. McNutt (1979) also followed this procedure, but instead chose to constrain ocean depth from independent data, rather that solve for it. Other authors have chosen to solve for just crustal thickness and $T_{e}$ in the oceans (e.g., Cochran, 1980; Kogan et al., 1985), while yet others have inverted the admittance for $T_{e}$ only (e.g., Watts, 1979; Dixon et al., 1983; Watts et al., 1988; Kalnins and Watts, 2009). However, Ribe (1982) and Ribe and Watts (1982) warned that estimates of seafloor density and depth from the admittance are likely to be highly unreliable due to noise caused by interpolation contaminating its short wavelength harmonics (see also Section 5.2).

On the continents, Banks et al. (1977) developed surface loading equations and inverted the Bouguer admittance for $T_{e}$ and mantle density in the United States, though retrieved very large errors on the density. McNutt and Parker (1978) applied surface-loading elastic plate equations to the Australian Bouguer admittance to estimate $T_{e}$ only, finding very low values $(<4 \mathrm{~km})$ for this continent. Concerned by this low value, they then developed and used similar equations for a viscoelastic plate model, suggesting that relaxation had occurred over the plate’s history; Zuber et al. (1989), however, contested that the low result of McNutt and Parker (1978) arose due to the use of a surface-only loading model. Another study saw McNutt (1980) estimate crustal thickness and $T_{e}$ in North America. And although the study of 
Karner and Watts (1983) focussed upon $T_{e}$ estimation from forward modelling with a broken elastic plate at various mountain ranges, they checked these results against $T_{e}$ estimates from inversion of the admittance using a surface loading model with a continuous elastic plate and found that the estimates gained from the continuous plate were greatly underestimated. It was not until continental subsurface loading models were developed that analytic inversion became more widely used, with McNutt (1983), the originators, estimating $T_{e}$, density and compensation depth from the Bouguer admittance in California. In another study, Kogan and McNutt (1987) estimated $T_{e}$ and crustal thickness in the USSR from surface loading equations. And while Louden and Forsyth (1982) developed expressions for a subsurface loading model at sea, they did not use this to invert for $T_{e}$, rather to explain a decrease in coherence between gravity and bathymetry not predicted by the surface loading model.

The stage was set, though, for the combination of surface and subsurface loading models by Forsyth (1985), and this launched a new phase of inversion. Although Forsyth's model was used by him (and many others) to perform load deconvolution inversion (Section 6.2), some authors found it was possible to use the combined loading model to perform analytic inversions for $T_{e}$ (and other parameters). Rather than use the observed gravity and topography to estimate the initial surface and subsurface loads, and thus the 2D wavenumber-dependent load ratio, $f(\mathbf{k})$, as load deconvolution does, the analytic inversion approach uses $f$ as a fixed or free parameter to be varied or estimated, but importantly it is assumed to be wavenumberindependent. With this in mind, Kirby and Swain (2006) referred to this method as the 'uniform- $f$ ' method.

On the continents, McNutt et al. (1988) estimated $T_{e}$ and a uniform $f$ from the Bouguer admittance and coherence at thrust belts; McKenzie and Fairhead (1997) and McKenzie 
(2003) estimated $T_{e}$ and a uniform $f$ from the free air admittance in many shield regions; while Kirby and Swain (2009) produced maps of the spatial variation of $T_{e}$ and uniform- $f$ from the free air admittance over North America. In the oceans, McAdoo and Sandwell (1989) developed theoretical vertical-deflection admittance equations for combined loading to estimate $T_{e}$ and uniform- $f$ in the Pacific Ocean; Ito and Taira (2000) developed theoretical Bouguer admittance and coherence equations for combined loading to estimate $T_{e}$ and uniform- $f$ over the Ontong-Java Plateau; and Tiwari et al. (2007) did the same for the free air admittance, estimating $T_{e}$ and uniform- $f$ for the Deccan-Reunion hotspot track. PérezGussinyé et al. (2004) also developed theoretical equations for the free air admittance and Bouguer coherence, though performed analytic inversion on synthetic data only [note the error in their Bouguer coherence equation: see Audet and Mareschal (2007)].

Very recently, Audet (2014) has generated $T_{e}$ maps not only of the Earth, but also the Moon, Mars and Venus. These were computed by inversion of spherical wavelet admittances and coherencies using analytic equations from thin shell flexure models (Section 3.4).

\subsection{Load deconvolution}

\subsubsection{The rationale}

In the 1970s and early 1980s, continental $T_{e}$ estimates had been obtained by analytic inversion of the admittance using surface loading models (Sections 3.3.1 and 6.1), and the values obtained were typically low, a few tens of kilometres at most. Perplexed at such low values, especially for cratonic regions, Forsyth (1985) suggested that these were underestimates of the true $T_{e}$, and proposed two explanations. First, the admittance preferentially weights regions with high topography, and these regions tend to be younger with a weaker plate (Louden, 1981). Second, the surface-loading models used to interpret the 
admittance failed to consider the effects of loads within the plate, due to phenomena such as volcanism, underplating, metamorphism, and lithospheric thermal anomalies. Forsyth (1985) showed that the presence of significant internal loads would cause $T_{e}$ underestimates when the admittance was interpreted using a surface loading model, because internal loading generated Bouguer anomalies at shorter wavelengths than would be expected from surface loading only (Banks et al., 2001). In other words, said Forsyth, results suggesting the purely local compensation of topographic loads (very low or zero $T_{e}$ ) could also be interpreted as a very rigid plate supporting significant internal loads.

Forsyth (1985) devised a new scheme for $T_{e}$ estimation to replace the previously-used method of fitting theoretical curves to the data. He demonstrated that the new method was able to use the observed Bouguer anomaly and topography to provide estimates of the magnitudes of both the initial loads (subsurface and surface) acting on the plate, and also to predict the admittance and coherence that the plate would have under different $T_{e}$ values. Then as before, matching observed and predicted coherences gave the optimal $T_{e}$, but this time the predicted coherence was computed from the recovered initial loads, and not a theoretical equation. Forsyth's hypothesis was tested, for example, by Bechtel et al. (1987) who re-examined the findings of local isostasy in east Africa by Banks and Swain (1978): the original zero- $T_{e}$ estimate was revised to $25 \mathrm{~km}$ by the inclusion of subsurface loading in the model.

The new method was named 'load deconvolution' by Lowry and Smith (1994), but is also referred to in the literature as "Forsyth’s method”, or "the Bouguer coherence method", or just "the coherence method", somewhat confusingly because, as will be seen, load deconvolution can be used with free air anomalies or the admittance. 


\subsubsection{The method}

Forsyth (1985) realised that, given the observed gravity anomaly and topography and an assumed value for $T_{e}$, the initial surface and subsurface loads that acted on the plate could be retrieved. It is worth explaining here Forsyth’s (1985) original method, and a subtly different approach ventured by Kirby and Swain (2011).

Recall (Section 3.3.2) that Eq. (15) expresses the post-flexure gravity and topography in terms of the initial loads. Forsyth (1985) instead used initial load-topography ( $W_{I}$ and $\left.H_{I}\right)$ rather than initial load $\left(L_{B}\right.$ and $\left.L_{T}\right)$, and Moho topography $(W)$ instead of gravity anomaly $(G)$ His equivalent of Eq. (15) was:

$$
\left(\begin{array}{l}
W \\
H
\end{array}\right)=\frac{1}{\xi+r}\left(\begin{array}{cc}
r \phi & -r \\
-1 & \xi
\end{array}\right)\left(\begin{array}{l}
W_{I} \\
H_{I}
\end{array}\right)
$$

where in his one-crustal-layer model $\xi=1+\left[D k^{4} /(g \Delta \rho)\right], \phi=1+\left[D k^{4} /(g \rho)\right]$, and $r=\rho / \Delta \rho$, where $\rho$ is crustal density and $\Delta \rho$ is the density contrast at the Moho. In the method, the initial load-topographies are recovered from the observed topography and observed Moho topography by inversion of Eq. (39) with an assumed (but adjustable) value of $T_{e}$, with $W$ being derived by downward continuation of the observed Bouguer anomaly via Eq. (11). The determinant of the $2 \times 2$ matrix in Eq. (39) - or its equivalent in Eq. (15) - is zero when $D=0$, or at the zero wavenumber; these cases correspond to Airy isostasy or an infinitely large load, when the effects of surface loading cannot be distinguished from those of subsurface loading (Forsyth, 1985; Zuber et al., 1989). However, the matrix is also singular at certain combinations of density, depth to density contrasts, $T_{e}$ and wavenumber (Lowry and Zhong, 2003). 
Using the observed grids of $W$ and $H$, the initial loads are then 'deconvolved' into the final relief at both interfaces due to the two loading processes; thus $H_{T}$ and $W_{T}$ are the final surface and Moho topographies respectively from surface loading, while $H_{B}$ and $W_{B}$ are those from subsurface loading. The final surface and Moho topographies are given by the sums $H=H_{T}+$ $H_{B}$ and $W=W_{T}+W_{B}$. Forsyth (1985) then proposed that a 'predicted coherence' should be developed from the predicted Moho and surface topographies, which could then be compared with the observed Bouguer coherence; $T_{e}$ would be obtained by minimising the misfit between observed and predicted coherences using the methods discussed in Section 6.4. However, a useful predicted coherence can only be developed if one makes certain assumptions about the load regime, and Forsyth (1985) proposed that surface and subsurface loading ought to be independent processes and therefore statistically uncorrelated (as noted in Sections 3.3.2 and 3.3.8). The veracity of this assumption, and indeed Forsyth's method in general, will be discussed in Sections 6.2.5 and 8. Thus when Forsyth (1985) derived an expression for the predicted coherence between $W\left(=W_{T}+W_{B}\right)$ and $H\left(=H_{T}+H_{B}\right)$, he assumed that cross-spectral terms between surface and subsurface loading quantities, such as $\left\langle H_{B} W_{T}^{*}\right\rangle$, would average out to zero, giving the predicted Bouguer coherence as:

$$
\gamma_{p}^{2}(k)=\frac{\left|\left\langle W_{B} H_{B}^{*}+W_{T} H_{T}^{*}\right\rangle\right|^{2}}{\left\langle\left|W_{B}\right|^{2}+\left|W_{T}\right|^{2}\right\rangle\left\langle\left|H_{B}\right|^{2}+\left|H_{T}\right|^{2}\right\rangle}
$$

Indeed, $T_{e}$ can only be estimated in this way when the initial loading processes are assumed to be uncorrelated. If they are not, then the observed coherence is just reproduced exactly for all values of $T_{e}$ (Bechtel et al., 1987). So the best fitting $T_{e}$ not only fits the observed and predicted coherence but also minimises initial load correlation (Bechtel et al., 1987), while an incorrect choice of $T_{e}$ will artificially correlate the initial loads (Pérez-Gussinyé et al., 2004). 
If loads rather than load topographies, and gravity rather than Moho topography, are used, as in Section 3.3.2, then a predicted coherence formula is obtained by substituting Eq. (15) into Eq. (33). The condition of uncorrelated surface and internal loading forces cross-spectral load terms such as $\left\langle L_{B} L_{T}^{*}\right\rangle$ to zero, giving:

$$
\gamma_{p}^{2}(k)=\frac{\left\langle\mu_{B} \kappa_{B}\left|L_{B}\right|^{2}+\mu_{T} \kappa_{T}\left|L_{T}\right|^{2}\right\rangle^{2}}{\left\langle\mu_{B}^{2}\left|L_{B}\right|^{2}+\mu_{T}^{2}\left|L_{T}\right|^{2}\right\rangle\left\langle\kappa_{B}^{2}\left|L_{B}\right|^{2}+\kappa_{T}^{2}\left|L_{T}\right|^{2}\right\rangle}
$$

There is a slight advantage gained by using this, rather than Forsyth’s (1985) formulation. In using gravity rather than Moho topography, certain computational errors are avoided, in which the exponential terms in the deconvolution coefficients, Eq. (19), reach prohibitively large values during computation, biasing the predicted coherence (Kirby and Swain, 2011). Aside from this point, the two schemes are, of course, exactly equivalent.

As a last word, McGovern et al. (2002) and Lowry and Zhong (2003) used spherical load deconvolution inversion on Mars using thin elastic shell formulae.

\subsubsection{Deconvolution with the admittance}

Forsyth (1985) used the Bouguer coherence in his method, but the load deconvolution procedure can also be formulated to use the admittance (free air or Bouguer). In this case, the predicted admittance from the deconvolution method would be written, in the formulation used in this article:

$$
Q_{p}(k)=\frac{\left\langle\mu_{B} \kappa_{B}\left|L_{B}\right|^{2}+\mu_{T} \kappa_{T}\left|L_{T}\right|^{2}\right\rangle}{\left\langle\kappa_{B}^{2}\left|L_{B}\right|^{2}+\kappa_{T}^{2}\left|L_{T}\right|^{2}\right\rangle}
$$

Load deconvolution from the free air admittance was used to estimate $T_{e}$ by Pérez-Gussinyé et al. (2004) in Fennoscandia and by Pérez-Gussinyé and Watts (2005) over Europe, with 
multitapers. Using synthetic data (both uniform and variable $T_{e}$ plates) and multitapers, Pérez-Gussinyé et al. (2004) reported that the Bouguer coherence recovers $T_{e}$ values slightly better than free air admittance; they said this occurred because free air anomalies are more susceptible to spectral leakage (Section 4.1.2). However, Kirby and Swain (2008), when applying free air admittance deconvolution to synthetic data (variable $T_{e}$ plates) using wavelets, found that on average this method reproduced the $T_{e}$ variations better than deconvolution with the Bouguer coherence. The reason they gave lay in the observation by Banks et al. (2001) that for most values of $T_{e}$ and $f$, when $f>0.1$, the Bouguer/free air admittance transition is at shorter wavelengths than the Bouguer coherence rollover. This meant that the admittance transition was being resolved by wavelets with a smaller spatial scale than those resolving the longer-wavelength coherence rollover, with an associated increase in spatial resolution. However, results for individual synthetic models were much noisier than their Bouguer coherence counterparts which Kirby and Swain (2008) explained as being due to the high sensitivity of the admittance to $f$ : many different combinations of $T_{e}$ and $f$ can give similar admittance curves, allowing for rapid variation of these parameters upon inversion. The Bouguer coherence, however, is usually much more stable.

\subsubsection{The load ratio and the loads}

In contrast to analytic inversion, load deconvolution allows for the estimation of the load ratio $(f)$ without having to solve for it as an inversion parameter. Real-Earth loads will be wavenumber-dependent - as in Eq. (16) - and load deconvolution estimates them as such; analytic inversion can only treat them as being wavenumber-independent. Once the bestfitting $T_{e}$ has been found, the two loads, $L_{B}$ and $L_{T}$, can be determined by inversion of Eq. (15) which yields: 


$$
\left(\begin{array}{l}
L_{B} \\
L_{T}
\end{array}\right)=\frac{1}{\mu_{B} \kappa_{T}-\mu_{T} \kappa_{B}}\left(\begin{array}{cc}
\kappa_{T} & -\mu_{T} \\
-\kappa_{B} & \mu_{B}
\end{array}\right)\left(\begin{array}{l}
G \\
H
\end{array}\right)
$$

The loads are then substituted in Eq. (16) for the wavenumber-dependent load ratio. However, as Forsyth (1985) stated, $f$ only has significance in a limited bandwidth, which most authors take to be the wavenumbers surrounding the Bouguer coherence rollover (e.g., Stark et al., 2003; Swain and Kirby, 2006; Tassara et al., 2007). Because of this, there exists the possibility of artificial correlation between $T_{e}$ and $f$ maps because the rollover is strongly dependent upon $T_{e}$ (Tassara et al., 2007; Kirby and Swain, 2008). Maps of the spatial variation of $f$ have mostly been made using wavelet methods (Stark et al., 2003; Swain and Kirby, 2006; Tassara et al., 2007; Kirby and Swain, 2009; Jiménez-Díaz et al., 2014), but also by Nair et al. (2012) using multitapers.

Since there are only two observables, the gravity and topography, the load deconvolution method allows only for the estimation of two initial loads, as Eq. (43) suggests, and this is the path most authors have taken. However, in their studies, in Kenya and Australia, Bechtel et al. (1987) and Zuber et al. (1989), respectively, solved for three loads: at the surface, midcrust and Moho. Two of these loads (the two internal loads) cannot be independent and were obtained by downward continuation of the gravity anomaly to the two depths. Both studies, however, found that the $T_{e}$ recovered from the three-load model showed very little difference to that recovered from the conventional two-load model, primarily because the predicted Bouguer coherence is only weakly dependent upon density variations (Bechtel et al., 1987; Zuber et al., 1989; Lowry and Smith, 1994). As Stark et al. (2003) wrote, density errors do not cause significant $T_{e}$ errors because the load deconvolution procedure, via the implicit estimation of a wavenumber-dependent $f$, absorbs the density errors, and, as we have seen, the Bouguer coherence is relatively insensitive to $f$ and also to load depth (Fig. 2). This 
important point, that all density anomalies (loads) within the lithosphere are modelled by a flexural model, will be returned to in Sections 6.2.5 and 8.

This point also highlights the difference between the uniform- $f$ (analytic) inversion method and load deconvolution. Admittance and coherence profiles from real data do not often follow the smooth theoretical curves shown in Figs 1-4, being more irregular. In load deconvolution nearly all the irregularities in the observed coherence/admittance are matched in the predicted coherence/admittance by the variation of the initial loads at different wavenumbers, which manifests as a variation of $f(k)$ (Fig. 8). In contrast, in uniform- $f$ analytic inversion $f$ cannot be varied at different wavenumbers and the fit is not exact (Fig. 8). As Forsyth wrote when describing load deconvolution, "in solving for the amplitudes of the surface and subsurface loads ... we find solutions which will exactly reproduce the gravity and topographic signals, thus automatically matching the admittance” (Forsyth, 1985, $\mathrm{p} 12,629)$.

An apparent consequence of this admittance-matching, noted by Bechtel et al. (1987) and Ebinger et al. (1989), is that "the entire gravity signal can be accounted for, leaving no isostatic anomalies” (Bechtel et al., 1987, p448). However, if the isostatic anomaly is zero, then from Eq. (30), the compensation anomaly $\mathrm{F}^{-1}[Q H]$, must equal the observed gravity anomaly and therefore must even account for that part of the gravity field not correlated with the topography, the geologic effect of Dorman and Lewis (1970) (Sections 2, 4.1.1 and 4.1.4). But a real-valued admittance cannot give rise to signals that are uncorrelated with the topography when it is multiplied by the topography transform. Therefore either the admittance has a small but non-zero imaginary part (not predicted by thin plate models, 
Section 3.3.8), or there remain small but non-zero isostatic anomalies. The significance of this logic will be taken up in Section 6.2.5.

\subsubsection{Model noise}

In the standard expressed loading model used in load deconvolution the initial loads are recreated from the observed data, through Eq. (43), and thus the assumption is implicitly made that all features in the gravity/topography arise from flexure (Section 6.2.4). Hence Forsyth's (1985) observation that the predicted admittance matches the observed admittance. This means that every initial load anomaly will be expressed in the surface topography to some degree after flexure, and furthermore all post-flexure loads predicted by the thin plate model will be in phase at all wavelengths, implying a real-valued admittance (Section 3.3.8). If they are not in phase then the plate is modelled as being strong enough to resist flexure and the predicted admittance will reflect this (though it will still be real, and a small isostatic anomaly will be produced - Section 6.2.4).

However, McKenzie (2003) proposed a model in which the post-flexure loads would not be in phase, but the plate would not necessarily be strong. This was the unexpressed loading model (Section 3.3.9) where perfectly-correlated initial surface and internal loads bend the plate such that the internal loads remain to some extent and generate a gravity field, but the surface topography shows no trace of them and is flat. If this process is coupled with a conventional loading model, then the observed gravity field is the sum of the expressed and unexpressed loading-model fields, and will not be in phase with all of the topography.

McKenzie and Fairhead (1997) and McKenzie (2003) simply called this extra gravity signal "noise”, and this terminology is probably the reason why such an argument ensued. A better 
label would be "model noise" because it is not predicted by the standard loading model of Forsyth (1985) which was the most widely-used model of the time. If model noise is present, McKenzie (2003) argued, then load deconvolution should not be used because the predicted coherence and admittance, Eqs (41) and (42), are derived with the assumption that the initial (expressed) loads are statistically uncorrelated, whereas the initial unexpressed loads are perfectly correlated. Furthermore, McKenzie (2003) wrote, use of the Bouguer coherence will cause $T_{e}$ to be overestimated because the presence of the out-of-phase gravity/topography will reduce the observed Bouguer coherence - just as any noise will reduce coherence between two signals - and the rollover will migrate to longer wavelengths, indicative of a strong plate (Fig. 9a). Above all, in modelling all loading as expressed, load deconvolution will always treat large unexpressed internal loads as evidence of a strong plate because they are not in phase with the topography, as noted above, and the scenario of large expressed loads with subdued topography is modelled by high $T_{e}$.

Whether or not unexpressed loading actually exists will be addressed in Section 8, but McKenzie and Fairhead (1997) and McKenzie (2003) proposed that the observed free air coherence could be used to detect its presence. Free air anomalies, McKenzie and Fairhead (1997) suggested, are more useful for identification of uncorrelated gravity and topography than Bouguer anomalies because the Bouguer correction removes the gravitational attraction of the topography. They argued that, under the expressed loading model, the observed free air coherence should be close to unity at all wavelengths. If it were not then it revealed the existence of uncorrelated (i.e., unexpressed) final loads. Such a scenario is given in Fig. 9a,b, where the noise has reduced both Bouguer and free air coherences from their theoretical values. However, their argument (of low free air coherence revealing unexpressed loads) presupposes that the effect of expressed subsurface loading is minimal (i.e., low load ratio), 
and as Fig. 3 shows, there are many other reasons why the observed free air coherence can be low (high $T_{e}$; shallow (expressed) internal loads; high (expressed) load ratio). McKenzie (2003) later ventured a method to detect unexpressed loading based on the theoretical free air coherence, but this of course requires an input $T_{e}$ value and so is model-dependent.

Kirby and Swain (2009) devised an alternative test for uncorrelated, model noise. They reasoned that, since thin plate flexure models predict a real-valued admittance (and therefore coherency also), then if the imaginary part of the observed admittance/coherency exhibits significantly non-zero values, these must arise from the presence of uncorrelated final loads (uncorrelated gravity/topography), i.e., unexpressed loads. Kirby and Swain’s (2009) test for noise was based upon the knowledge that when noise $N$ is added to signal $A$ but not $B$, the coherence between $A+N$ and $B$ is smaller than that between $A$ and $B$. They found that the harmonics of $N$ that are correlated with $B$ manifest in the real part of the coherency between $A+N$ and $B$ (reducing it), while the uncorrelated harmonics appear in the imaginary part (increasing it). Specifically, Kirby and Swain (2009) looked at the square of the imaginary component of the observed free air coherency normalised by the total coherence (which is equivalent to $\sin ^{2} \varphi$, where $\varphi$ is the phase on the observed free air admittance). They also showed that this measure was not affected by any of the other (geological) mechanisms by which the (real) free air coherence is reduced. Importantly, Kirby and Swain (2009) found that model noise would only cause problems to the Bouguer coherence method of $T_{e}$ estimation if it occurred at wavelengths just shorter than the wavelength of the coherence rollover; if it occurred at other wavelengths it would not destroy coherence at the rollover and thus not bias $T_{e}$. 
Nevertheless, instead of the Bouguer coherence, McKenzie and Fairhead (1997) proposed that the free air admittance should be used to estimate $T_{e}$, but using analytic inversion instead of load deconvolution. The admittance should be used, they said, because it is less sensitive to uncorrelated noise than the coherence; such noise will bias the Bouguer coherence as described above (thus overestimating $T_{e}$ ), but will only increase the variance of the free air admittance without biasing it (thus increasing $T_{e}$ errors); such a scenario is shown in Fig. 9c. In this respect they effectively identified the model noise associated with unexpressed loading with the geologic effect of Dorman and Lewis (1970) (Sections 2, 4.1.1 and 4.1.4). As Crosby (2007) later found, it is possible to make reasonable estimates of the admittance when the free air coherence is as low as 0.1 (compare Figs 9b and 9c). However, Kirby and Swain (2009) noted that while the admittance is indeed less sensitive to uncorrelated noise, it is not immune to its effects, and if present in large amounts at certain wavelengths the noise could increase the variance of the admittance so much that analytic inversion would be fooled just as much as deconvolution inversion, yielding incorrect $T_{e}$ and load ratio estimates.

\subsubsection{Initial load correlation}

Returning to the issue of the assumption of the independence of initial loads in deconvolution methods, several authors have attempted to determine the degree of load correlation, though for expressed loading only. This was done by using Eq. (43) to find the initial loads corresponding to the best-fitting $T_{e}$ value, and then calculating their coherence or coherency. Bechtel et al. (1987) found evidence for load correlation at some wavelengths in Kenya, which they attributed either to their window capturing regions of varying $T_{e}$, or to genuinely correlated geological phenomena. They then went on to interpret the retrieved initial loads, as did Zuber et al. (1989) who found evidence of load correlation in central Australia. Later, Macario et al. (1995) ran synthetic models in which the degree of initial load correlation 
could be varied (Section 7), finding that the deconvolution method will return increasingly underestimated $T_{e}$ values the more the loads were correlated. They also found that the Bouguer coherence rollover becomes less steep when initial loads are correlated. Such a broadening of the rollover has also been attributed to the capturing of several provinces of different $T_{e}$ within the window (Bechtel et al., 1990), making interpretation of broad rollovers somewhat complicated (Jin et al., 1994).

As mentioned in Section 3.3.8, Kirby and Swain (2009) mathematically modelled the effect of initial load correlation upon the Bouguer coherency, and found that its real part became positive, contradicting standard uncorrelated-loading models. While they did not invert the observed coherency against such a model, they set all incidences of positive observed real Bouguer coherency in North America to zero before squaring for the 'coherence' and subsequent inversion using the classical model. Pérez-Gussinyé et al. (2009b) used this model to analyse the Bouguer coherency in Africa, finding evidence for positive initial load correlation at short wavelengths in east Africa. Finally, some planetary flexural studies have assumed perfectly-correlated initial loads (McGovern et al., 2002; Belleguic et al., 2005; Wieczorek, 2007).

\subsubsection{Inversion over a coastline}

As mentioned in Section 3.3.7, the deconvolution coefficients become discontinuous at the coastline, due to the abrupt change of overlying fluid density (air or water). Two strategies have been used to deal with this. Lowry and Smith $(1994,1995)$ and Pérez-Gussinyé et al. (2004, 2007, 2008) separated oceanic and continental data into two grids, performed load deconvolution inversions separately on each using the appropriate fluid density, then recombined the land and ocean loads by simply summing them in the Fourier domain. 
However, Kirby and Swain (2008) ventured that this would generate Gibbs phenomenon edge effects because the Bouguer anomaly is not zero at the coast. The other strategy is to convert the marine bathymetry to an equivalent topography, by finding its depth if the water were to be replaced by air, and then computing the observed admittance or coherence as usual (e.g., Stark et al., 2003). Then only land-loading equations need be used in all environments during inversion. Kirby and Swain (2008) assessed the error imparted to oceanic $T_{e}$ from this, finding that when the load ratio $f<0.3 T_{e}$ is underestimated by a maximum value of $4 \mathrm{~km}$, whereas when $f>0.3 T_{e}$ is overestimated by a maximum value of $7 \mathrm{~km}$ (assuming oceanic $T_{e}$ $<40 \mathrm{~km}$ ). The equivalent topography method can also be used with analytic inversion.

\subsubsection{Window bias: deconvolution with multitapers and wavelets}

One potential source of error is particular to windowing methods, and concerns the method and the size of the area used to estimate the predicted coherence. Multitaper spectral bias due to small window sizes has already been discussed in Section 5.4, but Pérez-Gussinyé et al. (2004) also found that if the observed coherence is computed in a relatively small window $\left(<2000 \times 2000 \mathrm{~km}^{2}\right)$ and compared against a theoretical curve, as in analytic inversion, then the resulting $T_{e}$ will be biased downwards. This happened, they said, because the theoretical curves are effectively computed using the continuous Fourier transform which assumes an infinite data area, while the observed measure is computed over a finite (small) window. They showed that the observed Bouguer coherence rollover was displaced to shorter wavelengths than the analytic predictions, the more so for small windows; Crosby (2007) found a similar result for the admittance transition. So like was not being compared with like. Instead they proposed that the same spectral estimator that was used to compute the observed coherence should be used in the predicted coherence computation. For example, if the observed coherence was estimated by applying five tapers of bandwidth parameter NW $=3$ to 
the observed gravity and topography, then the same tapered gravity and topography should be used to estimate the (tapered) initial loads via Eq. (43), and thence the predicted coherence from Eq. (41). In this fashion, the multitaper bias would be common to both observed and predicted coherence, with no ensuing bias in $T_{e}$. Incidentally, this approach of using the same spectral estimator was first suggested by Lowry and Smith (1994), but with regard to the mirrored periodogram method.

A related issue concerned the size of the area in which to perform the load deconvolution (Lowry and Smith, 1994; Pérez-Gussinyé et al., 2004). Imagine one has gravity and topography data extending over a large area A, but wishes to estimate $T_{e}$ in a smaller subregion of A, called B. One would of course have to compare the observed and predicted coherences estimated only over region B. However, it is possible for the initial loads to be recreated (deconvolved) using Eq. (43) with gravity and topography data from region A; the load spectra would then be transformed to the space domain, windowed/tapered over region B, and used to form region B’s predicted coherence. From synthetic modelling, PérezGussinyé et al. (2004) found that this adjustment to the method reduced the bias and variance of $T_{e}$ estimates retrieved from synthetic data. Other authors (e.g., Swain and Kirby, 2003a,b; Pérez-Gussinyé and Watts, 2005; Pérez-Gussinyé et al., 2007) have deconvolved the loads over the smaller region.

In addition to the downward $T_{e}$ bias caused by decreasing window size and/or increasing NW (Section 5.4), synthetic modelling by Swain and Kirby (2003a,b) showed that the downward bias in $T_{e}$ is amplified as $T_{e}$ increases. This bias arises when $T_{e}$ increases and the flexural wavelength approaches and exceeds the window size and can no longer be resolved adequately (Section 5.1). This eventually limits the maximum recoverable $T_{e}$ value for a 
given window size (e.g., Pérez-Gussinyé et al., 2007). Many other authors have noted this multitaper bias (e.g., Pérez-Gussinyé et al., 2004; Audet and Mareschal, 2004a; Daly et al., 2004; Crosby, 2007), and some have used the calibration curves discussed in Section 5.4 as ‘correction curves’ by which to adjust recovered $T_{e}$ values from real data (Pérez-Gussinyé et al., 2009a). Kirby and Swain (2011) cautioned against this practice, however, since the calibration curves are constructed from synthetic uniform- $T_{e}$ plate models of large area, and may not be appropriate for narrow $T_{e}$ anomalies. Synthetic testing by Kirby and Swain (2008), using the fan wavelet method, showed that as the $T_{e}$ anomaly's size decreases, the recovered $T_{e}$ value is lowered (or raised) to the value of the surrounding region. This occurs, they said, because large $T_{e}$ values have a large flexural wavelength which requires a largescale wavelet to resolve it; such a wavelet would be convolved with a large amount of data, thus averaging much of the surrounding $T_{e}$ and reducing the anomaly's relative magnitude. This phenomenon would not be limited to wavelet analysis either. For instance the multitaper window size would need to be large to capture a large flexural wavelength, again resulting in considerable averaging. A glance at the $T_{e}$ maps of the studies in Table 1 shows exactly this: the spatial resolution within regions of high $T_{e}$ is generally much worse than that in low $T_{e}$ regions.

Fortunately for wavelet methods window size is not an issue as it is for moving-window multitaper or periodogram techniques, because the spatial variation in $T_{e}$ is achieved by convolution rather than windowing. Most studies using wavelets have investigated the $T_{e}$ variation over very large regions (see Table 1) where the use of both analytic and deconvolution inversion is acceptable (e.g., Crosby, 2007) and the two give similar results (Stark et al., 2003; Kirby and Swain, 2004, 2006, 2009; Audet and Mareschal, 2007). 
Finally, Pérez-Gussinyé et al. (2009b) have gone further, and having obtained $T_{e}$ maps over Africa from three multitaper window sizes, they have applied the correction curves to each, but then produced a single $T_{e}$ map by calculating a weighted average of the three maps. Their rationale was that small windows give a high spatial resolution when mapping $T_{e}$ but do not resolve high $T_{e}$ values, while large windows do resolve the high $T_{e}$ values, but do not provide a good spatial $T_{e}$ resolution. They reasoned that the merged product should contain both good spatial resolution and an accurate estimate of the true $T_{e}$. Such a technique has not been performed with wavelets (though it could be by varying the Morlet wavelet central wavenumber), and it remains to be seen whether the procedure of merging $T_{e}$ maps in this fashion is statistically and spectrally valid. Nevertheless, Pérez-Gussinyé et al. (2009b) have applied the technique to synthetic data, with encouraging results. The method has also been applied to China and south Asia by Chen et al. (2013).

\subsection{Nonlinear effects}

As mentioned in Section 3.2, Green's equivalent layer theorem is used to supply the gravity effect at the surface due to flexure of a subsurface interface. But it was also mentioned there that Eq. (11) is merely a first-order approximation. The full formula giving the gravity spectrum, $G$, due to flexure of a density contrast $\Delta \rho$, at depth $z$, was given by Parker (1972) as, in 2D:

$$
G(\mathbf{k})=2 \pi \mathcal{G} \Delta \rho e^{-k z} \sum_{n=1}^{\infty} \frac{k^{n-1}}{n !} \mathrm{F}\left[w^{n}(\mathbf{x})\right]
$$

where $w(\mathbf{x})$ is the space-domain relief of the subsurface interface, $\mathrm{F}$ is the forward Fourier transform, $\mathbf{k}$ is $2 \mathrm{D}$ wavenumber and $k \equiv|\mathbf{k}|$. The equation is also valid in $1 \mathrm{D}$, though Lyons et al. (2000) found that the 1D version is only valid if the anomaly under analysis has a length much greater than its width, and that profiles intersecting it are perpendicular to its length. 
However, these nonlinear effects cannot readily be incorporated into theoretical admittance or coherence equations, and some form of numerical approach must be made. Several authors have investigated the effect of including the nonlinear terms in the inversion for $T_{e}$. Watts (1978) compared gravity over seamounts using the linear Fourier approximation against space-domain line integral methods, and found only small differences on the seamount flanks, while Watts et al. (1980) argued that the inclusion of nonlinear gravity effects would not significantly alter their conclusions. Using synthetic seamount models, Karner (1982) found that the nonlinear contributions from all density contrasts would be most pronounced at regions of high topographic gradient, which implied that the higher-order terms in Eq. (44) would make their effect felt most at the shorter wavelengths. This conclusion was also reached by Ribe (1982), who further proposed that the higher-order terms would grow large when the plate deflection was of the order of $T_{e}$ - though in that case thin plate theory would no longer be appropriate. Karner (1982) also proposed that nonlinear effects would be greater when $T_{e}$ is low, since the deflections from loading would be steeper, potentially leading to $T_{e}$ overestimation upon inversion against linear models.

In another detailed study of synthetic seamounts, Lyons et al. (2000) investigated the effect of the nonlinear terms (to $7^{\text {th }}$ order) in bathymetry and Moho topography, again finding that the consequences of not including them are very minor with regard to the Moho topography, but important over the peak of the seamount when its height is of the same order as the ocean depth. So while they noted $T_{e}$ overestimation with the synthetic models, they only found a slight difference between linear and nonlinear $T_{e}$ estimates over actual seamounts in the Louisville Ridge in the Pacific. Billen and Gurnis (2005) and Watts et al. (2006) have also investigated the effects of nonlinear terms in marine settings, with the latter study observing 
that the quality and harmonic content of the gravity data can adversely influence results with higher-order terms. Finally, in studies of continental margins, Karner and Watts (1982) proposed that spikes in the observed admittance could be modelled by the inclusion of higher-order terms (up to $n=4$ ), and furthermore, could be interpreted in terms of the width of the continental slope.

\subsection{Inversion methods and parameter errors}

The criterion of 'best fit' used in the inversion methods is usually defined by minimisation of the $\mathrm{L}_{1}$ - or $\mathrm{L}_{2}$-norms. For observations $O$ (the observed admittance or coherence) and their analytic or predicted versions $P$, these norms are:

$$
\mathrm{L}_{p}=\left(\sum_{i=1}^{N}\left|\frac{O\left(k_{i}\right)-P\left(k_{i} ; \mathbf{p}\right)}{\varepsilon_{o}\left(k_{i}\right)}\right|^{p}\right)^{1 / p}
$$

(e.g., Parker and McNutt, 1980) for $N$ independent wavenumbers $k_{i}$, where $p=1$ gives the $\mathrm{L}_{1}$ norm, and $p=2$ the $\mathrm{L}_{2}$-norm. The $\mathbf{p}$ is the parameter set (e.g., $\left.T_{e}, f, z_{l}\right)$, and the difference has been weighted by the errors on the observations, $\varepsilon_{0}$. The parameters, $\mathbf{p}$, are adjusted by some method to provide the minimum misfit.

Hence it can be seen that the $\mathrm{L}_{1}$-norm is the mean absolute deviation, while the square of the $\mathrm{L}_{2}$-norm is the chi-squared statistic. As Ojeda and Whitman (2002) state, the $\mathrm{L}_{2}$-norm is more affected by gross errors (outliers) than the $\mathrm{L}_{1}$-norm, and for this reason they, and e.g., Banks et al. (1977), Banks and Swain (1978), Sandwell and Poehls (1980), McNutt (1983), Kogan and McNutt (1987), Poudjom Djomani et al. (1995, 1999), and Flück et al. (2003) chose to minimise the $\mathrm{L}_{1}$-norm. Most other authors have minimised the chi-squared statistic or its relative, the root mean square. 
Formulae used for the observation errors, $\varepsilon_{0}$, have already been given for the admittance and coherence, Eqs (32) and (36), respectively and these have been employed by numerous authors. Some authors, however, have used the jackknife method (Thomson and Chave, 1991) to estimate these errors (Swain and Kirby, 2003b; Pérez-Gussinyé et al., 2004; Swain and Kirby, 2006). Others have simply weighted the observation-prediction difference by reciprocal-wavenumber (Kirby and Swain, 2006, 2008), or not weighted it at all because, as Audet and Mareschal (2004a) write, observation errors are usually larger around the coherence rollover which is an important diagnostic for $T_{e}$.

Few authors are explicit about the method used to find the minimum misfit. Swain and Kirby (2006) and Kirby and Swain (2008) mention that they use Brent's method (e.g., Press et al., 1992) when only one parameter $\left(T_{e}\right)$ is solved for, while Swain and Kirby (2003b) and Kirby and Swain (2009) use a least squares estimation technique given by Tarantola (1987) when two or more parameters are required. Recently, Audet (2014) has employed the neighbourhood algorithm of Sambridge (1999a,b) to search the parameter space, which has the advantage of also estimating parameter errors.

Errors on the parameters have been computed in various ways, but again, many times the specific method is not mentioned. Bechtel et al. (1987), Ebinger et al. (1989), and Ebinger and Hayward (1996) state that $T_{e}$ errors were calculated by finding the coherence curves that fit the coherence rollover within the coherence error bounds. A popular technique is to saturate the parameter space, i.e., find the $\mathrm{L}_{p}$ misfits between observed and predicted quantities for all parameter values within reasonable ranges, and then select some criterion to rule out parameter estimates with $\mathrm{L}_{p}$ values above some level, shown in Fig. 10 (e.g., Lowry and Smith, 1994; McKenzie and Fairhead, 1997; Pérez-Gussinyé et al., 2004; Kirby and 
Swain, 2009). This approach can also provide information about useful window sizes (Lowry and Smith, 1994; Pérez-Gussinyé et al., 2004), in that too small windows will yield flat misfit curves/surfaces above some $T_{e}$ value related to the relationship between window size and flexural wavelength (e.g., Pérez-Gussinyé et al., 2004).

In general, most authors have found, through synthetic modelling or otherwise, that $T_{e}$ error increases with increasing absolute $T_{e}$ value (e.g., Macario et al., 1995; Crosby, 2007; PérezGussinyé et al., 2007; Kirby and Swain, 2008).

\subsection{Space-domain filters}

Although not officially an 'inverse spectral method', and only because the admittance and coherence are not used, other methods for $T_{e}$ estimation involve some form of space-domain convolution of topography with a filter, whether the filter is determined observationally, from inverse Fourier transform of the admittance, or theoretically, from plate flexure models. The convolution yields a gravity anomaly which is then compared to observed gravity data, so in this respect the method can be thought of as a hybrid of classical forward modelling and inverse spectral methods.

Such methods have been applied largely to marine data. For example Watts (1978) and Detrick and Watts (1979) used the observed admittance, $Q_{o}$, and performed the Fourierdomain operation $\Delta g=\mathrm{F}^{-1}\left[Q_{o} H\right]$ (where $\mathrm{F}^{-1}$ is the inverse Fourier transform), while Cochran (1979) performed the space domain convolution $\Delta g=\mathrm{F}^{-1}\left[Q_{o}\right] * h$. When the admittance is determined observationally, the predicted gravity anomalies are noise-free and comparison with observed gravity data yields that part of the gravity not predicted by the filter (McKenzie and Bowin, 1976; Watts, 1978). Alternatively, a range of theoretical admittances, 
$Q_{t}$, with different $T_{e}$ values can be used to find that $T_{e}$ value that gives the smallest RMS difference between observed and predicted gravity in the space domain (Watts, 1978; Detrick and Watts, 1979; Watts et al., 1988). In a variation on this, Dixon et al. (1983), Watts et al. (1985) and Goodwillie and Watts (1993) varied $T_{e}$ in the theoretical geoid admittance formula until the predicted bathymetry, found from $h=\mathrm{F}^{-1}\left[N / Q_{t}\right]$, where $N$ is the Fourier transform of the geoid height, matched the observed bathymetry. This approach, though, requires some modification of the reciprocal admittance (e.g., Watts et al., 2006). However, as Walcott (1976) and Watts (1978) noted, this form, and indeed all forms, of forward modelling emphasise amplitude rather than wavelength when selecting the best fit.

In a similar method, Braitenberg et al. (2002, 2003, 2006) took the inverse Fourier transform of a theoretical transfer function (relating load to flexure) for an assumed $T_{e}$, and convolved this in the space domain with a load. The load was obtained from the observed surface topography and from crustal density models. The convolution yielded the predicted flexure of the plate for the chosen $T_{e}$. The predicted flexure was then compared against the observed flexure, and $T_{e}$ adjusted until a best fit occurred. The observed flexure was assumed to be given by the undulations of the Moho, which could be obtained either by inversion of the gravity anomaly or from seismic data. Braitenberg et al. (2002) claimed that this approach provided a higher spatial resolution in $T_{e}$ variations, could be applied over a smaller area than traditional inverse spectral methods, and involved independent data for the Moho undulations, whereas spectral methods merely downward continued the Bouguer anomaly. The method has also been used by Jordan and Watts (2005).

\section{Synthetic Modelling}


Synthetic modelling has formed an important part of most recent inverse spectral methods studies. It has been used to assess how well a method recovers $T_{e}$ (Macario et al., 1995), the coherence (Lowry and Smith, 1994), or the admittance (Crosby, 2007), or merely to test the accuracy of a new method, whether it be maximum entropy (Lowry and Smith, 1994), multitapers (e.g., Simons et al., 2000; Swain and Kirby, 2003a; Pérez-Gussinyé et al., 2004), or wavelets (Stark et al., 2003; Kirby and Swain, 2004, 2008; Audet and Mareschal, 2007).

The most widely-used synthetic modelling procedure was developed by Macario et al. (1995) to test the $T_{e}$ recovery capabilities (and the effects of initial load correlation) of the load deconvolution method of inversion. The method starts with the creation of two grids of fractal surfaces using the spectral synthesis algorithm of Saupe (1988). Each surface has a uniformly-distributed random phase, and a Gaussian-distributed random amplitude, so that they should be uncorrelated, though Macario et al. (1995) also provided a method for varying the degree of correlation. These grids are used as the two initial loads, surface and subsurface, acting on a plate of known $T_{e}$ (and other parameters such as densities and depth to the subsurface load). Each surface is scaled to have a realistic amplitude, with the subsurface load surface multiplied by a constant chosen to represent the desired load ratio, $f$, of the model. Grids of the final gravity anomaly and surface topography after flexure are then found by computational implementation of Eq. (15), in the Fourier domain because the model $T_{e}$ is uniform. The synthetic gravity and topography are then put through the spectral analysis and inversion methods which are being tested, and the recovered $T_{e}$ compared with that of the model. Since testing with just one synthetic model is statistically inadequate, Macario et al. (1995) generated 100 such models, and averaged the recovered $T_{e}$ values, a procedure which also provided a standard deviation used as a random error estimate. The results of Macario et al.’s (1995) study have been noted throughout this article, and will not be reproduced here. 
Although Macario et al. (1995) used models with uniform $T_{e}$, enabling use of the fast Fourier transform, the method was adapted by Stark et al. (2003) to synthetically model plates with spatially-variable $T_{e}$. In this case the plate flexure partial differential equation cannot be solved using the Fourier transform, and Eq. (8) must be solved in the space domain using finite differences (e.g., van Wees and Cloetingh, 1994; Stewart and Watts, 1997).

A potential problem with the Macario method was proposed by Kirby and Swain (2008), who noticed that, even though each synthetic load grid has random phase and amplitude, there can be locations in space and bands in wavenumber where the two loads are correlated. As noted in Section 6.2, the load deconvolution method makes the assumption that initial loads are statistically uncorrelated. Using the wavelet method, Kirby and Swain (2008) found that these random correlations propagate through the flexure process to the final gravity and topography, where they artificially correlate these grids. If the random correlation occurs at wavelengths close to the coherence or admittance transition, then $T_{e}$ at that spatial location is reduced. Recall (Section 6.2.6) that Macario et al. (1995) observed that increasing the degree of correlation between initial loads reduces $T_{e}$ from its true value.

Regarding the fractal dimension of the loads, Macario et al. (1995) tested values between 2.3 and 2.7, and found no influence on the final $T_{e}$ estimate. Swain and Kirby (2003a) generated loads with different fractal dimensions (the Macario study used equal fractal dimensions), and proposed that this gave rise to a load ratio that varies with wavenumber. Finally, instead of surfaces with a fractal spectrum, Simons and Olhede (2013) use those from the Matérn spectral class to generate their initial loads, whose increased number of adjustable parameters 
means that the log-log plot of their spectra is not necessarily a straight line, as it is for fractal spectra.

An issue also lies in synthetic data periodicity. The spectral synthesis method of Saupe (1988) generates surfaces that are periodic, whereas real data are non-periodic. Performing synthetic modelling on both types, Pérez-Gussinyé et al. (2004) found that use of nonperiodic data degrades $T_{e}$ recovery. Strategies to avoid data periodicity include using a spacedomain method of fractal surface generation such as the midpoint method (Saupe, 1988), or extracting sub-grids from periodic surfaces. However, as Simons and Olhede (2013) point out, the latter method effectively windows the data and so can alter the spectrum of the surface.

Instead of using synthetic modelling to check $T_{e}$ recovery, Lowry and Smith (1994) devised a method to test how well the periodogram and maximum entropy methods could recover the coherence between gravity and topography grids, when those grids had a known coherence, $\gamma_{0}^{2}(k)$. They extracted some topography from North America (a synthetic model as described above would also suffice) and Fourier transformed it to get $H$. They then formed a gravity spectrum by generating a new grid in the Fourier domain, $N$, with random phase and amplitude spectrum $|N|$, computed from the topography power spectrum via:

$$
|N(\mathbf{k})|^{2}=|H(\mathbf{k})|^{2}\left(\frac{1-\gamma_{0}^{2}(k)}{\gamma_{0}^{2}(k)}\right)
$$

The gravity grid was then formed by $G=N+H$ in the Fourier domain. They then used the two spectral analysis methods to find the coherence between $G$ and $H$, which should be $\gamma_{0}^{2}(k)$. The multitaper method was also tested in this fashion by Simons et al. $(2000,2003)$. 
Synthetic gravity and topography grids with a known admittance were generated by Crosby (2007), in order to test the admittance-recovery capabilities of the multitaper method. His scheme utilised random, fractal surfaces and the analytic equations for surface-loading and subsurface-loading admittances. In two early studies, McNutt (1983) and Diament (1985) tested the admittance-recovery capabilities of the periodogram method with different preprocessing techniques (Section 5.2). McNutt (1983) used observed topography from the US Cordillera, computing a synthetic gravity grid from this using surface-loading analytic admittance equations of known $T_{e}$. Diament (1985) used observed bathymetry profiles across a continental margin, computing the synthetic gravity using an Airy admittance model.

Finally, Braitenberg et al. (2002) and Jordan and Watts (2005) have performed synthetic testing of the space-domain convolution method (Section 6.5).

\section{Controversy: Weak Everywhere, or Sometimes Strong?}

Prior to the introduction of Forsyth’s method in 1985, nearly all $T_{e}$ studies had used (a) periodogram spectral analysis, (b) the admittance, (c) surface-loading elastic plate models, and (d) analytic inversion. The $T_{e}$ values recovered in the continents had all been low, ranging from almost Airy compensation to a couple of tens of kilometres. After the method of Forsyth (1985) and its use of (a) the Bouguer coherence, and (b) load deconvolution, continental studies began to return very high $T_{e}$ values in some places $(>100 \mathrm{~km})$ where before low values were retrieved. McKenzie and Fairhead (1997) took issue with these large $T_{e}$ values and their implication that the continental mantle lithosphere was strong. The debate that this paper initiated continues to the present day, and concerns the ultimate rheological properties of the lithosphere, sometimes known as the "jelly sandwich” (possibly strong mantle lithosphere) versus “crème brûlée” (possibly strong upper crust; weak mantle 
lithosphere) models (Burov and Watts, 2006). While the argument has reached into the realms of rheology, thermal gradient, and earthquake depth for example (e.g., Maggi et al., 2000; Jackson, 2002; Watts and Burov, 2003; Handy and Brun, 2004; McKenzie et al., 2005; Burov and Watts, 2006; Jackson et al., 2008; Burov, 2010), only the particular topic of measuring $T_{e}$ by inverse methods will be discussed here.

McKenzie and Fairhead (1997), and later McKenzie (2003), objected to blanket use of Forsyth’s method, specifically the use of the Bouguer coherence and load deconvolution. Their objection focussed on the role of unexpressed loading which Forsyth's method does not account for. Such loading, called 'model noise' here, has been detailed in Sections 3.3.9 and 6.2.5, but, briefly, its presence will cause (a) load deconvolution to model unexpressed loads as expressed loads within a high- $T_{e}$ plate, and (b) the Bouguer coherence rollover to migrate to longer wavelengths. These authors instead proposed that analytic inversion of the observed free air admittance would provide more reliable $T_{e}$ estimates in regions where unexpressed loading was present in significant amounts (Section 6.2.5). They also suggested that the free air coherence should be used as diagnostic of unexpressed loads, but as mentioned in Section 6.2.5, Kirby and Swain (2009) showed that this measure would not always be successful.

In actual fact, none of McKenzie and Fairhead's (1997) concepts was particularly new. The free air coherence was regularly used in early marine admittance studies to check for those harmonics at which the admittance could be reasonably inverted (though these studies were considering instrument noise), and Stephenson (1984) had written that the free air coherence was so low in some non-orogenic regions that the use of the spectral method could be questionable. Watts (1992) had also observed that surface loads were generally not as well 
preserved in the continents, and processes could exist that may add internal loads without topographic expression.

McKenzie and Fairhead (1997) and McKenzie (2003) estimated $T_{e}$ in several, mainly shield, regions using analytic inversion of the (multitapered) free air admittance with a combined loading model. Their results suggested that $T_{e}$ would almost never exceed $25 \mathrm{~km}$, and that expressed internal loads were very rare in most cratonic settings. They proposed that the unexpressed loading model adequately described cratonic regions, which commonly had large gravity anomalies and topography that had been subdued by erosion or sedimentation.

The community reacted strongly. Swain and Kirby (2003a) ventured that McKenzie and Fairhead's (1997) argument was not sufficient to preclude the existence of very strong plates: it could still be true that regions of large free air anomalies and subdued topography require a strong plate to prevent uplift. Many authors argued that unexpressed loads did not occur in nature. McKenzie (2003) had advanced the geological mechanism for unexpressed loading as this: correlated initial loads produce a surface topography that is subsequently eroded; the plate responds by rebounding, but the resulting topography is further eroded; the rebound/erosion process cycles until the weight of the internal loads prevents further rebound and a flat peneplain results. In contrast, Pérez-Gussinyé et al. (2004) reasoned that the rebound would instead be of sufficient magnitude to decrease the gravitational effect of the internal load, with the net result of subdued topography and gravity, both. But this state is not observed in shields, they said, where the gravity anomalies can still be large; hence large gravity anomalies indicate a plate with high $T_{e}$. 
Other authors focussed on the nature of erosion, because McKenzie and Fairhead (1997) had suggested that erosion reduces amplitude but not phase. Earlier, Forsyth (1985) had written that erosion randomises the relationship between gravity and topography, which was refined by Simons et al. (2000) to a randomisation of their phase relationship. Stephenson and Lambeck (1985b) had used models where erosion was a linear process with a rate proportional to the elevation, but Pérez-Gussinyé et al. (2004) were of the opinion that erosional power was proportional to slope rather than elevation. This meant that erosion would preferentially reduce the short wavelengths of the topography (which were due to surface initial loads) rather than the longer topographic wavelengths arising from subsurface loading; this would give the impression of subdued topography and unexpressed loads when in actuality an expressed loading model was valid. In addition, Simons et al. (2000) and Armstrong and Watts (2001) proposed that erosion occurs after the plate has cooled and $T_{e}$ has already been 'frozen in', while Watts and Burov (2003) maintained that erosion may not always reduce the landscape to a flat plain. Ultimately though, as Simons et al. (2000) wrote, there exists a considerable degree of uncertainty in the spectral characteristics of erosion, and this was probably not the topic to settle the controversy.

As detailed in Section 6.2.8, Pérez-Gussinyé et al. (2004) focussed on the spectral biases arising when analytic equations are compared against observed multitapered coherences and admittances, and found that $T_{e}$ could be underestimated. However Crosby (2007) found, from synthetic modelling, that the largest $T_{e}$ bias was only $10 \mathrm{~km}$ (for a $1000 \times 1000 \mathrm{~km}$ window), a value that could not reconcile the $>100 \mathrm{~km} T_{e}$ values from Bouguer coherence deconvolution with the <25 km values from McKenzie and Fairhead’s (1997) and McKenzie’s (2003) studies. This suggested that this particular issue, window size, was important but not the source of the discrepancy. 
Pérez-Gussinyé et al. (2004) and Pérez-Gussinyé and Watts (2005) also compared inversion of the free air admittance with the Bouguer coherence over the same study areas, with both returning $T_{e}$ values $>100 \mathrm{~km}$ in some places, and a similar spatial $T_{e}$ distribution. However they used load deconvolution with both analysis methods which meant that all loads were modelled as being expressed.

This led Kirby and Swain (2009) to perform a direct comparison of Forsyth’s and McKenzie's methods. Using the wavelet transform, they computed $T_{e}$ maps over the North American continent using by inverting the Bouguer coherence with load deconvolution, and by inverting the free air admittance using analytic equations. Their study area was large enough for analytic equations to be used, and their use of wavelets meant no windowing of the data was necessary. They found that both methods returned $T_{e}$ values $>100 \mathrm{~km}$ in the Canadian shield, with non-zero values of the (expressed) loading ratio indicating that (expressed) subsurface loads are important there. And using the normalised square of the imaginary part of the free air coherency (Section 6.2.5), Kirby and Swain (2009) found no evidence for unexpressed loading in the high- $T_{e}$ regions of the Shield, at least at wavelengths that would bias the Bouguer coherence rollover. This model-noise identification method has since been employed in several studies (Audet and Bürgmann, 2011; Mao et al., 2012; Jiménez-Díaz et al., 2014; Kirby and Swain, 2014).

The argument is still not settled, however. McKenzie (2010) recently questioned whether the high- $T_{e}$ core in the Canadian shield shown in the free air admittance $T_{e}$ map of Kirby and Swain (2009) might be an artefact due to long-wavelength contamination of the gravity and topography signals by mantle convection and glacial isostatic adjustment (GIA) effects, and 
he proposed a low $T_{e}$ value, $29 \mathrm{~km}$. Kirby and Swain (under review) acknowledged that the non-flexural phenomena are present, but called into question the particular multitaper admittance estimates of McKenzie (2010) after having conducted a detailed investigation of the spectral accuracy of both wavelets and multitapers at long wavelengths where the convective and GIA signals dominate (Kirby and Swain, 2013). Their study asserted that $T_{e}$ of the Shield is at least $80 \mathrm{~km}$.

\section{Discussion}

The techniques involved in inverse spectral methods to determine the effective elastic thickness have advanced considerably over the $40+$ years since inception, particularly in the spectral estimation process itself. From the early days of periodogram estimation, we now have at our disposal maximum entropy, multitapers and wavelets through which to calculate the admittance and coherence. Yet a very recent development calls for the abandonment of these measures altogether. In their paper, Simons and Olhede (2013) question the statistical validity of inverting ratios of products of data transforms. They ask what kind of statistical distribution do the admittance and coherence obey, are the error estimates we use appropriate, and can $T_{e}$ be realistically estimated from such ill-posed 'observables'? They suggest use of a method based on maximum-likelihood estimation theory: load deconvolution is still performed, and spectra must still be estimated, but they are not turned into a ratio such as the admittance or coherence. The resulting $T_{e}$ estimates, Simons and Olhede (2013) say, are unbiased and have minimum variance. While this is a step in the right direction, so far their method has been applied to synthetic data only, and it remains to be seen how results for real data will contribute to the current controversy on weak versus strong plates. 
Certainly the other two $T_{e}$ estimation methods (forward and rheological modelling) have not settled the argument. Elastic thickness estimates from inverse spectral methods are often at odds with those from forward modelling, and the two methods may return $T_{e}$ estimates from different eras - either the time of loading or the present-day - depending on the geological age and setting (continent or ocean) of the region (e.g., Stephenson, 1984; Zuber et al., 1989; Grotzinger and Royden, 1990; McKenzie and Fairhead, 1997; Armstrong and Watts, 2001; Watts, 2001; Billen and Gurnis, 2005; Pérez-Gussinyé and Watts, 2005; Burov and Watts, 2006; Wieczorek, 2007; McKenzie, 2010; Mouthereau et al., 2013). For example, forward models in cratons reveal the strength at the time of loading, while inverse spectral methods yield their present-day strength (e.g., Pérez-Gussinyé and Watts, 2005). So as Watts (2011) writes, it is not clear whether the two methods describe the same loading and unloading schemes.

Rheological modelling has not shed any light on the controversy either. Tesauro et al. (2012b) have compared a global continental $T_{e}$ map estimated from thermo-rheological parameters (Tesauro et al., 2012a) with one obtained from the Bouguer coherence deconvolution method using wavelets (Audet and Bürgmann, 2011). While the values in the Phanerozoic orogens are very similar, the differences in the Precambrian shields and platforms are great. Here the rheological $T_{e}$ is much less than the coherence $T_{e}$, with up to 50 km differences in some places, except for the North American craton, where the two maps have similarly large values $\left(T_{e}>100 \mathrm{~km}\right)$, and the Western Australian cratons, where the rheological $T_{e}$ is sometimes larger by a few tens of kilometres. However, Tesauro et al. (2012b) acknowledge that both methods have large uncertainties in the cratonic regions, with $T_{e}$ difficult to estimate there using both methods. 
Finally, I finish with a quote from McKenzie (2003, p4), which sums up the importance of $T_{e}$, and the importance of resolving the current controversy surrounding its values in continents. "The main consequence of this debate has been to cause most geologists and geophysicists to mistrust all estimates of $T_{e}$ from continental regions that are obtained from the spectral analysis of gravity anomalies. Such an outcome is unfortunate, since much of the tectonics of continents is likely to be controlled by the thickness of the elastic part of the lithosphere. If this argument could be decided one way or the other, much greater use could be made of measurements of gravity and topography, which are widely available.”

\section{Acknowledgements}

I thank Evgene Burov for inviting me to write this review, and the two reviewers (Tony Lowry and anonymous) for their helpful comments. The figures were plotted using GMT (Wessel and Smith, 1998). This is TIGeR publication number 513.

\section{References}

Addison, P.S., 2002. The Illustrated Wavelet Transform Handbook. Institute of Physics Publishing, Bristol, UK.

Antoine, J.-P., Murenzi, R., Vandergheynst, P., Ali, S.T., 2004. Two-dimensional Wavelets and their Relatives. Cambridge University Press, Cambridge, UK.

Armstrong, G.D., Watts, A.B., 2001. Spatial variations in $T_{e}$ in the southern Appalachians, eastern United States. Journal of Geophysical Research 106(B10), 22,009-22,026.

Audet, P., 2011. Directional wavelet analysis on the sphere: application to gravity and topography of the terrestrial planets. Journal of Geophysical Research 116, E01003, doi:10.1029/2010JE003710. 
Audet, P., 2014. Toward mapping the effective elastic thickness of planetary lithospheres from a spherical wavelet analysis of gravity and topography. Physics of the Earth and Planetary Interiors 226, 48-82.

Audet, P., Bürgmann, R., 2011. Dominant role of tectonic inheritance in supercontinent cycles. Nature Geoscience 4, 184-187.

Audet, P., Mareschal, J.-C., 2004a. Variations in elastic thickness in the Canadian Shield. Earth and Planetary Science Letters 226, 17-31.

Audet, P., Mareschal, J.-C., 2004b. Anisotropy of the flexural response of the lithosphere in the Canadian Shield. Geophysical Research Letters 31, L20601, doi:10.1029/2004GL021080.

Audet, P., Mareschal, J.-C., 2007. Wavelet analysis of the coherence between Bouguer gravity and topography: application to the elastic thickness anisotropy in the Canadian Shield. Geophysical Journal International 168, 287-298.

Audet, P., Jellinek, M., Uno, H., 2007. Mechanical controls on the deformation of continents at convergent margins. Earth and Planetary Science Letters 264, 151-166.

Banks, R.J., Swain, C.J., 1978. The isostatic compensation of East Africa. Proceedings of the Royal Society of London A 364, 331-352.

Banks, R.J., Parker, R.L., Huestis, S.P., 1977. Isostatic compensation on a continental scale: local versus regional mechanisms. Geophysical Journal of the Royal Astronomical Society $51,431-452$

Banks, R.J., Francis, S.C., Hipkin, R.G., 2001. Effects of loads in the upper crust on estimates of the elastic thickness of the lithosphere. Geophysical Journal International 145, 291-299.

Bassin, C., Laske, G., Masters, G., 2000. The current limits of resolution for surface wave tomography in North America. EOS Transactions of the American Geophysical Union 81, F897. 
Bechtel, T.D., Forsyth, D.W., Swain, C.J., 1987. Mechanisms of isostatic compensation in the vicinity of the East African Rift, Kenya. Geophysical Journal of the Royal Astronomical Society 90, 445-465.

Bechtel, T.D., Forsyth, D.W., Sharpton, V.L., Grieve, R.A.F., 1990. Variations in effective elastic thickness of the North American lithosphere. Nature 343, 636-638.

Belleguic, V., Lognonné, P., Wieczorek, M., 2005. Constraints on the Martian lithosphere from gravity and topography data. Journal of Geophysical Research 110, E11005, doi:10.1029/2005JE002437.

Bendat, J.S., Piersol, A.G., 2000. Random Data: Analysis and Measurement Procedures, third ed. Wiley and Sons, New York.

Beuthe, M., 2008. Thin elastic shells with variable thickness for lithospheric flexure of oneplate planets. Geophysical Journal International 172, 817-841.

Billen, M.I., Gurnis, M., 2005. Constraints on subducting plate strength within the Kermadec trench. Journal of Geophysical Research 110, B05407, doi:10.1029/2004JB003308.

Bracewell, R.N., 1986. The Fourier Transform and its Applications. McGraw-Hill, New York.

Braitenberg, C., Ebbing, J., Götze, H.-J., 2002. Inverse modelling of elastic thickness by convolution method - the Eastern Alps as a case example. Earth and Planetary Science Letters 202, 387-404.

Braitenberg, C., Wang, Y., Fang, J., Hsu, H.T., 2003. Spatial variations of flexure parameters over the Tibet-Quinghai plateau. Earth and Planetary Science Letters 205, 211-224.

Braitenberg, C., Wienecke, S., Wang, Y., 2006. Basement structures from satellite-derived gravity field: South China Sea ridge. Journal of Geophysical Research 111, B05407, doi:10.1029/2005JB003938. 
Burg, J.P., 1975. Maximum entropy spectral analysis. PhD thesis, Stanford University, Stanford, USA.

Burov, E.B., 2010. The equivalent elastic thickness $\left(T_{e}\right)$, seismicity and the long-term rheology of continental lithosphere: Time to burn-out “crème brûlée”? Insights from largescale geodynamic modeling. Tectonophysics 484, 4-26.

Burov, E., 2011. Lithosphere, Mechanical Properties. In: Gupta, H. (Ed), Encyclopedia of Solid Earth Geophysics. Springer, Heidelberg, pp. 693-701.

Burov, E.B., Diament, M., 1995. The effective elastic thickness $\left(T_{e}\right)$ of continental lithosphere: What does it really mean? Journal of Geophysical Research 100(B3), 39053927.

Burov, E., Diament, M., 1996. Isostasy, equivalent elastic thickness, and inelastic rheology of continents and oceans. Geology 24, 419-422.

Burov, E.B., Watts, A.B., 2006. The long-term strength of continental lithosphere: “jelly sandwich” or “crème brûlée”? GSA Today 16, 4-11.

Burov, E., Jaupart, C., Mareschal, J.C., 1998. Large-scale crustal heterogeneities and lithospheric strength in cratons. Earth and Planetary Science Letters 164, 205-219.

Canales, J.P., Dañobeitia, J.J., 1998. The Canary Islands swell: a coherence analysis of bathymetry and gravity. Geophysical Journal International 132, 479-488.

Cazenave, A., Dominh, K., 1984. Geoid heights over the Louisville Ridge (South Pacific). Journal of Geophysical Research 89(B13), 11,171-11,179.

Chapman, M.E., 1979. Techniques for interpretation of geoid anomalies. Journal of Geophysical Research 84(B8), 3793-3801.

Chen, B., Chen, C., Kaban, M.K., Du, J., Liang, Q., Thomas, M., 2013. Variations of the effective elastic thickness over China and surroundings and their relation to the lithosphere dynamics. Earth and Planetary Science Letters 363, 61-72. 
Coblentz, D., Chase, C.G., Karlstrom, K.E., van Wijk, J., 2011. Topography, the geoid, and compensation mechanisms for the southern Rocky Mountains. Geochemistry, Geophysics, Geosystems 12, Q04002, doi:10.1029/2010GC003459.

Cochran, J.R., 1979. An analysis of isostasy in the world's oceans: 2. Midocean ridge crests. Journal of Geophysical Research 84(B9), 4713-4729.

Cochran, J.R., 1980. Some remarks on isostasy and the long-term behavior of the continental lithosphere. Earth and Planetary Science Letters 46, 266-274.

Crosby, A.G., 2007. An assessment of the accuracy of admittance and coherence estimates using synthetic data. Geophysical Journal International 171, 25-54.

Dahlen, F.A., Simons, F.J., 2008. Spectral estimation on a sphere in geophysics and cosmology. Geophysical Journal International 174, 774-807.

Daly, E., Brown, C., Stark, C.P., Ebinger, C.J., 2004. Wavelet and multitaper coherence methods for assessing the elastic thickness of the Irish Atlantic margin. Geophysical Journal International 159, 445-459.

Deng, Y., Zhang, Z., Fan, W., Pérez-Gussinyé, M., 2014. Multitaper spectral method to estimate the elastic thickness of South China: implications for intracontinental deformation. Geoscience Frontiers 5, 193-203.

Detrick, R.S., Watts, A.B., 1979. An analysis of isostasy in the world's oceans: 3. Aseismic ridges. Journal of Geophysical Research 84(B7), 3637-3653.

Diament, M., 1985. Influence of method of data analysis on admittance computation. Annales de Geophysicæ 3, 785-791.

Dixon, T.H., Naraghi, M., McNutt, M.K., Smith, S.M., 1983. Bathymetric prediction from SEASAT altimeter data. Journal of Geophysical Research 88(C3), 1563-1571. 
Dorman, L.M., Lewis, B.T.R., 1970. Experimental isostasy: 1. Theory of the determination of the Earth's isostatic response to a concentrated load. Journal of Geophysical Research 75, 3357-3365.

Dorman, L.M., Lewis, B.T.R., 1972. Experimental isostasy: 3. Inversion of the isostatic Green function and lateral density changes. Journal of Geophysical Research 77, 30683077.

Ebinger, C., Hayward, N., 1996. Soft plates and hot spots: Views from Afar. Journal of Geophysical Research 101(B10), 21,859-21,876.

Ebinger, C.J., Bechtel, T.D., Forsyth, D.W., Bowin, C.O., 1989. Effective elastic plate thickness beneath the East African and Afar plateaus and dynamic compensation of the uplifts. Journal of Geophysical Research 94(B3), 2883-2901.

Farge, M., 1992. Wavelet transforms and their applications to turbulence. Annual Review of Fluid Mechanics 24, 395-457.

Flück, P., Hyndman, R.D., Lowe, C., 2003. Effective elastic thickness $T_{e}$ of the lithosphere in western Canada. Journal of Geophysical Research 108(B9), 2430, doi:10.1029/2002JB002201.

Forsyth, D.W., 1985. Subsurface loading and estimates of the flexural rigidity of continental lithosphere. Journal of Geophysical Research 90(B14), 12,623-12,632.

Fu, L.-L., and A. Cazenave, 2001. Satellite Altimetry and Earth Sciences: A Handbook of Techniques and Applications. International Geophysics Series 69, Academic Press, San Diego.

Galán, R.A., Casallas, I.F., 2010. Determination of effective elastic thickness of the Colombian Andes using satellite-derived gravity data. Earth Sciences Research Journal 14, 7-16. 
Goodwillie, A.M., Watts, A.B., 1993. An altimetric and bathymetric study of elastic thickness in the central Pacific Ocean. Earth and Planetary Science Letters 118, 311-326.

Grossman, A., Morlet, J., 1984. Decomposition of Hardy functions into square integrable wavelets of constant shape. SIAM Journal on Mathematical Analysis 15, 723-736.

Grotzinger, J., Royden, L., 1990. Elastic strength of the Slave craton at 1.9 Gyr and implications for the thermal evolution of the continents. Nature 347, 64-66.

Handy, M.R., Brun, J.-P., 2004. Seismicity, structure and strength of the continental lithosphere. Earth and Planetary Science Letters 223, 427-441.

Hanssen, A., 1997. Multidimensional multitaper spectral estimation. Signal Processing 58, 327-332.

Harry, D.L., Mickus, K.L., 1998. Gravity constraints on lithosphere flexure and the structure of the Late Paleozoic Ouachita orogen in Arkansas and Oklahoma, south central North America. Tectonics 17, 187-202.

Hartley, R., Watts, A.B., Fairhead, J.D., 1996. Isostasy of Africa. Earth and Planetary Science Letters 137, 1-18.

Heiskanen, W.A., Moritz, H., 1967. Physical Geodesy. W.H. Freeman, San Francisco. Heller, D.-A., Marquart, G., 2002. An admittance study of the Reykjanes Ridge and elevated plateaux between the Charlie-Gibbs and Senja fracture zones. Geophysical Journal International 148, 65-76.

Hyndman, R.D., Currie, C.A., Mazzotti, S., Frederiksen, A., 2009. Temperature control of continental lithosphere elastic thickness, Te vs Vs. Earth and Planetary Science Letters 277, 539-548.

Ito, G., Taira, A., 2000. Compensation of the Ontong Java Plateau by surface and subsurface loading. Journal of Geophysical Research 105(B5), 11,171-11,183. 
Jackson, J., 2002. Strength of the continental lithosphere: Time to abandon the jelly sandwich? GSA Today 12, 4-10.

Jackson, J., McKenzie, D., Priestley, K., Emmerson, B., 2008. New views on the structure and rheology of the lithosphere. Journal of the Geological Society, London 165, 453-465.

James, P.B., Zuber, M.T., Phillips, R.J., 2013. Crustal thickness and support of topography on Venus. Journal of Geophysical Research Planets 118, 859-875.

Jiménez-Díaz, A., Ruiz, J., Pérez-Gussinyé, M., Kirby, J.F., Álvarez-Gómez, J.A., Tejero, R., Capote, R., 2014. Spatial variations of effective elastic thickness of the lithosphere in Central America and surrounding regions. Earth and Planetary Science Letters 391, 55-66. Jin, Y., McNutt, M.K., Zhu, Y., 1994. Evidence from gravity and topography data for folding of Tibet. Nature 371, 669-674.

Jordan, T.A., Watts, A.B., 2005. Gravity anomalies, flexure and the elastic thickness structure of the India-Eurasia collisional system. Earth and Planetary Science Letters 236, 732-750.

Kalnins, L.M., Watts, A.B., 2009. Spatial variations in effective elastic thickness in the Western Pacific Ocean and their implications for Mesozoic volcanism. Earth and Planetary Science Letters 286, 89-100.

Karner, G.D., 1982. Spectral representation of isostatic models. BMR Journal of Australian Geology and Geophysics 7, 55-62.

Karner, G.D., Watts, A.B., 1982. On isostasy at Atlantic-type continental margins. Journal of Geophysical Research 87(B4), 2923-2948.

Karner, G.D., Watts, A.B., 1983. Gravity anomalies and flexure of the lithosphere at mountain ranges. Journal of Geophysical Research 88(B12), 10,449-10,477.

Kido, M., Yuen, D.A., Vincent, A.P., 2003. Continuous wavelet-like filter for a spherical surface and its application to localized admittance function on Mars. Physics of the Earth and Planetary Interiors 135, 1-16. 
Kirby, J.F., 2005. Which wavelet best reproduces the Fourier power spectrum? Computers and Geosciences 31, 846-864.

Kirby, J.F., Swain, C.J., 2004. Global and local isostatic coherence from the wavelet transform. Geophysical Research Letters 31, L24608, doi:10.1029/2004GL021569.

Kirby, J.F., Swain, C.J., 2006. Mapping the mechanical anisotropy of the lithosphere using a 2D wavelet coherence, and its application to Australia. Physics of the Earth and Planetary Interiors 158, 122-138.

Kirby, J.F., Swain, C.J., 2008. An accuracy assessment of the fan wavelet coherence method for elastic thickness estimation. Geochemistry, Geophysics, Geosystems 9, Q03022, doi:10.1029/2007GC001773, (Correction, Geochemistry, Geophysics, Geosystems, 9, Q05021, doi:10.1029/2008GC002071, 2008).

Kirby, J.F., Swain, C.J., 2009. A reassessment of spectral $T_{e}$ estimation in continental interiors: the case of North America. Journal of Geophysical Research 114(B8), B08401, doi:10.1029/2009JB006356.

Kirby, J.F., Swain, C.J., 2011. Improving the spatial resolution of effective elastic thickness estimation with the fan wavelet transform. Computers and Geosciences 37, 1345-1354.

Kirby, J.F., Swain, C.J., 2013. Power spectral estimates using two-dimensional Morlet-fan wavelets with emphasis on the long wavelengths: jackknife errors, bandwidth resolution and orthogonality properties. Geophysical Journal International 194, 78-99.

Kirby, J.F., Swain, C.J., 2014. The long wavelength admittance and effective elastic thickness of the Canadian shield. Journal of Geophysical Research Solid Earth 119, 51875214.

Kogan, M.G., Kostoglodov, V.V., 1981. Isostasy of fracture zones in the Atlantic Ocean. Journal of Geophysical Research 86(B10), 9248-9258. 
Kogan, M.G., McNutt, M.K., 1987. Isostasy in the USSR I: admittance data. In: Fuchs, K., Froidevaux, C. (Eds), Composition, Structure, Dynamics of the LithosphereAsthenosphere System. AGU Geodynamics Series 16, American Geophysical Union, Washington DC, pp. 301-307.

Kogan, M.G., Diament, M., Bulot, A., Balmino, G., 1985. Thermal isostasy in the South Atlantic Ocean from geoid anomalies. Earth and Planetary Science Letters 74, 280-290.

Kumar, P., Foufoula-Georgiou, E., 1994. Wavelet analysis in geophysics: an introduction. In: Kumar, P., Foufoula-Georgiou, E. (Eds), Wavelets in Geophysics. Academic Press, San Diego, pp. 1-43.

Laske, G., Masters., G., Ma, Z., Pasyanos, M., 2013. Update on CRUST1.0 - a 1-degree global model of Earth’s crust. Geophysical Research Abstracts 15, EGU2013-2658.

Lewis, B.T.R., Dorman, L.M., 1970. Experimental isostasy, 2: An isostatic model for the USA derived from gravity and topography data. Journal of Geophysical Research 75, 3367-3386.

Louden, K.E., 1981. A comparison of the isostatic response of bathymetric features in the north Pacific Ocean and Philippine Sea. Geophysical Journal of the Royal Astronomical Society 64, 393-424.

Louden, K.E., Forsyth, D.W., 1982. Crustal structure and isostatic compensation near the Kane fracture zone from topography and gravity measurements: 1 . Spectral analysis approach. Geophysical Journal of the Royal Astronomical Society 68, 725-750.

Lowry, A.R., Pérez-Gussinyé, M., 2011. The role of crustal quartz in controlling Cordilleran deformation. Nature 471, 353-357.

Lowry, A.R., Smith, R.B., 1994. Flexural rigidity of the Basin and Range-Colorado PlateauRocky Mountain transition from coherence analysis of gravity and topography. Journal of Geophysical Research 99(B10), 20,123-20,140. 
Lowry, A.R., Smith, R.B., 1995. Strength and rheology of the western U.S. Cordillera. Journal of Geophysical Research 100(B9), 17,947-17,963.

Lowry, A.R., Zhong, S., 2003. Surface versus internal loading of the Tharsis rise, Mars, Journal of Geophysical Research 108(E9), 5099, doi:10.1029/2003JE002111.

Lowry, A.R., Ribe, N.M., Smith, R.B., 2000. Dynamic elevation of the Cordillera, western United States. Journal of Geophysical Research 105(B10), 23,371-23,390.

Lyons, S.N., Sandwell, D.T., Smith, W.H.F., 2000. Three-dimensional estimation of elastic thickness under the Louisville Ridge. Journal of Geophysical Research 105(B6),13,239$13,252$.

Macario, A., Malinverno, A., Haxby, W.F., 1995. On the robustness of elastic thickness estimates obtained using the coherence method. Journal of Geophysical Research 100(B8), $15,163-15,172$.

Maggi, A., Jackson, J.A., McKenzie, D., Priestley, K., 2000. Earthquake focal depths, effective elastic thickness, and the strength of the continental lithosphere. Geology 28, 495-498.

Mao, X., Wang, Q., Liu, S., Xu, M., Wang, L., 2012. Effective elastic thickness and mechanical anisotropy of South China and surrounding regions. Tectonophysics 550-553, 47-56.

McAdoo, D.C., Sandwell, D.T., 1989. On the source of cross-grain lineations in the central Pacific gravity field. Journal of Geophysical Research 94(B7), 9341-9352.

McAdoo, D.C., Caldwell, J.G., Turcotte, D.L., 1978. On the elastic-perfectly plastic bending of the lithosphere under generalized loading with application to the Kuril Trench. Geophysical Journal of the Royal Astronomical Society 54, 11-26. 
McEwen, J.D., Hobson, M.P., Mortlock, D.J., Lasenby, A.N., 2007. Fast directional continuous spherical wavelet transform algorithms. IEEE Transactions on Signal Processing 55, 520-529.

McGovern, P.J., Solomon, S.C., Smith, D.E., Zuber, M.T., Simons, M., Wieczorek, M.A., Phillips, R.J., Neumann, G.A., Aharonson, O., Head, J.W., 2002. Localized gravity/topography admittance and correlation spectra on Mars: Implications for regional and global evolution. Journal of Geophysical Research 107(E12), 5136, doi:10.1029/2002JE001854.

McKenzie, D., 2003. Estimating $T_{e}$ in the presence of internal loads. Journal of Geophysical Research 108(B9), 2438, doi:10.1029/2002JB001766.

McKenzie, D., 2010. The influence of dynamically supported topography on estimates of $T_{e}$. Earth and Planetary Science Letters 295, 127-138.

McKenzie, D.P., Bowin, C., 1976. The relationship between bathymetry and gravity in the Atlantic Ocean. Journal of Geophysical Research 81(11), 1903-1915.

McKenzie, D., Fairhead, J.D., 1997. Estimates of the effective elastic thickness of the continental lithosphere from Bouguer and free air gravity anomalies. Journal of Geophysical Research 102(B12), 27,523-27,552.

McKenzie, D., Nimmo, F., 1997. Elastic thickness estimates for Venus from line of sight accelerations. Icarus 130, 198-216.

McKenzie, D., Jackson, J., Priestley, K., 2005. Thermal structure of oceanic and continental lithosphere. Earth and Planetary Science Letters 233, 337-349.

McNutt, M., 1979. Compensation of oceanic topography: an application of the response function technique to the Surveyor area. Journal of Geophysical Research 84(B13), 75897598. 
McNutt, M., 1980. Implications of regional gravity for state of stress in the Earth's crust and upper mantle. Journal of Geophysical Research 85(B11), 6377-6396.

McNutt, M.K., 1983. Influence of plate subduction on isostatic compensation in northern California. Tectonics 2, 399-415.

McNutt, M.K., Parker, R.L., 1978. Isostasy in Australia and the evolution of the compensating mechanism. Science 199, 773-775.

McNutt, M.K., Diament, M., Kogan, M.G., 1988. Variations of elastic plate thickness at continental thrust belts. Journal of Geophysical Research 93(B8), 8825-8838.

Mouthereau, F., Watts, A.B., Burov, E., 2013. Structure of orogenic belts controlled by lithospheric age. Nature Geoscience 6, 785-789.

Mueller, H.J., 2013. Measuring the elastic properties of natural rocks and mineral assemblages under Earth's deep crustal and mantle conditions. Journal of Geodynamics $71,25-42$.

Munk, W.H., Cartwright, D.E., 1966. Tidal spectroscopy and prediction. Philosophical Transactions of the Royal Society of London A 259, 533-581.

Nair, R.R., Singh, Y., Trivedi, D., Kandpal, S.C., 2012. Anisotropy in the flexural response of the Indian Shield. Tectonophysics 532-535, 193-204.

Ojeda, G.Y., Whitman, D., 2002. Effect of windowing on lithosphere elastic thickness estimates obtained via the coherence method: results from northern South America. Journal of Geophysical Research 107(B11), 2275, doi:10.1029/2000JB000114.

Parker, R.L., 1972. The rapid calculation of potential anomalies. Geophysical Journal of the Royal Astronomical Society 31, 447-455.

Parker, R.L., McNutt, M.K., 1980. Statistics for the one-norm misfit measure. Journal of Geophysical Research 85(B8), 4429-4430. 
Percival, D.P., Walden, A.T., 1993. Spectral Analysis for Physical Applications. Cambridge University Press, Cambridge.

Pérez-Gussinyé, M., Watts, A.B., 2005. The long-term strength of Europe and its implications for plate-forming processes. Nature 436, 381-384.

Pérez-Gussinyé, M., Lowry, A.R., Watts, A.B., Velicogna, I., 2004. On the recovery of effective elastic thickness using spectral methods: Examples from synthetic data and from the Fennoscandian Shield. Journal of Geophysical Research 109(B10), B10409, doi:10.1029/2003JB002788.

Pérez-Gussinyé, M., Lowry, A.R., Watts, A.B., 2007. Effective elastic thickness of South America and its implications for intracontinental deformation. Geochemistry, Geophysics, Geosystems 8, Q05009, doi:10.1029/2006GC001511.

Pérez-Gussinyé, M., Lowry, A.R., Phipps-Morgan, J., Tassara, A., 2008. Effective elastic thickness variations along the Andean margin and their relationship to subduction geometry. Geochemistry, Geophysics, Geosystems 9, Q02003, doi:10.1029/2007GC001786.

Pérez-Gussinyé, M., Swain, C.J., Kirby, J.F., Lowry, A.R., 2009a. Spatial variations of the effective elastic thickness, $T_{e}$, using multitaper spectral estimation and wavelet methods: examples from synthetic data and application to South America. Geochemistry, Geophysics, Geosystems 10, Q04005, doi:10.1029/2008GC002229.

Pérez-Gussinyé, M., Metois, M., Fernández, M., Vergés, J., Fullea, J., Lowry, A.R., 2009b. Effective elastic thickness of Africa and its relationship to other proxies for lithospheric structure and surface tectonics. Earth and Planetary Science Letters 287, 152-167.

Pilkington, M., 1991. Mapping elastic lithospheric thickness variations in Canada. Tectonophysics 190, 283-297. 
Poudjom Djomani, Y.H., Nnange, J.M., Diament, M., Ebinger, C.J., Fairhead, J.D., 1995. Effective elastic thickness and crustal thickness variations in west central Africa inferred from gravity data. Journal of Geophysical Research 100(B11), 22,047-22,070.

Poudjom Djomani, Y.H., Fairhead, J.D., Griffin, W.L., 1999. The flexural rigidity of Fennoscandia: reflection of the tectonothermal age of the lithospheric mantle. Earth and Planetary Science Letters 174, 139-154.

Poudjom Djomani Y.H., Griffin, W.L., O’Reilly, S.Y., Doyle, B.J., 2005. Lithospheric domains and controls on kimberlite emplacement, Slave province, Canada: Evidence from elastic thickness and upper mantle composition. Geochemistry, Geophysics, Geosystems 6, Q10006, doi:10.1029/2005GC000978.

Press, W.H., Teukolsky, S.A., Vetterling, W.T., Flannery, B.P., 1992. Numerical Recipes in Fortran 77, second ed. Cambridge University Press, Cambridge.

Rajesh, R.S., Mishra, D.C., 2004. Lithospheric thickness and mechanical strength of the Indian shield. Earth and Planetary Science Letters 225, 319-328.

Ratheesh-Kumar, R.T., Windley, B.F., Sajeev, K., 2014. Tectonic inheritance of the Indian Shield: new insights from its elastic thickness structure. Tectonophysics 615-616, 40-52. Ribe, N.M., 1982. On the interpretation of frequency response functions for oceanic gravity and bathymetry. Geophysical Journal of the Royal Astronomical Society 70, 273-294.

Ribe, N.M., Watts, A.B., 1982. The distribution of intraplate volcanism in the Pacific Ocean basin: a spectral approach. Geophysical Journal of the Royal Astronomical Society 71, 333-362.

Sambridge, M., 1999a. Geophysical inversion with a Neighbourhood Algorithm - I. Searching a parameter space. Geophysical Journal International 138, 479-494. Sambridge, M., 1999b. Geophysical inversion with a Neighbourhood Algorithm - II. Appraising the ensemble. Geophysical Journal International 138, 727-746. 
Sandwell, D.T., 1982. Thermal isostasy: response of a moving lithosphere to a distributed heat source. Journal of Geophysical Research 87(B2), 1001-1014.

Sandwell, D.T., Poehls, K.A., 1980. A compensation mechanism for the central Pacific. Journal of Geophysical Research 85(B7), 3751-3758.

Saupe, D., 1988. Algorithms for random fractals. In: Peitgen, H.-O., Saupe, D. (Eds.), The Science of Fractal Images. Springer, New York, pp. 71-136.

Shin, Y.H., Xu, H., Braitenberg, C., Fang, J., Wang, Y., 2007. Moho undulations beneath Tibet from GRACE-integrated gravity data. Geophysical Journal International 170, 971985.

Simons, F.J., Olhede, S.C., 2013. Maximum-likelihood estimation of lithospheric flexural rigidity, initial-loading fraction and load correlation, under isotropy. Geophysical Journal International 193, 1300-1342.

Simons, F.J., van der Hilst, R.D., 2002. Age-dependent seismic thickness and mechanical strength of the Australian lithosphere. Geophysical Research Letters 29, doi:10.1029/2002GL014962.

Simons, F.J., Wang, D.V., 2011. Spatiospectral concentration in the Cartesian plane. International Journal on Geomathematics 2, 1-36.

Simons, F.J., Zuber, M.T., Korenaga, J., 2000. Isostatic response of the Australian lithosphere: Estimation of effective elastic thickness and anisotropy using multitaper spectral analysis. Journal of Geophysical Research 105(B8), 19,163-19,184.

Simons, F.J., van der Hilst, R.D., Zuber, M.T., 2003. Spatiospectral localization of isostatic coherence anisotropy in Australia and its relation to seismic anisotropy: Implications for lithospheric deformation. Journal of Geophysical Research 108(B5), 2250, doi:10.1029/2001JB000704. 
Simons, F.J., Dahlen, F.A., Wieczorek, M.A., 2006. Spatiospectral concentration on a sphere. SIAM Review 48, 504-536.

Simons, M., Hager, B.H., Solomon, S.C., 1994. Global variations in the geoid/topography admittance of Venus. Science 264, 798-803

Simons, M., Solomon, S.C., Hager, B.H., 1997. Localization of gravity and topography: constraints on the tectonics and mantle dynamics of Venus. Geophysical Journal International 131, 24-44.

Slepian, D., 1978. Prolate spheroidal wave functions, Fourier analysis, and uncertainty - V: the discrete case. Bell System Technical Journal 57, 1371-1430.

Stark, C.P., Stewart, J., Ebinger, C.J., 2003. Wavelet transform mapping of effective elastic thickness and plate loading: Validation using synthetic data and application to the study of southern African tectonics. Journal of Geophysical Research 108(B12), 2558, doi:10.1029/2001JB000609.

Stephenson, R., 1984. Flexural models of continental lithosphere based on the long-term erosional decay of topography. Geophysical Journal of the Royal Astronomical Society $77,385-413$

Stephenson, R., Beaumont, C., 1980. Small-scale convection in the upper mantle and the isostatic response of the Canadian shield. In: Davies, P.A., Runcorn, S.K. (Eds.), Mechanisms of Continental Drift and Plate Tectonics. Academic Press, San Diego, pp. 111-122.

Stephenson, R., Lambeck, K., 1985a. Erosion-isostatic rebound models for uplift: an application to south-eastern Australia. Geophysical Journal of the Royal Astronomical Society 82, 31-55. 
Stephenson, R., Lambeck, K., 1985b. Isostatic response of the lithosphere with in-plate stress: application to central Australia. Journal of Geophysical Research 90(B10), 85818588.

Stewart, J., Watts, A.B., 1997. Gravity anomalies and spatial variations of flexural rigidity at mountain ranges. Journal of Geophysical Research 102(B3), 5327-5352.

Swain, C.J., Kirby, J.F., 2003a. The effect of 'noise' on estimates of the elastic thickness of the continental lithosphere by the coherence method. Geophysical Research Letters 30, 1574, doi:10.1029/2003GL017070.

Swain, C.J., Kirby, J.F., 2003b. The coherence method using a thin anisotropic elastic plate model. Geophysical Research Letters 30, 2014, doi:10.1029/2003GL018350.

Swain, C.J., Kirby, J.F., 2006. An effective elastic thickness map of Australia from wavelet transforms of gravity and topography using Forsyth’s method. Geophysical Research Letters 33, L02314, doi:10.1029/2005GL025090.

Szilard, R., 1974. Theory and Analysis of Plates. Prentice-Hall, Englewood Cliffs, New Jersey.

Tarantola, A., 1987. Inverse Problem Theory. Elsevier, Amsterdam.

Tassara, A., Swain, C., Hackney, R., Kirby, J., 2007. Elastic thickness structure of South America estimated using wavelets and satellite-derived gravity data. Earth and Planetary Science Letters 253, 17-36.

Tesauro, M., Kaban, M.K., Cloetingh, S.A.P.L., 2012a. Global strength and elastic thickness of the lithosphere. Global and Planetary Change 90-91, 51-57.

Tesauro, M., Audet, P., Kaban, M.K., Bürgmann, R., Cloetingh, S., 2012b. The effective elastic thickness of the continental lithosphere: Comparison between rheological and inverse approaches. Geochemistry, Geophysics, Geosystems 13, Q09001, doi:10.1029/2012GC004162. 
Thomson, D.J., 1982. Spectrum estimation and harmonic-analysis. Proceedings of the IEEE 70, 1055-1096.

Thomson, D.J., Chave, A.D., 1991. Jackknifed error estimates for spectra, coherences, and transfer functions. In: Haykin, S. (Ed.), Advances in Spectrum Analysis and Array Processing 1. Prentice Hall, Englewood Cliffs, N.J., pp. 58-113.

Timoshenko, S.P., Woinowsky-Krieger, S., 1959. Theory of Plates and Shells, third ed. McGraw-Hill, New York.

Tiwari, V.M., Grevemeyer, I., Singh, B., Phipps Morgan, J., 2007. Variation of effective elastic thickness and melt production along the Deccan-Reunion hotspot track. Earth and Planetary Science Letters 264, 9-21.

Turcotte, D.L., Schubert, G., 2002. Geodynamics, second ed. Cambridge University Press, Cambridge.

Turcotte, D.L., McAdoo, D.C., Caldwell, J.G., 1978. An elastic-perfectly plastic analysis of the bending of the lithosphere at a trench. Tectonophysics 47, 193-205.

Turcotte, D.L., Willemann, R.J., Haxby, W.F., Norberry, J., 1981. Role of membrane stresses in support of planetary topography. Journal of Geophysical Research 86, 3951-3959.

van Wees, J.D., Cloetingh, S., 1994. A finite-difference technique to incorporate spatial variations in rigidity and planar faults into 3-D models for lithospheric flexure. Geophysical Journal International 117, 179-195.

Walcott, R.I., 1976. Lithospheric flexure, analysis of gravity anomalies, and the propagation of seamount chains. In: Sutton, G.H., Manghnani, M.H., Moberly, R. (Eds), The Geophysics of the Pacific Ocean Basin and its Margin, AGU Geophysical Monograph Series 19, 431-438, American Geophysical Union, Washington DC.

Wang, Y., Mareschal, J.-C., 1999. Elastic thickness of the lithosphere in the Central Canadian Shield. Geophysical Research Letters 26, 3033-3035. 
Watts, A.B., 1978. An analysis of isostasy in the world's oceans: 1. Hawaiian-Emperor seamount chain. Journal of Geophysical Research 83(B12), 5989-6004.

Watts, A.B., 1979. On geoid heights derived from Geos 3 altimeter data along the HawaiianEmperor seamount chain. Journal of Geophysical Research 84(B8), 3817-3826.

Watts, A.B., 1992. The effective elastic thickness of the lithosphere and the evolution of foreland basins. Basin Research 4, 169-178.

Watts, A.B., 2001. Isostasy and Flexure of the Lithosphere. Cambridge University Press, Cambridge.

Watts, A.B., 2011. Isostasy. In: Gupta, H. (Ed), Encyclopedia of Solid Earth Geophysics. Springer, Heidelberg, pp. 647-661.

Watts, A.B., Burov, E.B., 2003. Lithospheric strength and its relationship to the elastic and seismogenic layer thickness. Earth and Planetary Science Letters 213, 113-131.

Watts, A.B., Ribe, N.M., 1984. On geoid heights and flexure of the lithosphere at seamounts. Journal of Geophysical Research 89(B13), 11,152-11,170.

Watts, A.B., Bodine, J.H., Ribe, N.M., 1980. Observations of flexure and the geological evolution of the Pacific Ocean basin. Nature 283, 532-537.

Watts, A.B., Cochran, J.R., Patriat, P., Doucouré, M., 1985. A bathymetry and altimetry profile across the Southwest Indian Ridge crest at $31^{\circ} \mathrm{S}$ latitude. Earth and Planetary Science Letters 73, 129-139.

Watts, A.B., Weissel, J.K., Duncan, R.A., Larson, R.L., 1988. Origin of the Louisville Ridge and its relationship to the Eltanin Fracture Zone system. Journal of Geophysical Research 93(B4), 3051-3077.

Watts, A.B., Sandwell, D.T., Smith, W.H.F., Wessel, P., 2006. Global gravity, bathymetry, and the distribution of submarine volcanism through space and time. Journal of Geophysical Research 111, B08408, doi:10.1029/2005JB004083. 
Wessel, P., Smith, W.H.F., 1998. New, improved version of Generic Mapping Tools released, EOS: Transactions of the American Geophysical Union 79, 579.

Wieczorek, M.A., 2007. Gravity and topography of the terrestrial planets. In: Schubert, G. (Ed.), Treatise on Geophysics 10. Elsevier, Amsterdam, pp. 165-206.

Wieczorek, M.A., Simons, F.J., 2005. Localized spectral analysis on the sphere. Geophysical Journal International 162, 655-675.

Wienecke, S., Braitenberg, C., Götze, H.-J., 2007. A new analytical solution estimating the flexural rigidity in the Central Andes. Geophysical Journal International 169, 789-794.

Zuber, M.T., Bechtel, T.D., Forsyth, D.W., 1989. Effective elastic thickness of the lithosphere and the mechanisms of isostatic compensation in Australia. Journal of Geophysical Research 94(B7), 9353-9367. 


\begin{tabular}{|c|c|c|}
\hline Continent & Spectra & Study \\
\hline \multirow{4}{*}{ North America } & PG & $\begin{array}{l}\text { Bechtel et al. (1990); Pilkington (1991); Poudjom Djomani } \\
\text { et al. (2005) }\end{array}$ \\
\hline & ME & $\begin{array}{l}\text { Lowry and Smith (1994, 1995); Wang and Mareschal } \\
\text { (1999); Lowry et al. (2000); Armstrong and Watts (2001); } \\
\text { Flück et al. (2003); Audet and Mareschal (2004a); } \\
\text { Hyndman et al. (2009) }\end{array}$ \\
\hline & MT & $\begin{array}{l}\text { Audet and Mareschal (2004b); Audet et al. (2007); Lowry } \\
\text { and Pérez-Gussinyé (2011) }\end{array}$ \\
\hline & WT & $\begin{array}{l}\text { Audet and Mareschal (2007); Audet et al. (2007); Kirby } \\
\text { and Swain (2009) }{ }^{1} \text {; Kirby and Swain (2014) }\end{array}$ \\
\hline $\begin{array}{l}\text { Central } \\
\text { America }\end{array}$ & MT, WT & Jiménez-Díaz et al. (2014) \\
\hline \multirow{3}{*}{ South America } & MT & Pérez-Gussinyé et al. (2007, 2008, 2009a) \\
\hline & WT & Tassara et al. (2007); Kirby and Swain (2011) \\
\hline & SC & Wienecke et al. (2007) \\
\hline \multirow{3}{*}{ Europe } & PG & Poudjom Djomani et al. (1999) \\
\hline & MT & $\begin{array}{l}\text { Pérez-Gussinyé et al. (2004)²; Pérez-Gussinyé and Watts } \\
(2005)^{2}\end{array}$ \\
\hline & SC & Braitenberg et al. (2002) \\
\hline \multirow{3}{*}{ Africa } & PG & $\begin{array}{l}\text { Ebinger et al. (1989); Poudjom Djomani et al. (1995); } \\
\text { Ebinger and Hayward (1996); Hartley et al. (1996) }\end{array}$ \\
\hline & MT & Pérez-Gussinyé et al. (2009b) \\
\hline & WT & Stark et al. (2003) \\
\hline
\end{tabular}




\begin{tabular}{|c|c|c|}
\hline \multirow{4}{*}{ Asia } & PG & Kogan and McNutt $(1987)^{3}$ \\
\hline & MT & $\begin{array}{l}\text { Rajesh and Mishra (2004) }{ }^{4} \text {; Nair et al. (2012); Chen et al. } \\
\text { (2013); Deng et al. (2014) }\end{array}$ \\
\hline & WT & Mao et al. (2012); Ratheesh-Kumar et al. (2014) \\
\hline & SC & $\begin{array}{l}\text { Braitenberg et al. (2003); Jordan and Watts (2005); } \\
\text { Braitenberg et al. (2006) }\end{array}$ \\
\hline \multirow{3}{*}{ Australia } & PG & Zuber et al. (1989) \\
\hline & MT & Simons and van der Hilst (2002) ${ }^{4}$ \\
\hline & WT & Swain and Kirby (2006) \\
\hline \multirow{2}{*}{ Global } & Compilation & Watts (2001); Mouthereau et al. (2013) \\
\hline & WT & Audet and Bürgmann (2011) ${ }^{6}$ \\
\hline Atlantic Ocean & MT, WT & Daly et al. (2004) \\
\hline Pacific Ocean & PG & Kalnins and Watts (2009) \\
\hline
\end{tabular}

Table 1. Studies that have created maps of the spatial variation of $T_{e}$, grouped according to continent and the spectral estimation methods: PG, periodogram, usually mirrored; ME, maximum entropy; MT, multitapers; WT, wavelet transform; SC, space-domain convolution. All studies used the Bouguer coherence deconvolution method (BCD), except the following: ${ }^{1} \mathrm{BCD}$ and FQU; ${ }^{2} \mathrm{BCD}$ and FQD; ${ }^{3} \mathrm{BQU} ;{ }^{4} \mathrm{CTW} ;{ }^{5} \mathrm{FQU}$; where F represents free air anomalies, B Bouguer anomalies; C coherence, Q admittance; D load deconvolution, U uniform- $f$ inversion; CTW represents mapping of the coherence transition wavelength, with no inversion for $T_{e}{ }^{6}$ continental $T_{e}$ only. 


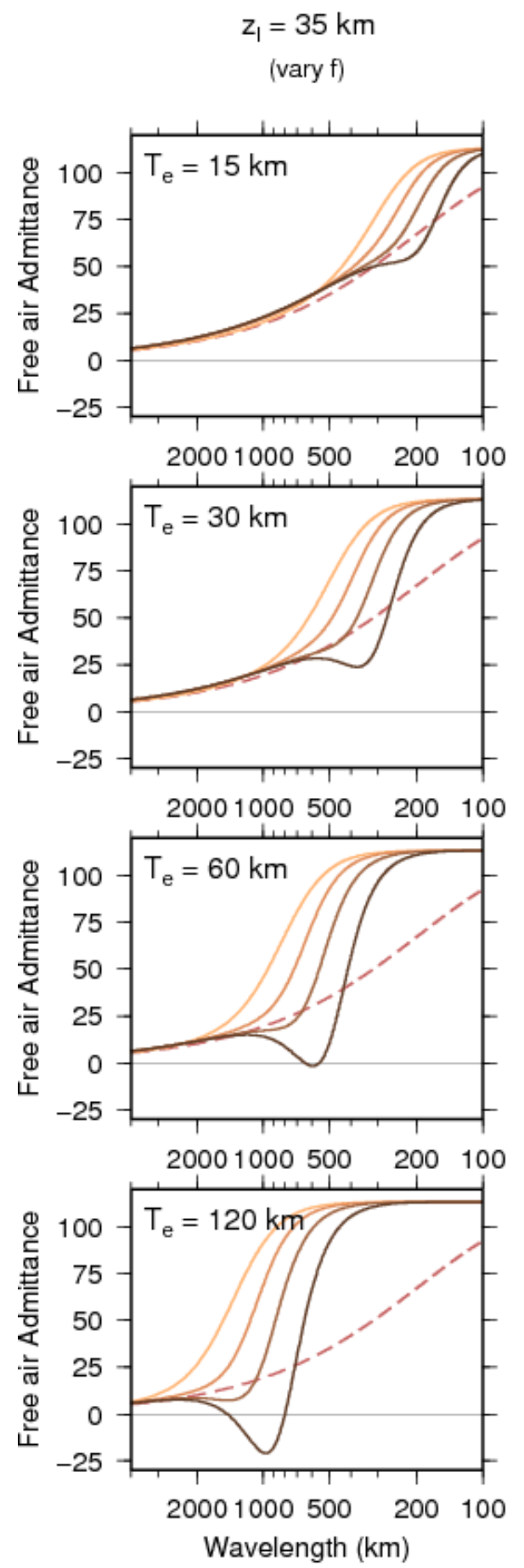

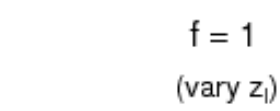
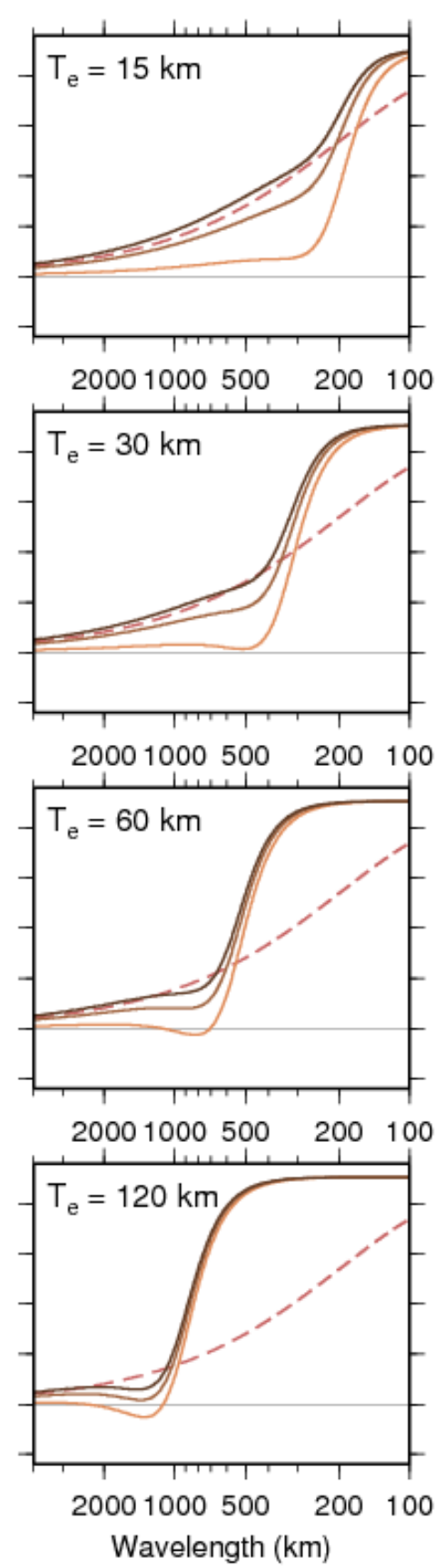

$\mathrm{T}_{\mathrm{e}}=30 \mathrm{~km}$

(vary $z_{1}$ )
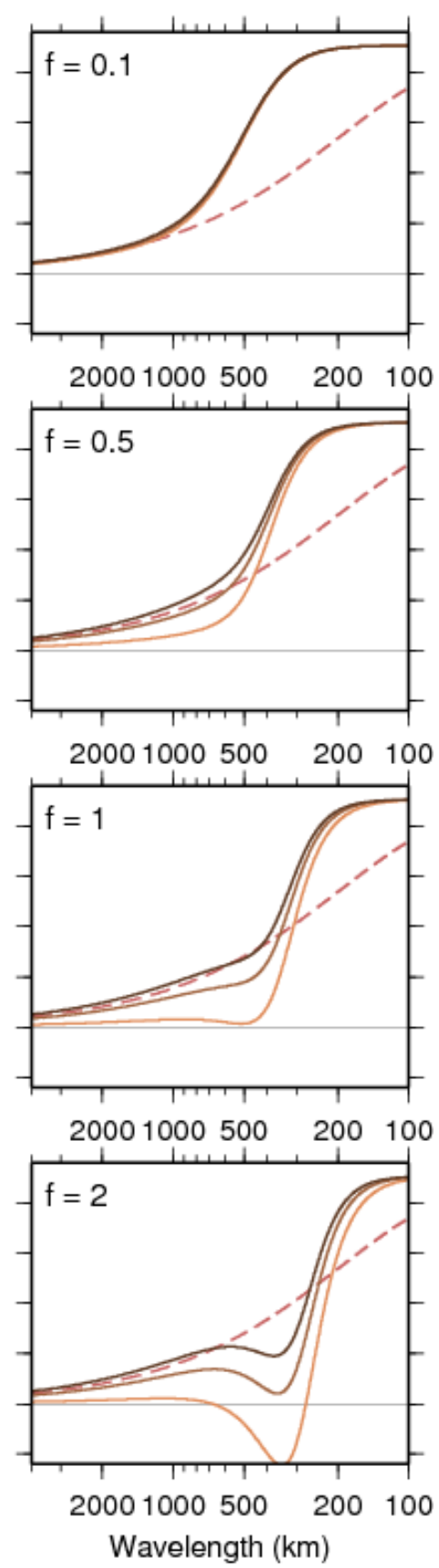

Fig. 1. Theoretical free air admittance curves for various indicated values of $T_{e}, f$ and depth to internal load $\left(z_{l}\right)$, where $f$ is uniform, i.e., has the quoted value at all wavenumbers. The shading of the curves gets darker as the quantity increases. In the left-hand column (constant $z_{l}$ ) the curves show the variation with $f=0.1,0.5,1$ and 2 . In the middle column (constant $f$ ) the curves show the variation with $z_{l}=10,25$ and $35 \mathrm{~km}$. In the right-hand column (constant 
$T_{e}$ ) the curves show the variation with $z_{l}=10,25$ and $35 \mathrm{~km}$. In all plots the dashed red curve shows the Airy isostasy signal $\left(T_{e}=0, f=0\right)$ for $z_{l}=35 \mathrm{~km}$. Units of the admittance are $\mathrm{mGal} / \mathrm{km}$. To obtain the Bouguer admittance, subtract $2 \pi \mathcal{G} \rho_{1}$. The model uses a three-layer crust of densities (top to bottom) 2700, 2800 and $2900 \mathrm{~kg} / \mathrm{m}^{3}$, and depths to base of 10, 25 and $35 \mathrm{~km}$; the mantle has density $3400 \mathrm{~kg} / \mathrm{m}^{3}$. These plots are for continental loading. In the oceans the $\mathrm{e}^{-k d}$ term, for ocean depth $d$, dominates at short wavelengths, and the admittance decreases to zero at very short wavelengths. 


$$
\begin{gathered}
z_{1}=35 \mathrm{~km} \\
(\text { vary } f)
\end{gathered}
$$
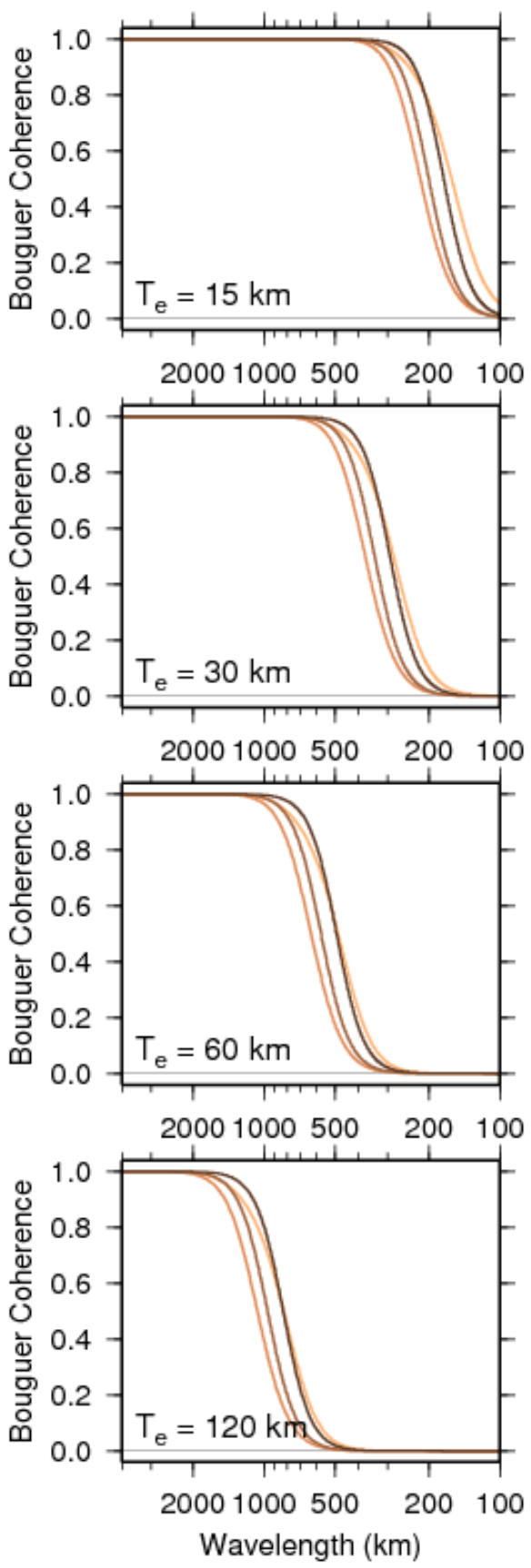

$f=1$

(vary $\left.z_{1}\right)$
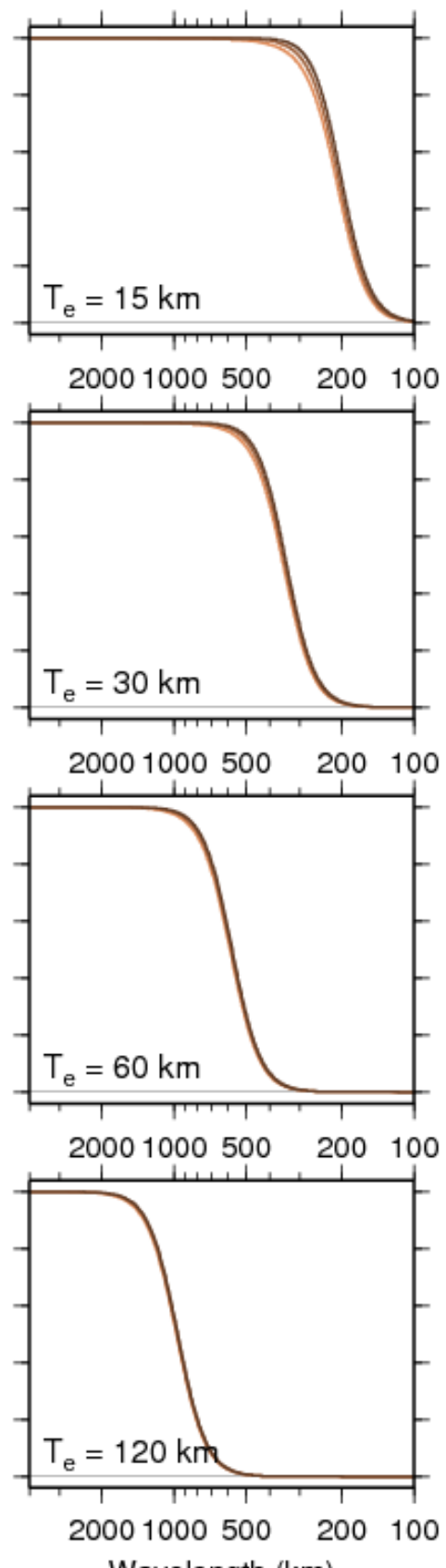

Wavelength $(\mathrm{km})$
$\mathrm{T}_{\mathrm{e}}=30 \mathrm{~km}$

(vary $\left.z_{1}\right)$
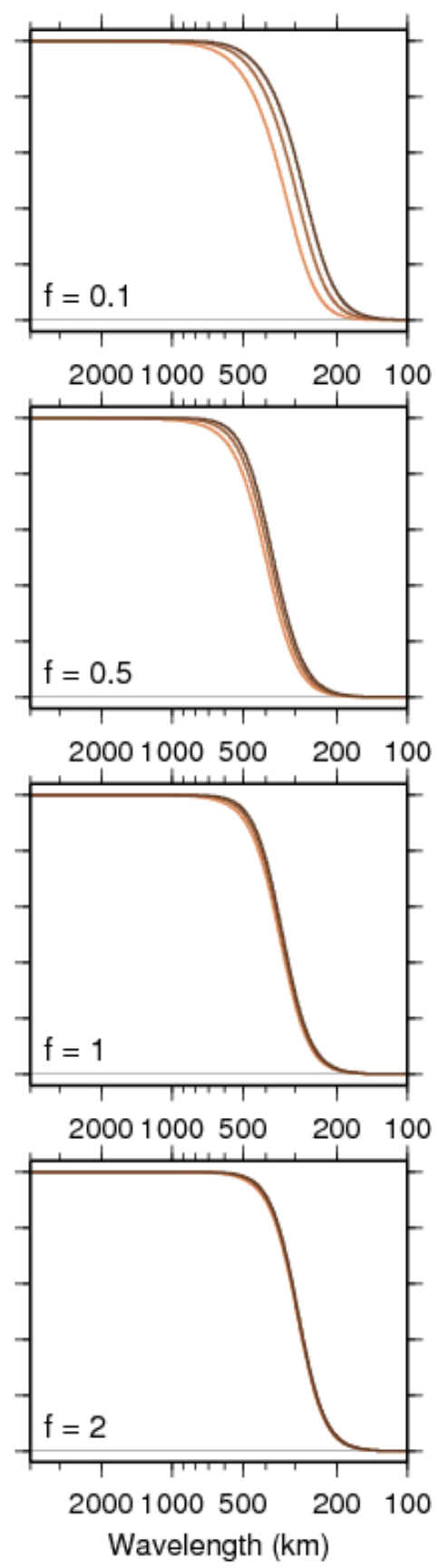

Fig. 2. Theoretical Bouguer coherence curves for various indicated values of $T_{e}, f$ and depth to internal load $\left(z_{l}\right)$. The shading of the curves gets darker as the quantity increases. In the left-hand column (constant $z_{l}$ ) the curves show the variation with $f=0.1,0.5,1$ and 2 . In the middle column (constant $f$ ) the curves show the variation with $z_{l}=10,25$ and $35 \mathrm{~km}$. In the 
right-hand column (constant $T_{e}$ ) the curves show the variation with $z_{l}=10,25$ and $35 \mathrm{~km}$. Model parameters as for Fig. 1. 


$$
\begin{aligned}
& \mathrm{z}_{1}=35 \mathrm{~km} \\
& (\operatorname{vary} \mathrm{f})
\end{aligned}
$$
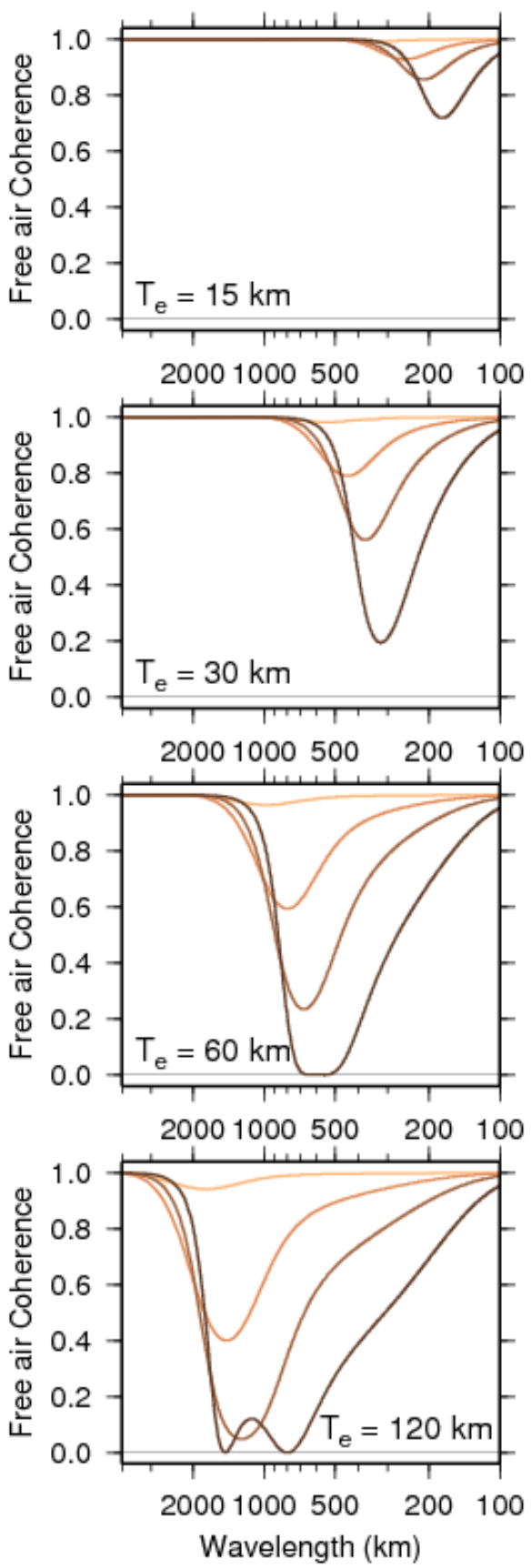

$f=1$

(vary $\left.z_{1}\right)$
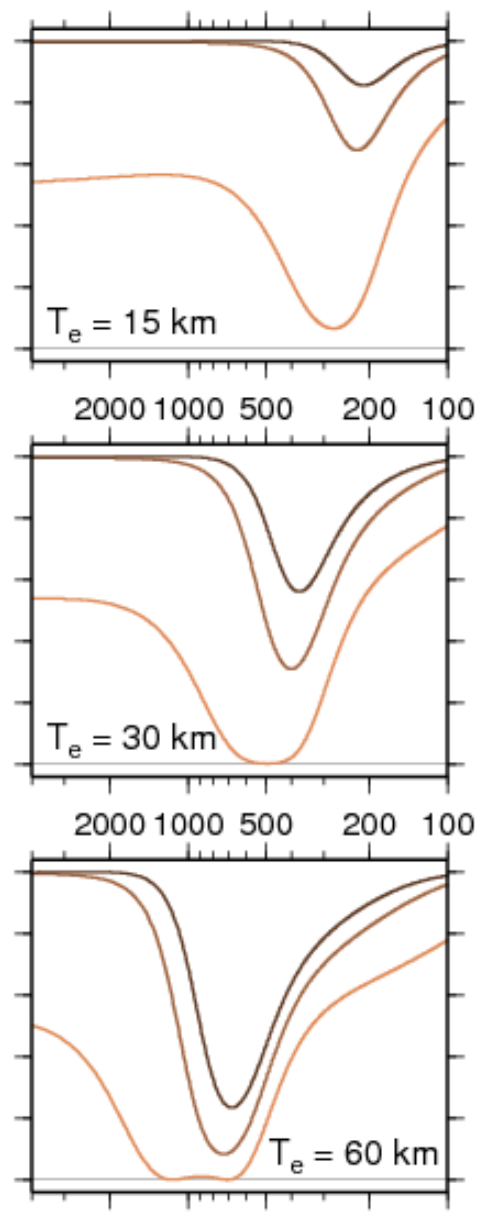

20001000500200100

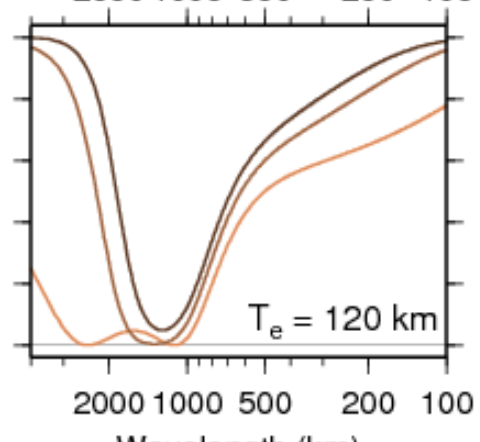

Wavelength $(\mathrm{km})$
$\mathrm{T}_{\mathrm{e}}=30 \mathrm{~km}$

(vary $\left.z_{1}\right)$
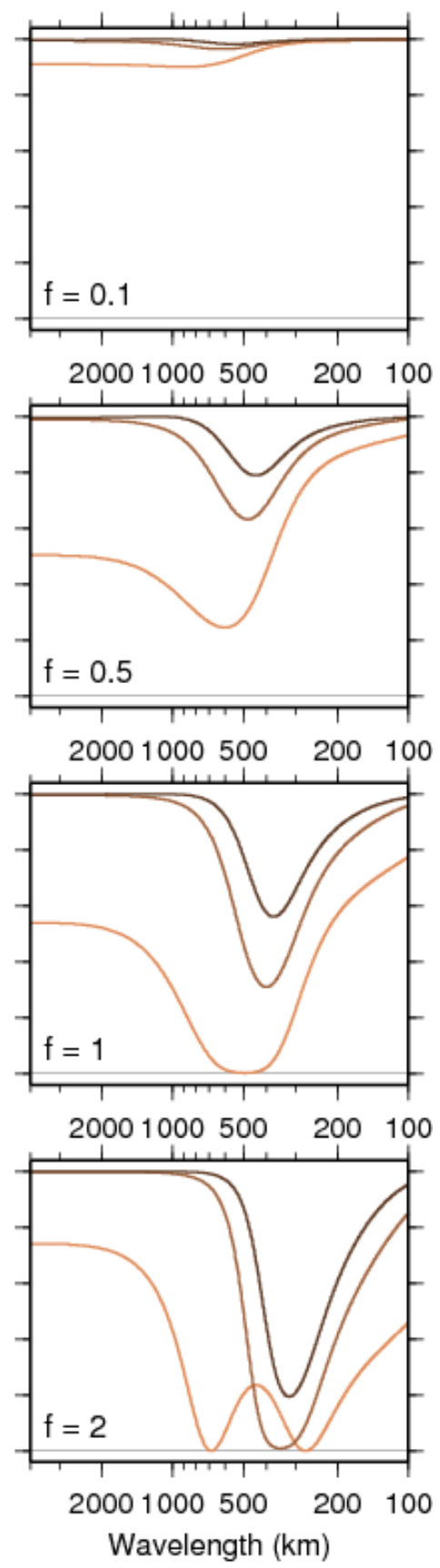

Fig. 3. Theoretical free air coherence curves for various indicated values of $T_{e}, f$ and depth to internal load $\left(\mathrm{z}_{l}\right)$. The shading of the curves gets darker as the quantity increases. In the lefthand column (constant $z_{l}$ ) the curves show the variation with $f=0.1,0.5,1$ and 2 . In the middle column (constant $f$ ) the curves show the variation with $z_{l}=10,25$ and $35 \mathrm{~km}$. In the 
right-hand column (constant $T_{e}$ ) the curves show the variation with $z_{l}=10,25$ and $35 \mathrm{~km}$. Model parameters as for Fig. 1. 
$z_{1}=35 \mathrm{~km}$

(vary $f$ )
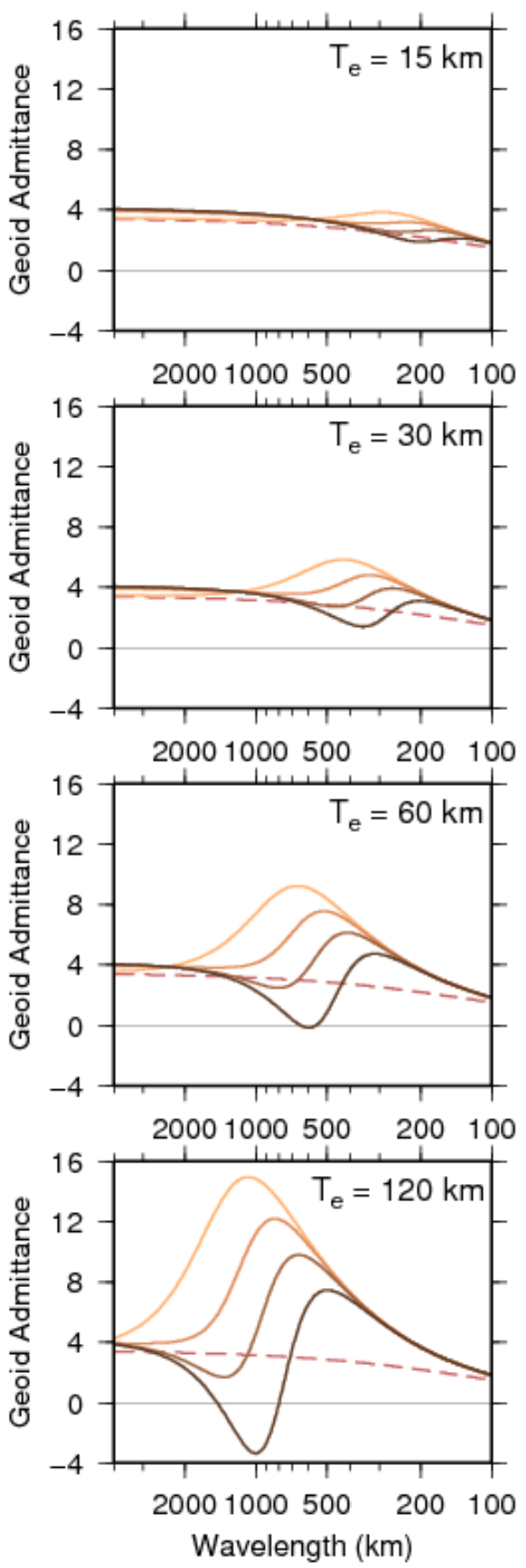

$f=1$

(vary $\left.z_{1}\right)$
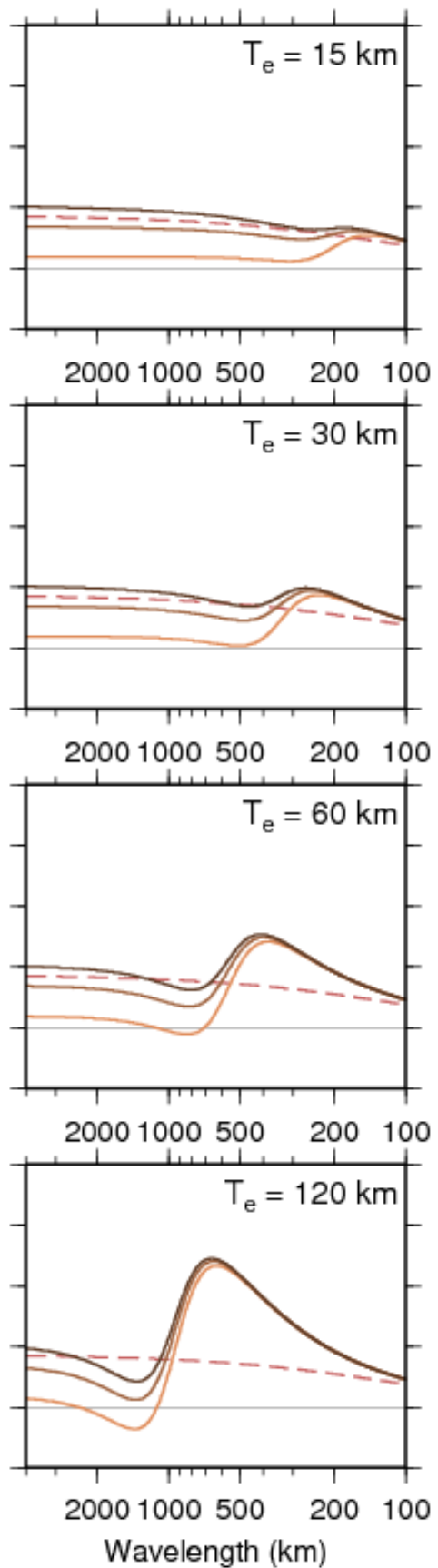

$\mathrm{T}_{\mathrm{e}}=30 \mathrm{~km}$

(vary $z_{1}$ )
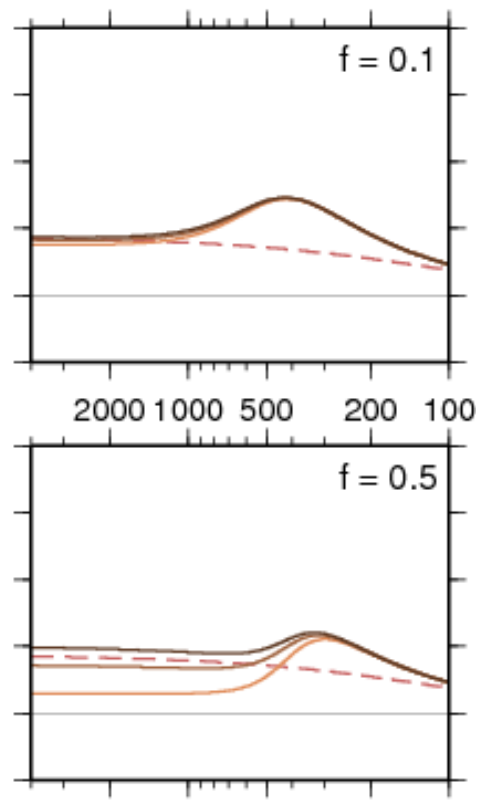

20001000500200100
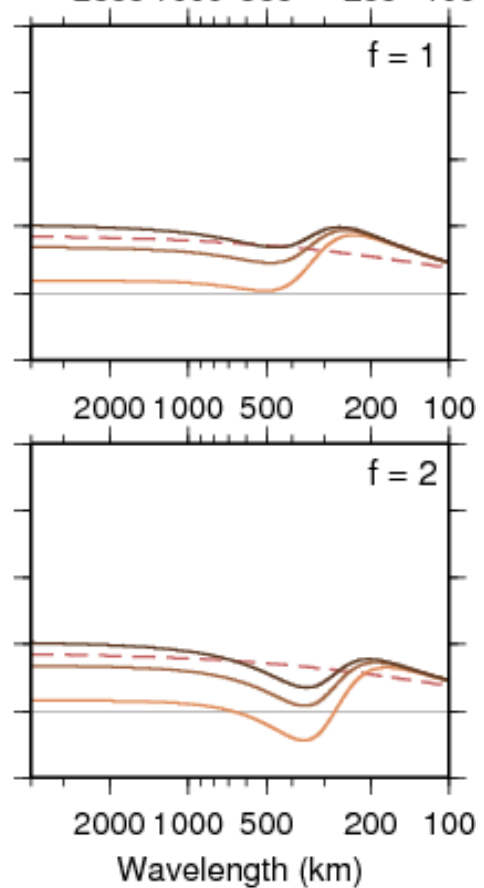

Fig. 4. Theoretical geoid admittance curves for various indicated values of $T_{e}, f$ and depth to internal load $\left(z_{l}\right)$. The shading of the curves gets darker as the quantity increases. In the lefthand column (constant $z_{l}$ ) the curves show the variation with $f=0.1,0.5,1$ and 2 . In the middle column (constant $f$ ) the curves show the variation with $z_{l}=10,25$ and $35 \mathrm{~km}$. In the 
right-hand column (constant $T_{e}$ ) the curves show the variation with $z_{l}=10,25$ and $35 \mathrm{~km}$. In all plots the dashed red curve shows the Airy isostasy signal $\left(T_{e}=0, f=0\right)$ for $\mathrm{z}_{l}=35 \mathrm{~km}$. Units of the admittance are $\mathrm{m} / \mathrm{km}$. Model parameters as for Fig. 1. 

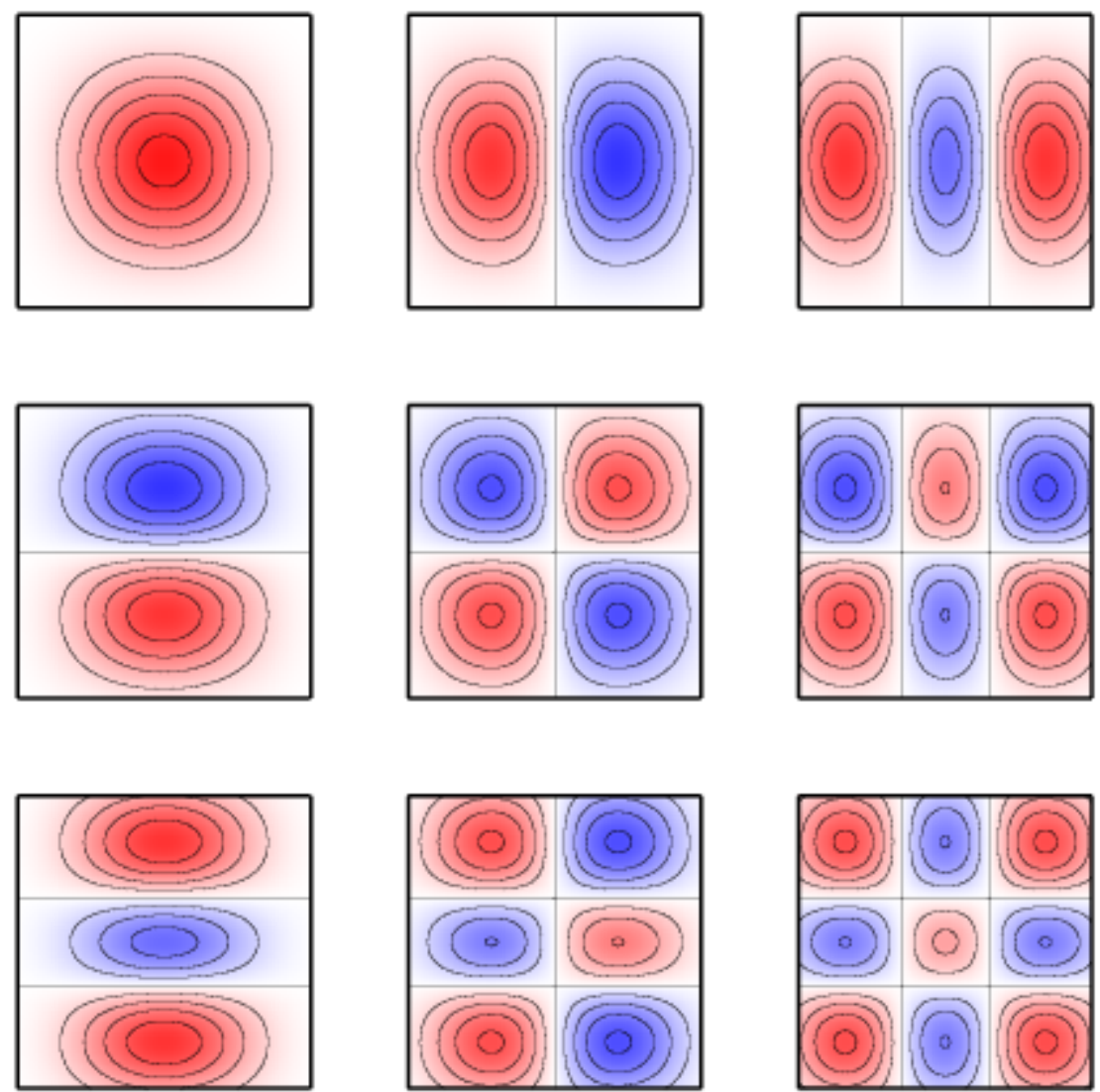

Fig. 5. The 9 2D Slepian tapers in the space domain corresponding to a bandwidth parameter of NW $=2$. The data are multiplied by each taper and then Fourier transformed, giving 9 estimates of the data's power spectrum; these are then averaged to give the power spectrum of the data. Red colours are positive, blue negative. 

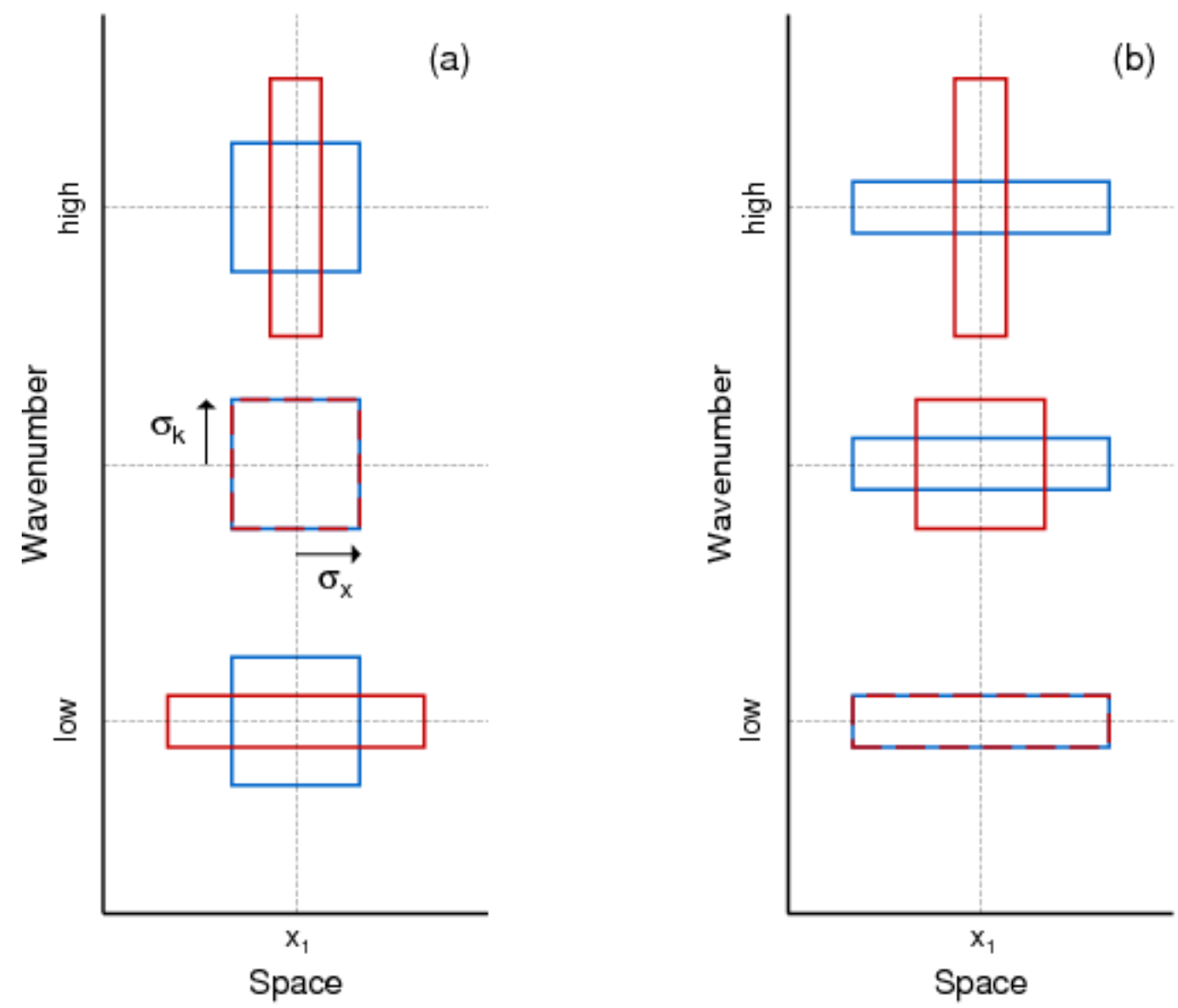

Fig. 6. Support of the wavelet transform (red) and windowed Fourier transform (WFT) (blue) in the space-wavenumber domain. Each image shows the Heisenberg boxes for wavelet and windowed Fourier 'atoms' at a spatial location $x_{1}$, and at three different resolving wavenumbers (low, medium and high). The space and wavenumber resolutions/precisions are given by the half-width of the boxes, $\sigma_{x}$ and $\sigma_{k}$, respectively, which must obey the uncertainty relation $\sigma_{x} \sigma_{k} \geq 2 \pi$. The wavelet atoms in (a) and (b) are the same, but the WFT atoms are different. Wavelets always have a good wavenumber-resolution at low wavenumbers (large-scale wavelets), but a poor one at high wavenumbers (small-scale wavelets), and the resolution is fine-tuned by a single parameter. In contrast the WFT's resolution is determined by the chosen (and then fixed) window size, as well as a parameter (e.g., NW for multitapers). In (a) the WFT window size is chosen such that the WFT has comparable spatial- and wavenumber-resolution to a medium-scaled wavelet; though since 
WFT atoms always preserve their shape, this means that at low wavenumbers the WFT will have a better spatial-resolution (but a worse wavenumber-resolution) than the wavelet transform, and at high wavenumbers a worse spatial-resolution (but a better wavenumberresolution). In (b) the WFT window size is chosen such that the WFT has equivalent spatialand wavenumber-resolution as a large-scaled wavelet; this means that the WFT will have a worse spatial-resolution (but a better wavenumber-resolution) than the wavelet transform at all higher wavenumbers. 

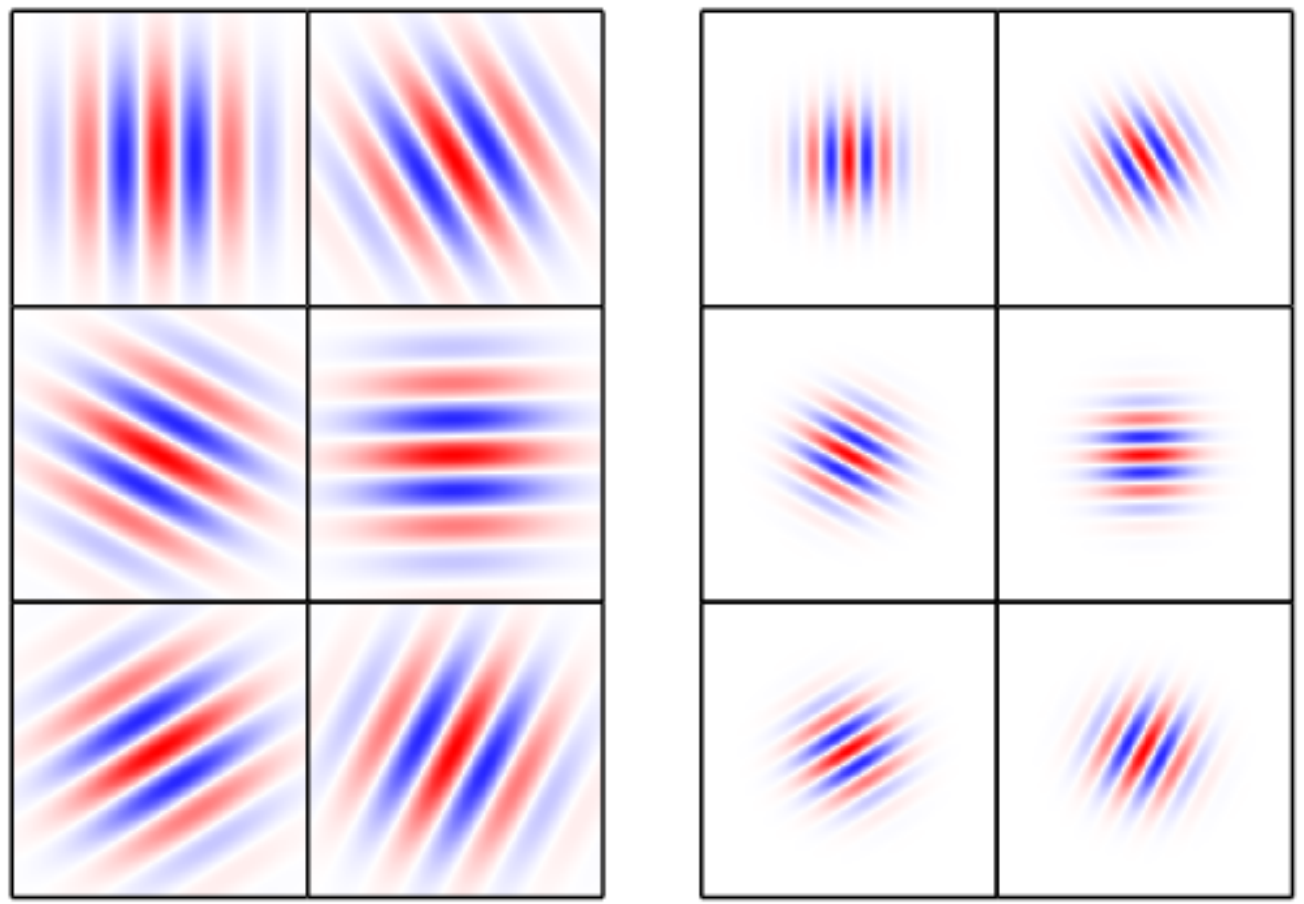

Fig. 7. The real part of 6 2D Morlet wavelets in the space domain at large (left) and small (right) scales, each at a different azimuth (imaginary part not shown). The data are convolved with the wavelets at many scales, and scales are mapped to wavenumber. The convolution yields wavelet coefficients as functions of space, wavenumber and azimuth; their modulussquared gives the wavelet power spectrum. Red colours are positive, blue negative. 


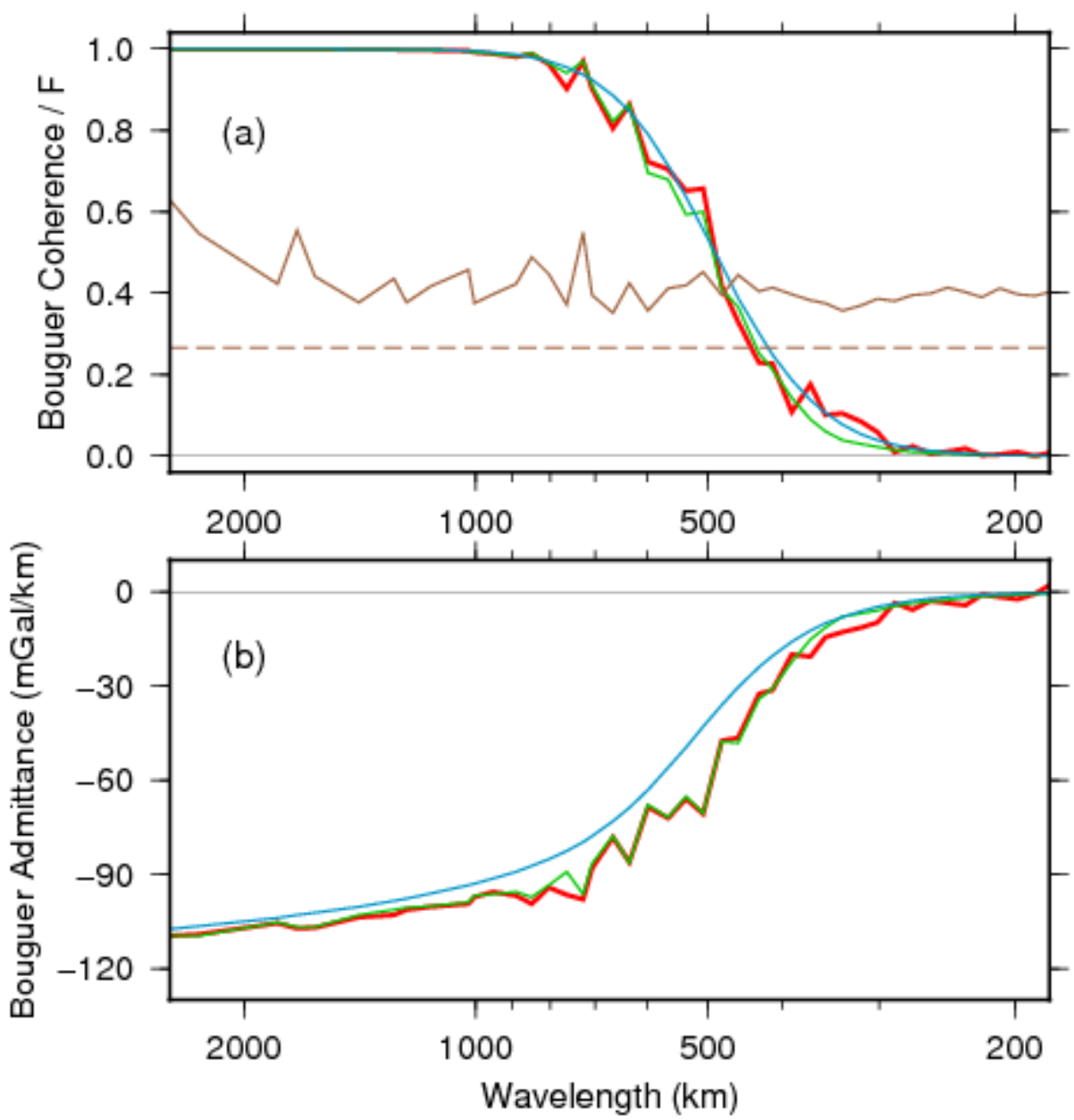

Fig. 8. Results from inversion of an observed Bouguer coherence by load deconvolution and analytic uniform- $f$ inversion. (a) The observed Bouguer coherence (red) and the predicted versions from load deconvolution (green) and uniform- $f$ inversion (blue). The plot also shows the loading ratio, $F=f /(1+f)$, in brown, solid from load deconvolution, dashed from uniform- $f$ inversion. (b) The observed Bouguer admittance for the data (red), and the corresponding predicted admittances given by the two inversion methods. Note that the observed admittance is not inverted, but predicted versions can still be estimated during inversion. In this example the observed and predicted admittance match when load deconvolution inversion of the coherence is used, but not with uniform- $f$ inversion. 


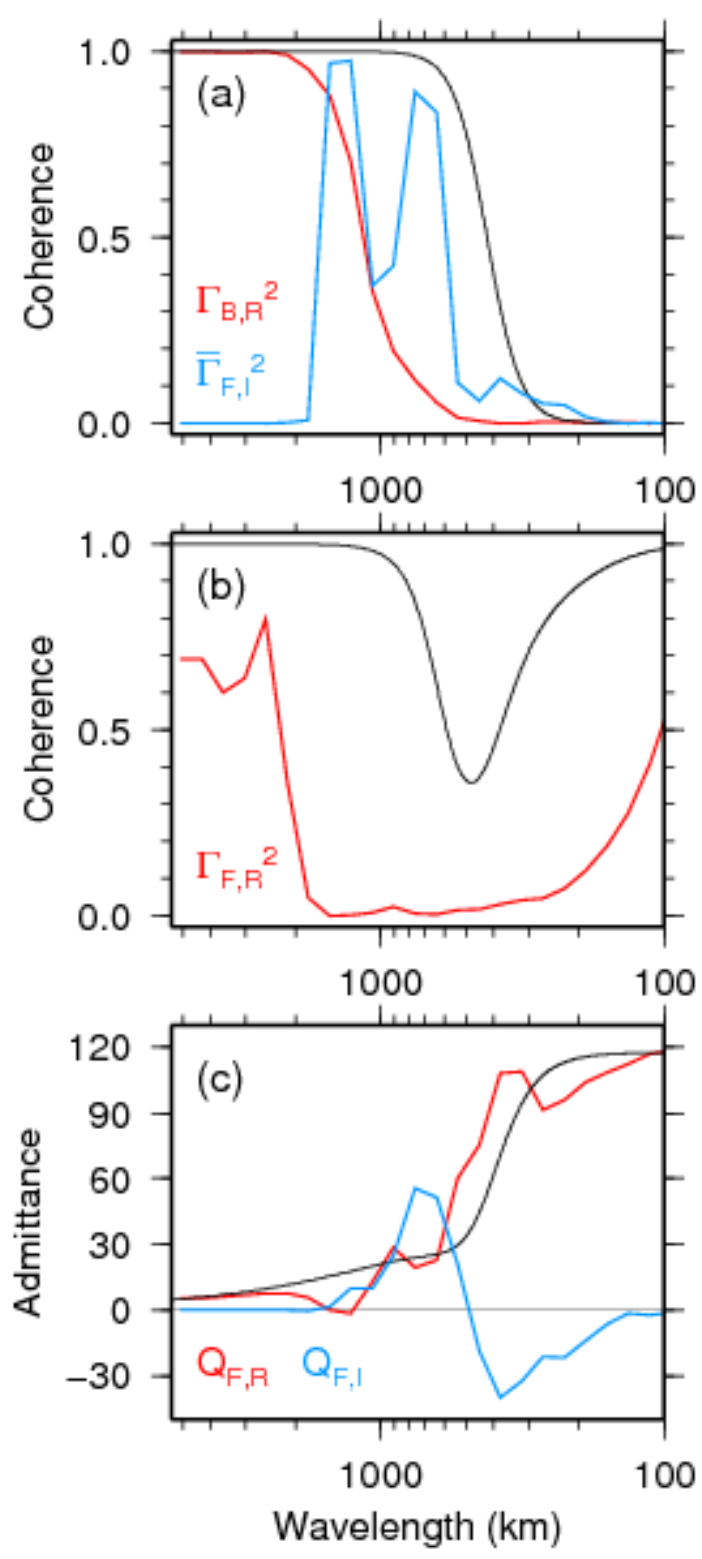

Fig. 9. The effect of noise (blue curves) on the observed (a) Bouguer coherence, (b) free air coherence, and (c) free air admittance (all red curves). Synthetic gravity and topography data were generated by loading on a plate of uniform $T_{e}=40 \mathrm{~km}$ and $f=1$, using the method of Macario et al. (1995). The noise was generated using the methods given in Crosby (2007) and Kirby and Swain (2009) for a plate of the same $T_{e}$, and corresponds to unexpressed loading. The noise was then added to the gravity grids, and the (global) observed Bouguer squared real coherency $\left(\Gamma_{B, R}^{2}\right)$, free air squared real coherency $\left(\Gamma_{F, R}^{2}\right)$, and free air admittance (real 
part, $Q_{F, R}$ ) (the red curves) retrieved using the fan wavelet transform. The theoretical curves for the model are in black. The blue curves show (a) the normalised squared imaginary free air coherency $\left(\bar{\Gamma}_{F, I}^{2}\right)$, and (c) the imaginary admittance $\left(Q_{F, I}\right)$; these are estimates of the noise, and it can be seen how the presence of noise biases the two coherences considerably, while not affecting the admittance very much. See Kirby and Swain (2009) for more information. 

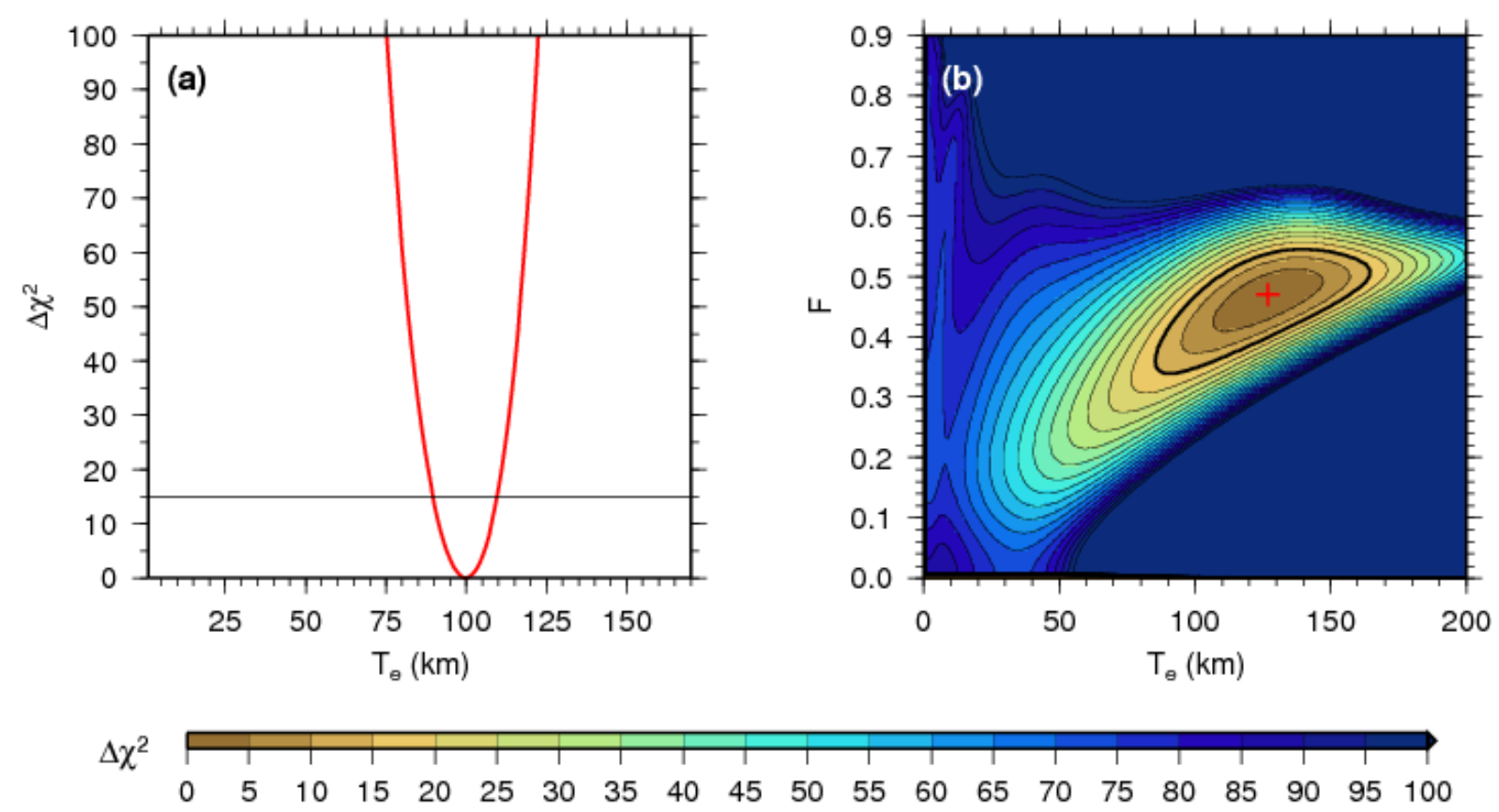

Fig. 10. Misfit (a) curve and (b) surface, showing $\chi^{2}$ values relative to the best-fitting parameter value. (a) The misfit curve from load deconvolution inversion of the Bouguer coherence, where $T_{e}$ is the only parameter solved for. The horizontal black line shows the level of the 99.9\% confidence interval (e.g., Press et al., 1992), which is used to estimate the errors on $T_{e}$. (b) The misfit surface from analytic inversion of the free air admittance, where $T_{e}$ and $F[=f /(1+f)]$ are the parameters solved for. The red cross shows the location of the best-fitting parameter values, while the thicker contour shows the $99.9 \%$ confidence limits, which are used to estimate the errors on $T_{e}$ and $F$. These results are obtained from actual North American data. 